\title{
Cognitive function and cerebral small vessel disease in hypertension
}

Citation for published version (APA):

Uiterwijk, R. (2017). Cognitive function and cerebral small vessel disease in hypertension. [Doctoral Thesis, Maastricht University]. Datawyse / Universitaire Pers Maastricht.

https://doi.org/10.26481/dis.20171207ru

Document status and date:

Published: 01/01/2017

DOI:

10.26481/dis.20171207ru

Document Version:

Publisher's PDF, also known as Version of record

\section{Please check the document version of this publication:}

- A submitted manuscript is the version of the article upon submission and before peer-review. There can be important differences between the submitted version and the official published version of record.

People interested in the research are advised to contact the author for the final version of the publication, or visit the DOI to the publisher's website.

- The final author version and the galley proof are versions of the publication after peer review.

- The final published version features the final layout of the paper including the volume, issue and page numbers.

Link to publication

\footnotetext{
General rights rights.

- You may freely distribute the URL identifying the publication in the public portal. please follow below link for the End User Agreement:

www.umlib.nl/taverne-license

Take down policy

If you believe that this document breaches copyright please contact us at:

repository@maastrichtuniversity.nl

providing details and we will investigate your claim.
}

Copyright and moral rights for the publications made accessible in the public portal are retained by the authors and/or other copyright owners and it is a condition of accessing publications that users recognise and abide by the legal requirements associated with these

- Users may download and print one copy of any publication from the public portal for the purpose of private study or research.

- You may not further distribute the material or use it for any profit-making activity or commercial gain

If the publication is distributed under the terms of Article $25 \mathrm{fa}$ of the Dutch Copyright Act, indicated by the "Taverne" license above, 
COGNITIVE FUNCTION AND CEREBRAL SMALL VESSEL DISEASE IN HYPERTENSION 
(c) R. Uiterwijk, Maastricht 2017

ISBN: 9789461597632

Lay-out en cover design: www.evelienjagtman.com

Print: Datawyse | Universitaire Pers Maastricht 


\section{COGNITIVE FUNCTION AND CEREBRAL \\ SMALL VESSEL DISEASE IN HYPERTENSION}

\section{PROEFSCHRIFT}

ter verkrijging van de graad van doctor aan de Universiteit Maastricht, op gezag van de Rector Magnificus, Prof. dr. Rianne M. Letschert volgens het besluit van het College van Decanen,

in het openbaar te verdedigen

op donderdag 7 december 2017 om 16.00 uur

door

Renske Uiterwijk 


\section{Promotor}

Prof. dr. R.J. van Oostenbrugge

\section{Co-promotor}

Dr. J.E.A. Staals

\section{Beoordelingscommissie}

Prof. dr. R.W.H.M. Ponds (voorzitter)

Prof. dr. A.P. Aldenkamp (Technische Universiteit Eindhoven)

Prof. dr. G.J. Biessels (Universitair Medisch Centrum Utrecht)

Dr. E.G. Gerrits

Dr. W.M. Palm

The research described in this thesis was performed at the Department of Neurology, Maastricht University Medical Centre, and within the School for Mental Health and Neuroscience (MHeNs) and was funded by Stichting de Weijerhorst. 


\section{TABLE OF CONTENTS}

Chapter 1 General introduction 7

Chapter 2 Subjective cognitive failures in patients with hypertension are 23 related to cognitive performance and cerebral microbleeds

Chapter 3 Endothelial activation is associated with cognitive performance in patients with hypertension

Chapter 4 Framingham stroke risk profile is related to cerebral small vessel disease progression and lower cognitive performance in patients with hypertension

Chapter 5 Hypertensive organ damage predicts future cognitive performance: A 9-year follow-up study in patients with hypertension

Chapter 6 Total cerebral small vessel disease MRI score is associated with cognitive decline in executive function in patients with hypertension

Chapter 7 MRI progression of cerebral small vessel disease and cognitive decline in patients with hypertension

Chapter 8 General discussion

Summary

Nederlandse samenvatting

Valorisation

Dankwoord 157

Curriculum Vitae 



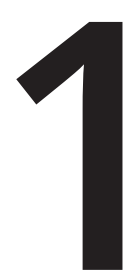

General introduction 



\section{GENERAL INTRODUCTION}

\section{Hypertension}

Hypertension is a highly prevalent condition. In 2000, it was estimated that hypertension affects 972 million adults worldwide and this is predicted to increase by about $60 \%$ to a total of 1.56 billion in 2025. ${ }^{1}$ Hypertension is a leading cause of morbidity and mortality, ${ }^{2}$ because it causes damage in several end organs, such as the kidneys, heart and eyes. ${ }^{3-7}$ Hypertension does not similarly affect all organs. Organ damage can co-occur in several organs, but can also be limited to one. ${ }^{8}$ Little is known about the implications of combined hypertensive organ damages. Guidelines recommend to routinely assess hypertensive organ damage in the heart and kidneys. ${ }^{9}$ Brain damage is less recognized as hypertensive end-organ damage and is an under-researched area, even though the brain is one of the main target organs affected by hypertension. ${ }^{2}$

\section{Cerebral small vessel disease}

Hypertension is, besides age, the most important risk factor for haemorrhagic and ischemic stroke. ${ }^{10}$ About 54\% of strokes worldwide are attributable to hypertension. ${ }^{11}$ In addition, hypertension is strongly associated with cerebral small vessel disease (CSVD). The term cSVD is used to describe all pathological processes that affect the small vessels of the brain, including small arteries, arterioles, capillaries and small veins. ${ }^{12}$ The most prevalent hypertension-related small vessel pathology is arteriolosclerosis, which is also called age- and vascular risk-factor-related cSVD. ${ }^{12}$

The precise pathophysiology of cSVD is unknown, but the occurrence of inflammation and endothelial activation in small perforating vessels, ${ }^{13}$ which also has been suggested in the development of hypertension, ${ }^{14}$ probably plays an important role. Endothelial activation and inflammation lead to an increase in the permeability of the blood-brain barrier and induce microvascular dysfunction. Both leakage of the blood-brain barrier and microvascular dysfunction are thought to play a pivotal role in subsequent CSVD-related brain damage. ${ }^{15,16}$

Unlike large vessels, the small perforating brain vessels are difficult to visualize in vivo. Therefore, the lesions that occur in the brain parenchyma and are thought to be caused by pathological processes in the small vessels have been accepted as markers of CSVD. ${ }^{12}$ These lesions can be seen on neuroimaging, preferably Magnetic Resonance Imaging $(\mathrm{MRI})$, and include white matter hyperintensities (WMH), lacunes, cerebral microbleeds and perivascular spaces (figure 1). 
Figure 1. MRI markers of cerebral small vessel disease

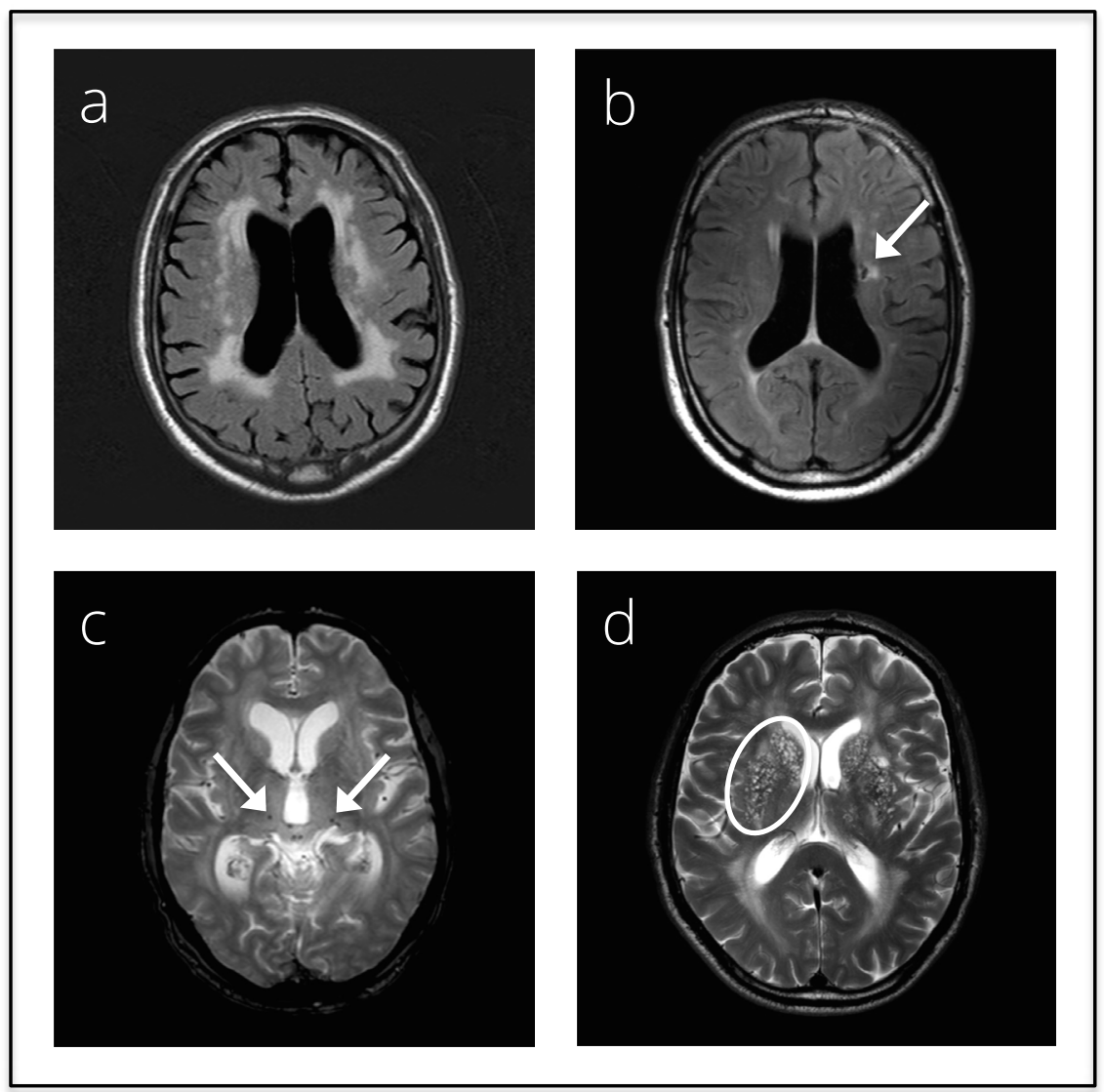

a) white matter hyperintensities, FLAIR image; b) lacune, FLAIR image; c) cerebral microbleeds, T2*-weighted image; d) perivascular spaces, T2-weighted image

WMH are bilateral, mostly symmetrical, areas in the periventricular and deep white matter of the brain and appear as hyperintense signals on T2-weighted and FLAIR MRI. Lacunes are defined as round or ovoid, subcortical, fluid-filled cavities of between 3 and 15 mm diameter and are consistent with a chronic small subcortical infarct in the territory of one perforating arteriole. ${ }^{17}$ Signal intensity is similar to cerebrospinal fluid on all MRI sequences, with generally a surrounding hyperintense rim on FLAIR images. Cerebral microbleeds are small ( $<10 \mathrm{~mm}$ ) hypointense lesions, visible on $\mathrm{T} 2{ }^{*}$-weighted gradient echo or susceptibility-weighted sequences. ${ }^{17}$ They are most commonly found in the cortico-subcortical junction or in the deep grey or white matter in the cerebral hemispheres, brainstem and cerebellum. Perivascular spaces are fluid-filled spaces surrounding the perforating vessels of the brain. On all MRI sequences, they have signal intensity similar to 
that of cerebrospinal fluid. They follow the course of penetrating arteries, so they appear linear when imaged parallel to the course of a vessel, and round or ovoid, with a diameter of $<3 \mathrm{~mm}$, when imaged perpendicular to the course of a vessel. ${ }^{17}$

CSVD can lead to acute clinical symptoms, which manifests itself mainly by lacunar stroke. A lacunar stroke results from a small subcortical infarct due to an occlusion of a single perforating artery. ${ }^{18}$ It is thought to evolve into a lacune over time, although sometimes they disappear on imaging or resemble a white matter hyperintensity. ${ }^{19-21}$ Besides these acute stroke symptoms, cSVD is clinically associated with slowly progressive symptoms, such as gait disorders, ${ }^{22,23}$ unrecognized stroke-like symptoms ${ }^{24}$ and post-stroke depression. ${ }^{25,26}$ In addition, multiple studies have focused on the implications of cSVD on cognitive performance or impairment.

\section{Vascular cognitive impairment}

Vascular cognitive impairment ( $\mathrm{VCl}$ ) indicates the entire range of cognitive deficits associated with and presumed to be caused by cerebrovascular disease. ${ }^{27-29}$ It includes mild cognitive impairment (MCl) and vascular dementia. The latter refers to the most severe cases of $\mathrm{VCl}$, in which the cognitive deficits negatively affect daily functioning. It is the second most common type of cognitive impairment, after Alzheimer's disease. Several subtypes of $\mathrm{VCl}$ can be distinguished, based on the mechanism of cause. ${ }^{29}$ These include multi-infarct, strategic infarct, hemorrhagic, hypoperfusion and mixed dementia. However, the most common subtype is VCl associated with cerebral small vessel disease. ${ }^{29}$ Accordingly, hypertension is a major contributor to vascular cognitive impairment. ${ }^{30}$

\section{cSVD and cognitive function}

Cross-sectional studies on MRI markers of CSVD and cognitive function: studies have linked the presence or degree of the MRI markers of CSVD to lower cognitive performance. These studies have been performed in several different populations, including community-based, stroke and memory clinic populations. Many studies focused on $\mathrm{WMH}^{31-34}$ or lacunes ${ }^{32,34-36}$ and showed associations with global cognitive function and the domains of processing speed and executive function. Evidence regarding cerebral microbleeds and perivascular spaces is less conclusive. Some studies have shown associations between perivascular spaces and information processing speed ${ }^{37}$ and non-verbal reasoning, ${ }^{38}$ but results of the association between degree of perivascular spaces and impairment in several cognitive domains are not consistent. ${ }^{39}$ Regarding cerebral microbleeds, associations between presence and number of cerebral microbleeds and cognition, specifically executive function and information processing speed, have been shown in different populations. ${ }^{40-42}$ 
Longitudinal studies on baseline MRI markers of CSVD and cognitive decline: Fewer studies have performed longitudinal research to MRI markers of cSVD and cognitive decline, of which most investigated WMH or lacunes. In the Rotterdam Scan Study, which is part of the large population-based Rotterdam Study, it was shown that presence of cerebral infarcts at baseline was associated with a doubled risk of dementia and a steeper decline in global cognitive function over a mean of 3.6 years. ${ }^{43}$ Severity of periventricular WMH and cerebral infarcts were associated with a steeper decline in cognitive function over a mean of 5.2 years. ${ }^{44}$ In the 3 -year follow-up of the LADIS study, which included subjects between 65 and 84 years with at least mild WMH, baseline WMH volume predicted cognitive decline, while baseline number of lacunes did not have an independent association with cognitive decline. ${ }^{45} \mathrm{~A}$ study in first-ever lacunar stroke patients showed that severity of $\mathrm{WMH}$ and total number of lacunes were independent predictors of development of $\mathrm{VCl}$ 3 to 5 years after stroke. ${ }^{46}$ Only one longitudinal study could be found investigating the relation between perivascular spaces and cognition, which showed that a higher degree of perivascular spaces was associated with a stronger rate of cognitive decline and with an increase in the risk of incident dementia. ${ }^{47}$ Also regarding cerebral microbleeds, little research investigated cognitive decline, but it is suggested that occurrence of executive impairment during follow-up is more prevalent in patients with cerebral microbleeds. ${ }^{48}$

Most of these studies investigated individual MRI markers of CSVD, while in reality MRI markers of cSVD often co-occur in one patient. A few studies investigated the combined effect of WMH and lacunes on cognitive function or decline, ${ }^{45,49}$ but no study combined all MRI markers. To capture the total CSVD-related brain damage, a total SVD score was proposed, which integrates all individual MRI markers into one measure. ${ }^{50}$ Cross-sectional studies have shown that this total SVD score is associated with higher blood pressure levels, ${ }^{51}$ hypertension, age, other risk factors for SVD ${ }^{50}$ and lower cognitive performance. ${ }^{52,53}$ This SVD score has never been applied in Iongitudinal cognition research. Therefore, it is unclear whether a total score of cSVD-related brain damage, instead of individual MRI markers, is more sensitive to predict future cognitive decline.

Longitudinal studies on progression of MRI markers of CSVD and cognitive decline: insufficient studies have investigated the association between progression of MRI markers of cSVD and cognitive decline over time. A few studies have shown associations between WMH progression and cognitive decline. ${ }^{54-57}$ However, most of these studies did not discriminate between periventricular and subcortical/deep WMH, despite cross-sectional data showing that location of WMH might influence the impact on cognition. ${ }^{31,58,59}$ Regarding the other MRI markers of CSVD and cognitive decline, very few studies have shown associations with new lacunes ${ }^{45,54,60}$ or microbleeds. ${ }^{61}$ In addition, while it is known that baseline presence of CSVD is predictive of cognitive decline, it is unclear whether presence or progression of CSVD is more predictive of cognitive decline. 


\section{Prediction of cognitive performance and CSVD in hypertensive patients}

Already since the 1960s, research described the association between hypertension and cognitive function. ${ }^{62}$ Although the majority of earlier studies used global cognitive outcomes, such as the mini-mental state examination, later research has focused more on specific cognitive domains and their associations with hypertension. Research suggests that hypertension is most strongly associated with deficits in speed of mental processing and executive function. ${ }^{30}$ While impairment in memory function is a characteristic finding for Alzheimer's disease, for which hypertension is a risk factor, there is a lack of consistent evidence for effects of hypertension on memory function.

More insight into other predictors of cognitive performance and MRI markers of CSVD in hypertensive patients is important for identifying high-risk patients. In daily practice, an easily recognizable situation for clinicians may be a hypertensive patient who reports personal experiences of cognitive problems in everyday life, also called subjective cognitive failures. However, positive associations between subjective cognitive failures and objective cognitive impairment, independent of mood and health, were not found in most previous studies. ${ }^{63-66}$ Insight in the implications of subjective cognitive failures for objective cognition and CSVD-related brain damage may show whether or not it is necessary to inquire for the presence of such complaints during consultation of patients with hypertension.

Although scientific evidence is strong about the association between hypertension and CSVD and cognitive performance, the degree of cSVD-related brain damage and cognitive problems greatly differs between hypertensive patients. This means that other factors also influence CSVD and cognitive function, or may moderate the relationship between hypertension, brain damage and cognitive function.

Age is an important factor in both cognitive function ${ }^{67-69}$ and CSVD. ${ }^{33,70,71}$ The effect of age is not the same for all cognitive functions. ${ }^{69}$ Especially in mental processing speed a relatively large effect of age is suggested. ${ }^{72,73}$ While it is often presumed that differences between people increase with age, as in that some people remain cognitively stable and others experience cognitive decline, studies have shown that the individual differences in cognitive performance across age remains relatively stable. ${ }^{69}$

Another important predictor of cognitive function is educational level. It is shown that low education level increases the risk of Alzheimer's dementia ${ }^{74}$ and the risk of experiencing a decline in cognitive function. ${ }^{75}$ In addition, studies have shown that higher level of education may protect against the effect of $\mathrm{WMH}$ on cognitive function ${ }^{76}$ or on the increased risk of $\mathrm{MCl} /$ dementia by $\mathrm{WMH}^{77}$ 
Also other vascular risk factors, besides hypertension, may influence cognition or moderate the relation between hypertension and cognitive function. Diabetes mellitus has been associated with an increased risk of cognitive impairment 78,79 and it may increase the effect of hypertension on the risk of poor cognitive performance. ${ }^{80}$ Smoking has also been associated with poorer cognitive performance ${ }^{81}$ and increased risk of dementia and cognitive decline. ${ }^{82}$ Since vascular risk factors often occur together, a number of vascular risk scores, such as the Framingham stroke risk profile (FSRP), have been developed to incorporate several risk factors into one risk score. The FSRP was originally developed to predict clinical stroke. ${ }^{83,84}$ Even though the FSRP is a prediction score, longitudinal studies examining the association with more silent effects of cerebrovascular disease, such as progression of CSVD and cognitive performance in patients with hypertension are lacking.

\section{Research aim and objectives}

The general aim of this thesis is to determine predictors of cognitive function, cognitive decline and CSVD-related brain damage in patients with hypertension.

This aim is addressed in several research objectives:

- $\quad$ Subjective cognitive failures are frequently mentioned by patients, but in general are given little attention, while these failures might be an indicator of cognitive dysfunction and/or visible brain damage. The first research objective is to examine the association of subjective cognitive failures with objective cognitive performance and with the presence of MRI markers of CSVD in patients with hypertension (chapter 2).

- $\quad$ The second research objective is to study the association between blood markers of endothelial activation and inflammation and cognitive performance three years later. We use a compound score of these blood markers, as this enables the possibility to draw conclusions about the pathogenetic pathways of cognitive problems in hypertensive patients (chapter $\mathbf{3}$ ).

- $\quad$ Since vascular risk factors often occur together, more insight into the use of a composite vascular risk score in hypertensive patients is needed. The third research objective is to examine the association between the Framingham stroke risk profile and cognitive performance and progression of MRI markers of CSVD over 9 years (chapter 4).

- Little is known about the implications of cardiac and renal organ damage independent of brain damage and about the combined presence of hypertensive organ damages for cognitive function. The fourth research objective is to examine if the combined presence of hypertensive organ damage is associated with lower cognitive performance and if cardiac and renal organ damage are predictive of cognitive performance independent of cSVD (chapter 5).

- A total cSVD score might provide a better overview of total cSVD-related brain damage than investigating individual MRI markers. However, as this score has never been 
applied before in longitudinal research, it is unclear if this score is also predictive of future cognitive decline. The fifth research objective is to investigate if the total cSVD score was associated with cognitive decline over 4 years in patients with hypertension (chapter 6).

- Few longitudinal studies have investigated the relation between progression of CSVD and cognitive decline and it is unclear whether the progression or the baseline presence of CSVD is more important for cognitive decline. The sixth research objective is to investigate the association of progression of MRI markers of CSVD with cognitive decline over 4 years and compare this association with baseline presence of the MRI markers (chapter 7).

\section{Study population}

The objectives of chapter 2 to 5 are examined in a cohort of patients with essential hypertension. 212 patients with essential hypertension were included in 2004-2006 in a study on hypertension and brain damage (HYBRiD) and were later invited for follow-up measurements. The timeline of the HYBRiD study, including baseline and follow-up measurements, is shown in figure 2 .

Figure 2. Timeline with components of the study

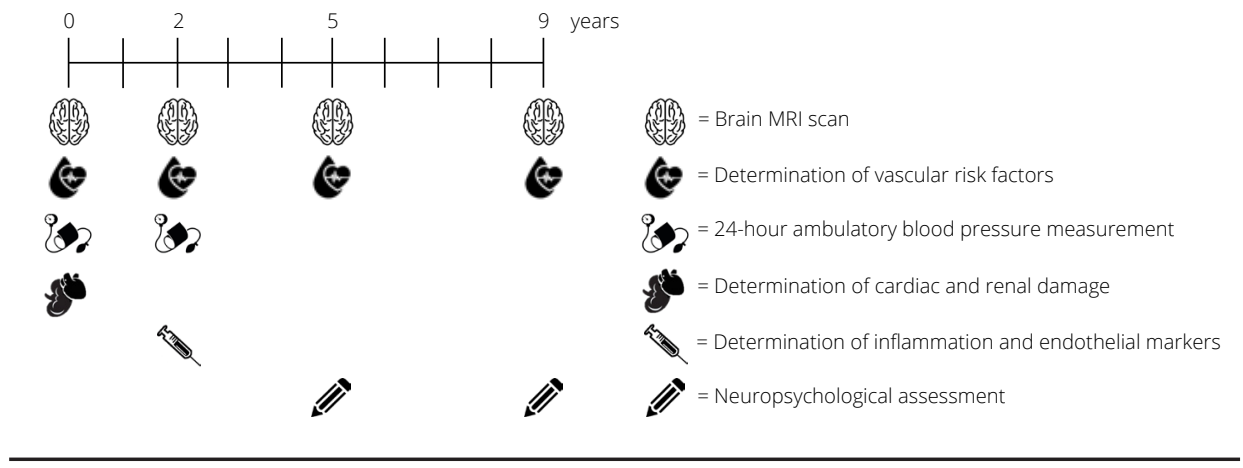

To investigate the research questions of chapter 6 and 7, a patient cohort with a broader spectrum of severity of CSVD was desired. Therefore, in addition to the patients with essential hypertension, we included hypertensive patients with lacunar stroke at the 5-year time point. These patients received identical neuropsychological assessments and MRI scans at year 5 and 9 . 


\section{REFERENCES}

1. Kearney PM, Whelton M, Reynolds K, Muntner P, Whelton PK, He J. Global burden of hypertension: analysis of worldwide data. The Lancet. 2005;365:217-223.

2. Faraco G, ladecola C. Hypertension: A harbinger of stroke and dementia. Hypertension. 2013;62:810-817.

3. Levy D, Anderson MK, Savaga DD, Kannel BW, Christiansen CJ, Castelli PW. Echocardiographically detected left ventricular hypertrophy: Prevalence and risk factors. Ann Intern Med. 1988;108:7-13.

4. Lindroos M, Kupari M, Heikkila J, Tilvis R. Predictors of left ventricular mass in old age: an echocardiographic, clinical and biochemical investigation of a random population sample. Eur Heart J. 1994;15:769-780.

5. Wesson DE. Hypertension and the kidney. Curr Hypertens Rep. 2001;3:511-516.

6. Cerasola G, Mulè G, Cottone S, Nardi E, Cusimano P. Hypertension, microalbuminuria and renal dysfunction: The Renal Dysfunction in Hypertension (REDHY) study. J Nephrol. 2008;21:368-373.

7. Konstantinidis L, Guex-Crosier Y. Hypertension and the eye. Curr Opin Ophthalmol. 2016;27:514-521.

8. Henskens LH, van Oostenbrugge RJ, Kroon AA, Hofman PA, Lodder J, de Leeuw PW. Detection of silent cerebrovascular disease refines risk stratification of hypertensive patients. J Hypertens. 2009;27:846-853.

9. Greenland P, Alpert JS, Beller GA, Benjamin EJ, Budoff MJ, Fayad ZA, Foster E, et al. 2010 ACCF/AHA guideline for assessment of cardiovascular risk in asymptomatic adults: A report of the American College of Cardiology Foundation/American Heart Association task force on practice guidelines. J Am Coll Cardiol. 2010;56:e50-e103.

10. Veglio F, Paglieri C, Rabbia F, Bisbocci D, Bergui M, Cerrato P. Hypertension and cerebrovascular damage. Atherosclerosis. 2009;205:331-341.

11. Lawes CM, vander Hoorn S, Rodgers A. Global burden of blood-pressure-related disease, 2001. The Lancet. 2008;371:1513-1518.

12. Pantoni L. Cerebral small vessel disease: from pathogenesis and clinical characteristics to therapeutic challenges. Lancet Neurol. 2010;9:689-701.

13. Wardlaw JM, Smith C, Dichgans M. Mechanisms of sporadic cerebral small vessel disease: insights from neuroimaging. Lancet Neurol. 2013;12:483-497.

14. Brandes RP. Endothelial dysfunction and hypertension. Hypertension. 2014;64:924-928.

15. Iadecola C. Hypertension and dementia. Hypertension. 2014;64:3-5.

16. Wardlaw JM. Blood-brain barrier and cerebral small vessel disease. J Neurol Sci. 2010;299:66-71.

17. Wardlaw JM, Smith EE, Biessels GJ, Cordonnier C, Fazekas F, Frayne R, Lindley Rl, et al. Neuroimaging standards for research into small vessel disease and its contribution to ageing and neurodegeneration. Lancet Neurol. 2013;12:822-838.

18. Norrving B. Long-term prognosis after lacunar infarction. Lancet Neurol. 2003;2:238-245.

19. Potter GM, Doubal FN, Jackson CA, Chappell FM, Sudlow CL, Dennis MS, Wardlaw JM. Counting cavitating lacunes underestimates the burden of lacunar infarction. Stroke. 2010;41:267-272.

20. Moreau F, Patel S, Lauzon ML, McCreary CR, Goyal M, Frayne R, Demchuk AM, et al. Cavitation after acute symptomatic lacunar stroke depends on time, location, and MRI sequence. Stroke. 2012;43:1837-1842.

21. Loos CM, Staals J, Wardlaw JM, van Oostenbrugge RJ. Cavitation of deep lacunar infarcts in patients with firstever lacunar stroke: A 2-year follow-up study with MR. Stroke. 2012;43:2245-2247. 
22. De Laat KF, Tuladhar AM, van Norden AG, Norris DG, Zwiers MP, de Leeuw F-E. Loss of white matter integrity is associated with gait disorders in cerebral small vessel disease. Brain. 2011;134:73-83.

23. Choi P, Ren M, Phan TG, Callisaya M, Ly JV, Beare R, Chong W, et al. Silent infarcts and cerebral microbleeds modify the associations of white matter lesions with gait and postural stability. Stroke. 2012;43:1505-1510.

24. Windham BG, Griswold ME, Shibata D, Penman A, Catellier DJ, Mosley TH. Covert neurological symptoms associated with silent infarcts from midlife to older age. Stroke. 2012;43:1218-1223.

25. Santos M, Gold G, Kövari E, Herrmann FR, Bozikas VP, Bouras C, Giannakopoulos P. Differential impact of lacunes and microvascular lesions on poststroke depression. Stroke. 2009;40:3557-3562.

26. Tang WK, Chen Y, Liang H, Chu WCW, Mok VCT, Ungvari GS, Wong KS. Cerebral microbleeds as a predictor of 1-year outcome of poststroke depression. Stroke. 2014;45:77-81.

27. O'Brien JT, Erkinjuntti T, Reisberg B, Roman G, Sawada T, Pantoni L, Bowler JV, et al. Vascular cognitive impairment. Lancet Neurol. 2003;2:89-98.

28. Gorelick PB, Scuteri A, Black SE, DeCarli C, Greenberg SM, ladecola C, Launer LJ, et al. Vascular contributions to cognitive impairment and dementia. Stroke. 2011;42:2672-2713.

29. Dichgans M, Leys D. Vascular cognitive impairment. Circ Res. 2017;120:573-591.

30. Iadecola C, Yaffe K, Biller J, Bratzke LC, Faraci FM, Gorelick PB, Gulati M, et al. Impact of hypertension on cognitive function: A scientific statement from the American Heart Association. Hypertension. 2016;68:e67-e94.

31. De GrootJC, de Leeuw F-E, Oudkerk M, van Gijn J, Hofman A, Jolles J, Breteler MM. Cerebral white matter lesions and cognitive function: the Rotterdam Scan Study. Ann Neurol. 2000;47:145-151.

32. Van der Flier WM, van Straaten EC, Barkhof F, Verdelho A, Madureira S, Pantoni L, Inzitari D, et al. Small vessel disease and general cognitive function in nondisabled elderly: The LADIS study. Stroke. 2005;36:2116-2120.

33. Pantoni L, Poggesi A, Inzitari D. The relation between white-matter lesions and cognition. Curr Opin Neurol. 2007;20:390-397.

34. Geerlings MI, Appelman AP, Vincken KL, Mali WP. Association of white matter lesions and lacunar infarcts with executive functioning: The SMART-MR Study. Am J Epidemiol. 2009;170:1147-1155.

35. Benjamin P, Lawrence AJ, Lambert C, Patel B, Chung AW, MacKinnon AD, Morris RG, et al. Strategic lacunes and their relationship to cognitive impairment in cerebral small vessel disease. Neuroimage Clin. 2014;4:828-837.

36. Carey CL, Kramer JH, Josephson SA, Mungas D, Reed BR, Schuff N, Weiner MW, et al. Subcortical lacunes are associated with executive dysfunction in cognitively normal elderly. Stroke. 2008;39:397-402.

37. Huijts M, Duits A, Staals J, Kroon AA, de Leeuw PW, van Oostenbrugge RJ. Basal ganglia enlarged perivascular spaces are linked to cognitive function in patients with cerebral small vessel disease. Curr Neurovasc Res. 2014;11:136-141.

38. MacLullich AMJ, Wardlaw JM, Ferguson KJ, Starr JM, Seckl JR, Deary IJ. Enlarged perivascular spaces are associated with cognitive function in healthy elderly men. J Neurol Neurosurg Psychiatry. 2004;75:1519-1523.

39. Hurford R, Charidimou A, Fox Z, Cipolotti L, Jager R, Werring DJ. MRI-visible perivascular spaces: relationship to cognition and small vessel disease MRI markers in ischaemic stroke and TIA.J Neurol Neurosurg Psychiatry. 2014;85:522-525.

40. Charidimou A, Werring DJ. Cerebral microbleeds and cognition in cerebrovascular disease: an update.J Neurol Sci. 2012;322:50-55. 
41. Poels MM, Ikram MA, van der Lugt A, Hofman A, Niessen WJ, Krestin GP, Breteler MM, et al. Cerebral microbleeds are associated with worse cognitive function: The Rotterdam Scan Study. Neurology. 2012;78:326-333.

42. Werring DJ, Frazer DW, Coward LJ, Losseff NA, Watt H, Cipolotti L, Brown MM, et al. Cognitive dysfunction in patients with cerebral microbleeds on T2*-weighted gradient-echo MRI. Brain. 2004;127:2265-2275.

43. Vermeer SE, Prins ND, den Heijer T, Hofman A, Koudstaal PJ, Breteler MM. Silent brain infarcts and the risk of dementia and cognitive decline. N Eng/J Med. 2003;348:1215-1222.

44. Prins ND, van Dijk EJ, den Heijer T, Vermeer SE, Jolles J, Koudstaal PJ, Hofman A, et al. Cerebral small-vessel disease and decline in information processing speed, executive function and memory. Brain. 2005;128:20342041.

45. Jokinen H, Gouw A, Madureira S, Ylikoski R, van Straaten E, van Der Flier W, Barkhof F, et al. Incident lacunes influence cognitive decline: The LADIS study. Neurology. 2011;76:1872-1878.

46. Pavlovic AM, Pekmezovic T, Tomic G, Trajkovic JZ, Sternic N. Baseline predictors of cognitive decline in patients with cerebral small vessel disease. J Alzheimers Dis. 2014;42:S37-S43.

47. Zhu Y-C, Dufouil C, Soumaré A, Mazoyer B, Chabriat H, Tzourio C. High degree of dilated Virchow-Robin spaces on MRI is associated with increased risk of dementia. J Alzheimers Dis. 2010;22:663-672.

48. Gregoire S, Smith K, Jäger H, Benjamin M, Kallis C, Brown M, Cipolotti L, et al. Cerebral microbleeds and longterm cognitive outcome: Longitudinal cohort study of stroke clinic patients. Cerebrovasc Dis. 2012;33:430-435.

49. Baune BT, Roesler A, Knecht S, Berger K. Single and combined effects of cerebral white matter lesions and lacunar infarctions on cognitive function in an elderly population. J Gerontol A Biol Sci Med Sci. 2009;64A:118124.

50. Staals J, Makin SD, Doubal FN, Dennis MS, Wardlaw JM. Stroke subtype, vascular risk factors, and total MRI brain small-vessel disease burden. Neurology. 2014;83:1228-1234.

51. Klarenbeek P, van Oostenbrugge RJ, Rouhl RP, Knottnerus IL, Staals J. Ambulatory blood pressure in patients with lacunar stroke: Association with total MRI burden of cerebral small vessel disease. Stroke. 2013;44:29952999.

52. Huijts M, Duits A, van Oostenbrugge RJ, Kroon AA, de Leeuw PW, Staals J. Accumulation of MRI markers of cerebral small vessel disease is associated with decreased cognitive function: A study in first-ever lacunar stroke and hypertensive patients. Front Aging Neurosci. 2013;5:72.

53. Staals J, Booth T, Morris Z, Bastin ME, Gow AJ, Corley J, Redmond P, et al. Total MRI load of cerebral small vessel disease and cognitive ability in older people. Neurobiol Aging. 2015;36:2806-2811.

54. Van Dijk EJ, Prins ND, Vrooman HA, Hofman A, Koudstaal PJ, Breteler MM. Progression of cerebral small vessel disease in relation to risk factors and cognitive consequences: Rotterdam Scan Study. Stroke. 2008;39:2712 2719 .

55. Van den Heuvel D, ten Dam V, de Craen A, Admiraal-Behloul F, Olofsen H, Bollen E, Jolles J, et al. Increase in periventricular white matter hyperintensities parallels decline in mental processing speed in a non-demented elderly population. J Neurol Neurosurg Psychiatry. 2006;77:149-153.

56. Longstreth W, Arnold AM, Beauchamp NJ, Manolio TA, Lefkowitz D, Jungreis C, Hirsch CH, et al. Incidence, manifestations, and predictors of worsening white matter on serial cranial magnetic resonance imaging in the elderly: The Cardiovascular Health Study. Stroke. 2005;36:56-61. 
57. Schmidt R, Ropele S, Enzinger C, Petrovic K, Smith S, Schmidt H, Matthews PM, et al. White matter lesion progression, brain atrophy, and cognitive decline: The Austrian Stroke Prevention Study. Ann Neurol. 2005;58:610-616

58. Yoon B, Shim YS, Cheong H-K, Hong Y-J, Lee K-S, Park KH, Ahn KJ, et al. White matter hyperintensities in mild cognitive impairment: clinical impact of location and interaction with lacunes and medial temporal atrophy. J Stroke Cerebrovasc Dis. 2014;23:e365-e372.

59. Soriano-Raya J, Miralbell J, López-Cancio E, Bargalló N, Arenillas JF, Barrios M, Cáceres C, et al. Deep versus periventricular white matter lesions and cognitive function in a community sample of middle-aged participants. J Int Neuropsychol Soc. 2012;18:874-885.

60. Longstreth W, Dulberg C, Manolio TA, Lewis MR, Beauchamp NJ, O'Leary D, Carr J, et al. Incidence, manifestations, and predictors of brain infarcts defined by serial cranial magnetic resonance imaging in the elderly: The Cardiovascular Health Study. Stroke. 2002;33:2376-2382.

61. Liem M, Oberstein SL, Haan J, van der Neut I, Ferrari M, van Buchem M, Middelkoop H, et al. MRI correlates of cognitive decline in CADASIL: A 7-year follow-up study. Neurology. 2009;72:143-148.

62. Elias MF, Goodell AL, Dore GA. Hypertension and cognitive functioning: A perspective in historical context. Hypertension. 2012;60:260-268.

63. Mascherek A, Zimprich D, Rupprecht R, Lang FR. What do cognitive complaints in a sample of memory clinic outpatients reflect? GeroPsych. 2011;24:187-195.

64. Jorm AF, Butterworth P, Anstey KJ, Christensen H, Easteal S, Maller J, Mather KA, et al. Memory complaints in a community sample aged 60-64 years: associations with cognitive functioning, psychiatric symptoms, medical conditions, APOE genotype, hippocampus and amygdala volumes, and white-matter hyperintensities. Psychol Med. 2004;34:1495-1506.

65. Duits A, Munnecom T, van Heugten C, van Oostenbrugge RJ. Cognitive complaints in the early phase after stroke are not indicative of cognitive impairment. J Neurol Neurosurg Psychiatry. 2008;79:143-146.

66. Xiong Y-Y, Wong A, Mok VC, Tang W-K, Lam WW, Kwok TC, Chu WC, et al. Frequency and predictors of proxy-confirmed post-stroke cognitive complaints in lacunar stroke patients without major depression. Int J Geriatr Psychiatry. 2011;26:1144-1151.

67. Jolles J, Houx PJ, van Boxtel MPJ, Ponds RWHM. The Maastricht Aging Study: Determinants of cognitive aging. Maastricht: Neuropsych Publishers; 1995.

68. Birren JE, Schaie KW. Handbook of the psychology of aging. Gulf professional publishing; 2001.

69. Salthouse TA. Selective review of cognitive aging. J Int Neuropsycho/ Soc. 2010;16:754-760.

70. Basile AM, Pantoni L, Pracucci G, Asplund K, Chabriat H, Erkinjuntti T, Fazekas F, et al. Age, hypertension, and lacunar stroke are the major determinants of the severity of age-related white matter changes. Cerebrovasc Dis. 2006;21:315-322.

71. Grueter BE, Schulz UG. Age-related cerebral white matter disease (leukoaraiosis): a review. Postgrad Med J. 2012;88:79-87.

72. Salthouse TA. The processing-speed theory of adult age differences in cognition. Psychol Rev. 1996;103:403428.

73. Finkel D, Reynolds CA, McArdle JJ, Pedersen NL. Age changes in processing speed as a leading indicator of 
cognitive aging. Psychol Aging. 2007;22:558

74. Stern Y, Gurland B, Tatemichi TK, Tang MX, Wilder D, Mayeux R. Influence of education and occupation on the incidence of Alzheimer's disease. JAMA. 1994;271:1004-1010.

75. Yaffe K, Fiocco AJ, Lindquist K, Vittinghoff E, Simonsick EM, Newman AB, Satterfield S, et al. Predictors of maintaining cognitive function in older adults: The Health ABC Study. Neurology. 2009;72:2029-2035.

76. Dufouil C, Alperovitch A, Tzourio C. Influence of education on the relationship between white matter lesions and cognition. Neurology. 2003;60:831-836.

77. Mortamais M, Portet F, Brickman AM, Provenzano FA, Muraskin J, Akbaraly TN, Berr C, et al. Education modulates the impact of white matter lesions on the risk of mild cognitive impairment and dementia. Am J Geriatr Psychiatry. 2014;22:1336-1345.

78. Nash DT, Fillit H. Cardiovascular disease risk factors and cognitive impairment. Am J Cardiol. 2006;97:12621265.

79. Baumgart M, Snyder HM, Carrillo MC, Fazio S, Kim H, Johns H. Summary of the evidence on modifiable risk factors for cognitive decline and dementia: A population-based perspective. Alzheimers Dement. 2015;11:718726.

80. Elias PK, Elias MF, D’Agostino RB, Cupples LA, Wilson PW, Silbershatz H, Wolf PA. NIDDM and blood pressure as risk factors for poor cognitive performance: The Framingham Study. Diabetes care. 1997;20:1388-1395.

81. North T-L, Palmer TM, Lewis SJ, Cooper R, Power C, Pattie A, Starr JM, et al. Effect of smoking on physical and cognitive capability in later life: a multicohort study using observational and genetic approaches. BMJ open. 2015;5:e008393.

82. Anstey KJ, von Sanden C, Salim A, O'Kearney R. Smoking as a risk factor for dementia and cognitive decline: A meta-analysis of prospective studies. Am J Epidemiol. 2007;166:367-378.

83. D'Agostino RB, Wolf PA, Belanger AJ, Kannel WB. Stroke risk profile: adjustment for antihypertensive medication. The Framingham Study. Stroke. 1994;25:40-43.

84. Wolf PA, D'Agostino RB, Belanger AJ, Kannel WB. Probability of stroke: a risk profile from the Framingham Study. Stroke. 1991;22:312-318. 




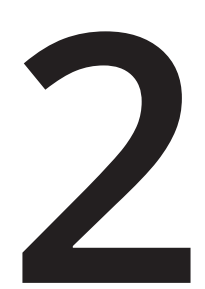

\section{Subjective cognitive failures in patients with hypertension are related to cognitive performance and cerebral microbleeds}

Uiterwijk R, Huijts M, Staals J, Duits A, Gronenschild E, Kroon AA, de Leeuw PW, van Oostenbrugge RJ 


\begin{abstract}
Objectives Previous studies on the relationship between subjective cognitive failures (SCF) and objective cognitive function have shown inconsistent results. In addition, research on the association between SCF and imaging markers of cerebral Small Vessel Disease (CSVD) is limited. We investigated if SCF in essential hypertensive patients, who are at high risk of CSVD, are associated with objective cognitive function and/or MRI manifestations of CSVD.
\end{abstract}

Methods We included 109 hypertensive patients, who underwent extensive neuropsychological assessment, including questionnaires measuring SCF and symptoms of anxiety and depression. Brain MRI was performed to rate the presence of lacunes, cerebral microbleeds, and perivascular spaces, as well as white matter hyperintensities volume.

Results Results showed significant associations between SCF and objectively measured overall cognition ( $B=-0.02 ; 95 \% C l=-0.03$ to -0.005$)$, memory $(B=0.02 ; 95 \% C l=-0.03$ to -0.004), and information processing speed ( $B=-0.02 ; 95 \% \mathrm{Cl}=-0.03$ to -0.001$)$ after adjustment for patient characteristics and vascular risk factors. In addition, SCF were associated with the presence of cerebral microbleeds $(O R=1.12 ; 95 \% \mathrm{Cl}=1.02$ to 1.23) after adjustment for patient characteristics and vascular risk factors, but not with other imaging markers of cSVD.

Conclusion Our study demonstrates that attention for SCF in hypertensive patients is needed, as these may point to lower objective cognitive function what might be due to the presence of cerebral microbleeds. Accordingly, this study emphasizes that neuropsychological assessment and brain imaging need to be considered when hypertensive patients report SCF. 


\section{INTRODUCTION}

Subjective cognitive failures (SCF) are a common feature in elderly people with cardiovascular disease. ' SCF are personal experiences of cognitive problems in everyday life, such as difficulties in memory (e.g. forgetfulness), information processing speed (e.g. slowness in performing simple tasks) and executive function (e.g. problems in planning or reasoning). Although these subjective problems are common, it is not completely clear what they represent. The relationship with objective cognitive performance on neuropsychological assessment is unclear. Although most studies showed no relation with objective cognitive impairment when corrected for mood and health, ${ }^{1-6}$ some studies reported positive associations. 7,8 However, studies have shown a strong association with poor physical health ${ }^{6}$ and depression., 2,6

Research on SCF in patients with hypertension is scarce, despite the increasing support for an association between hypertension and objective cognitive impairment, ${ }^{9-11}$ which might be due to brain damage. Hypertension is a major risk factor for white matter hyperintensities $(\mathrm{WMH})^{12}$ and other MRI manifestations of cerebral Small Vessel Disease (CSVD), ${ }^{13}$ which include lacunes, cerebral microbleeds and perivascular spaces..$^{14} \mathrm{~A}$ possible association between SCF and CSVD-related brain damage, as proposed by previous research, 1,15,16 and objective cognitive impairment has not been examined before in hypertensive patients.

A hypertensive patient expressing SCF may be a recognizable situation for clinicians. To gain more knowledge about the implications of SCF in essential hypertensive patients, this study investigated if SCF are associated with objective cognitive impairment, independent of confounding factors, such as age, sex, symptoms of anxiety and depression, and vascular risk factors. In addition, we examined if SCF are predictive for the presence of MRI markers of CSVD.

\section{METHODS}

\section{Study population}

Originally, 218 patients were recruited from the hypertension outpatient clinic of the Department of Internal Medicine of Maastricht University Medical Centre, the Netherlands, for a study on brain damage in essential hypertensive patients (HYBRiD). ${ }^{17}$ Hypertension was defined as an off-medication, clinically measured conventional blood pressure $\geq 140$ $\mathrm{mm}$ Hg systolic or $\geq 90 \mathrm{~mm} \mathrm{Hg}$ diastolic, or both. Details have been described before. ${ }^{17}$ Patients who agreed to be contacted for follow-up, were offered a brain MRI and cognitive assessment 5 years later, which constitutes the present (cross-sectional) study sample. 
Exclusion criteria were a history of symptomatic cardio- or cerebrovascular disease or contraindications for MRI. Age, gender, body mass index (BMI), smoking, occupational status (currently working or not working (retired, unemployed or on sick leave)), level of education (low, average or high), and the presence of diabetes mellitus or hypercholesterolemia were recorded. At baseline, two 24-hour ambulatory blood pressure monitorings (ABPM) were performed; details about the ABPM have been described before. ${ }^{17}$ Hypertension load was defined as known duration of hypertension (months) * the mean of the two baseline 24-hour mean arterial pressure values. This study was approved by the Medical Ethics Committee of the Maastricht University Medical Centre and all participants gave written informed consent.

\section{Cognitive assessment}

Subjective cognitive failures were assessed with the Cognitive Failure Questionnaire (CFQ). ${ }^{18}$ This questionnaire contains 25 questions about the frequency of failures in perception, memory, and motor function. Total scores range between 0 and 100, with higher scores indicating more subjective cognitive failures. The scale has a high internal consistency in our sample (Cronbach's Alpha=0.88).

Objective cognitive performance was measured with an extensive neuropsychological assessment that covered three main cognitive domains. The memory domain was measured with the Rey Auditory Verbal Learning Test ${ }^{19}$ (RAVLT; immediate recall, delayed recall and delayed recognition), and the Digit Span Forward (subtest of Wechsler Adult Intelligence Scale (WAIS) $\|^{20}{ }^{20}$. The executive function domain was measured with the Stroop Colour Word Test ${ }^{21}$ (SCWT) interference score (time of part 3 minus mean time of parts 1 and 2), Trail Making Test 22 (TMT) interference score (time of part 2 minus time of part 1), Category (animals and professions) ${ }^{23}$ and Letter Fluency, ${ }^{24}$ Letter-Number Sequencing (subtest of WAIS-III) and Digit Span Backward (subtest of WAIS-III). Information processing speed was measured with the Symbol Substitution - Coding (subtest of WAIS-III), TMT part A and SCWT parts 1 and 2. For each patient, compound scores of each domain were calculated by averaging the z-scores of all tests within that domain. In addition, an overall cognition compound score was calculated by averaging the three domain compound scores. The Rotterdam - Cambridge Cognitive Examination (R-CAMCOG) was used to determine the presence of possible dementia, defined as a score $<34 .{ }^{25}$

Symptoms of depression and anxiety were measured using the Hospital Anxiety and Depression Scale (HADS). This questionnaire consists of 7 items regarding depression (total score 0 - 21) and 7 items regarding anxiety (total score 0 - 21). Since symptoms possibly caused by physical problems (e.g., insomnia or weight loss) are not included in the HADS, the scale is considered to be suitable to use in somatic populations. ${ }^{26}$ 


\section{MRI data}

On brain MRI scans (standard axial T2-weighted, FLAIR and T2* gradient echo sequences, Intera 1.5-T, Philips Medical Systems, Best, The Netherlands), two experienced vascular neurologists (JS and RvO) individually rated the presence of lacunes, cerebral microbleeds and perivascular spaces. In cases of disagreement, lesions were ascertained by consensus. Lacunes were identified as sharply demarcated hyperintense lesions ( $<20 \mathrm{~mm}$ ) on T2-weighted images with corresponding hypointense lesions with a hyperintense rim on FLAIR. Cerebral microbleeds were defined as punctate $(<10 \mathrm{~mm})$ homogeneous foci of low signal intensity on T2*-weighted images. Symmetrical hypointensities in the globi pallidi were disregarded, since these most likely represent calcification. Presence of lacunes or cerebral microbleeds was indicated if one or more lacune(s) or cerebral microbleed(s) was present. Perivascular spaces were defined as round, oval or linear shaped lesions with a smooth margin, absence of mass effect and with signal intensity equal to cerebrospinal fluid on T2-weighted imaging and (if visible) hypointense on FLAIR images without hyperintense rim to distinguish them from lacunes. Perivascular spaces were distinguished in two different areas: the basal ganglia and the white matter of the centrum semiovale. In both areas, only the most affected hemisphere was rated on one slice only, using a semi-quantitative scale (mild-moderate-extensive). ${ }^{27}$ Presence of perivascular spaces was indicated if moderate or extensive perivascular spaces were present in that area. WMH were defined as hyperintense areas in the periventricular and deep white matter on both T2-weighted and FLAIR images. WMH volumes were semi-automatically assessed using the image-processing software package GIANT (EG), version 2V1.26 (General Imaging and Analysis Tools; Department of Psychiatry and Neuropsychology, Maastricht University, Maastricht, The Netherlands). ${ }^{28}$ A trained observer ( $\mathrm{MH}$ ) performed the quantitative assessments after reaching excellent interobserver agreement with an experienced neuroradiologist (intraclass correlation of 0.99 for total WMH).

\section{Statistical analysis}

The associations between CFQ score and patient characteristics and vascular risk factors were tested with independent samples t-tests (for categorical variables with two categories), one-way ANOVA (for categorical variables with three categories), Pearson's correlations (for normally distributed continuous variables) or Spearman's correlations (for non-normally distributed continuous variables). The relationships between total CFQ score and the overall cognition score and the scores of the three separate cognitive domains were investigated using simple linear regression analyses. The relationships were then adjusted for patient characteristics (age, gender, occupational status, high or low educational level, anxiety score and depression score) with multiple linear regression analyses (entry method). Next, vascular risk factors (hypertension load, hypercholesterolemia, smoking, diabetes mellitus and BMI) were added to these analyses (entry method). 
To investigate the relationship between total CFQ score and the presence of CSVD manifestations on MRI, simple logistic regression analyses were performed with CFQ score as independent and the presence of each CSVD manifestation as dependent variable. These relationships were then adjusted for patient characteristics and for both patient characteristics and vascular risk factors. The association between CFQ score and WMH volume was examined with a Spearman's correlation, because of the non-normal distribution of WMH volume. To adjust this correlation for patient characteristics, a linear regression analysis with the patient characteristics as independent variables and CFQ as dependent variable was performed, standardized residuals were saved, and these residuals were then used in Spearman's correlation analysis. The same procedure was used to adjust for both patient characteristics and vascular risk factors. IBM SPSS Statistics 20 software was used for all analyses. Results were considered significant at $p<0.05$.

\section{RESULTS}

From the original 218 patients, 198 agreed to be contacted for this study and 112 patients completed brain MRI and neuropsychological assessment. Reasons for exclusion were: not interested ( $n=69)$, ischemic stroke $(n=7)$, death $(n=4)$, pre-existent cognitive problems $(n=3)$, or other reasons ( $n=3)$. Two patients were additionally excluded from analyses because of an R-CAMCOG score <34, and one patient was excluded based on a deviant score on the CFQ. Finally, 109 patients were included in the present analyses. The included patients did not differ from the excluded patients $(n=89)$ regarding age $(56.1 \pm 12.1 \mathrm{vs} .58 .7 \pm 13.4$ years, $p=0.17$ ) and sex (male $58.7 \%$ vs. 44.9\%, $p=0.054$ ). Compound scores for memory, information processing speed, executive function and overall cognition were missing for 2, 1, 1 and 3 patients, respectively; therefore, analyses which included these scores were performed with different numbers of subjects. The characteristics of the patients are shown in table 1.

\section{Subjective cognitive failures}

Mean CFQ score was 33.7 (SD=10.3, range=12-63). The CFQ score was positively correlated with age $(r=0.22, p=0.02)$, HADS anxiety score $(r=0.49, p<0.001)$ and HADS depression score $(r=0.32, p=0.001)$. Currently working patients had significant lower CFQ scores compared with patients who were retired, unemployed or on sick leave $\left(t_{107}=2.24, p=0.03\right)$. There was no difference in CFQ score between sexes $\left(t_{107}=1.72, p=0.09\right)$, smokers and non-smokers $\left(t_{106}=1.03, p=0.31\right)$, patients with and without hypercholesterolemia $\left(t_{103}=-\right.$ $0.96, p=0.34)$, with and without diabetes mellitus $\left(t_{107}=-0.25, p=0.80\right)$ or with different educational levels $\left(F_{2,106}=1.44, p=0.24\right)$. There was no correlation between CFQ score and $\mathrm{BMI}(r=0.02, p=0.82)$, and hypertension load $(r=0.06, p=0.54)$. 
Table 1. Patients' characteristics

\begin{tabular}{ll}
\hline Characteristic & All patients $(\boldsymbol{n}=\mathbf{1 0 9})$ \\
\hline Mean age (years, SD) & $56.1(12.1)$ \\
Male sex (\%) & $64(58.7)$ \\
Educational level (\%): & $27(24.8)$ \\
- Low & $41(37.6)$ \\
- Average & $41(37.6)$ \\
- High & $62(56.9)$ \\
Currently working (\%) & $5(3-8)$ \\
Median of HADS anxiety score (IQR) & $2(1-5)$ \\
Median of HADS depression Score (IQR) & $16(14.8)$ \\
Smokers (\%)* & $111.2(12.6)$ \\
Mean MAP (SD) & $43.2(13.8-129.8)$ \\
Median duration of hypertension (months, IQR) & $4299.3(1537.8-15182.8)$ \\
Median hypertension load (IQR) & $2(1.8)$ \\
Diabetes mellitus (\%) & $40(38.1)$ \\
Hypercholesterolemia (\%)* & $27.3(4.2)$ \\
Body Mass Index (SD) & $33.7(10.3)$ \\
Mean CFQ score (SD) &
\end{tabular}

$S D=$ standard deviation; $I Q R=$ interquartile range; HADS=hospital anxiety and depression score; MAP=mean arterial pressure; $\mathrm{CFQ}=$ cognitive failures questionnaire

* 1 missing value for smoking; 4 missing values for hypercholesterolemia

\section{SCF and objective cognitive performance}

In simple linear regression analyses, the CFQ score was significantly associated with overall cognition, memory, executive function and information processing speed (table 2). After adjusting for age, gender, high and low educational level, occupational status, anxiety score and depression score, these associations remained significant. After additional adjustment for vascular risk factors, the associations between overall cognition, memory and information processing speed were still significant (table 2).

\section{SCF and cSVD manifestations on MRI}

The prevalence of CSVD manifestations and the median WMH volume are shown in table 3. In unadjusted analyses, CFQ was not associated with any of the MRI manifestations of cSVD (table 4). However, after adjustment for patient characteristics (age, gender, high and low educational level, occupational status, anxiety score and depression score), the association between CFQ and cerebral microbleeds became significant and this remained after further adjustment for vascular risk factors (table 4). 
Table 2. Association between CFQ and cognition

\begin{tabular}{lllll}
\hline Statistical analysis & $\begin{array}{l}\text { Overall } \\
\text { cognition; } \\
\mathbf{B}(95 \% \mathrm{Cl})\end{array}$ & $\begin{array}{l}\text { Memory; } \\
\mathbf{B}(95 \% \mathrm{Cl})\end{array}$ & $\begin{array}{l}\text { Executive } \\
\text { function; } \\
\mathbf{B}(95 \% \mathrm{Cl})\end{array}$ & $\begin{array}{l}\text { Information } \\
\text { processing } \\
\text { speed; } \mathbf{B}(\mathbf{9 5 \%} \mathrm{Cl})\end{array}$ \\
\hline Unadjusted & -0.02 & -0.02 & -0.02 & -0.02 \\
& $(-0.04$ to -0.01$) \ddagger$ & $(-0.03$ to -0.007$) \S$ & $(-0.03$ to -0.008$) \S$ & $(-0.04$ to -0.01$) \S$ \\
$\begin{array}{l}\text { Adjusted for patient } \\
\text { characteristics* }\end{array}$ & -0.02 & -0.02 & -0.01 & -0.02 \\
$\begin{array}{l}\text { Adjusted for patient } \\
\text { characteristics and vascular } \\
\text { risk factorst }\end{array}$ & $(-0.03$ to -0.007$) \S$ & $(-0.03$ to -0.004$) \|$ & $(-0.03$ to -0.002$) \|$ & $(-0.03$ to -0.001$) \|$ \\
\hline
\end{tabular}

$C F Q=$ cognitive failures questionnaire; $B=$ unstandardized regression coefficient; $C$ =confidence Interval

* Age, educational level, occupational status, gender, anxiety score and depression score

† Hypertension load, smoking, hypercholesterolemia, diabetes mellitus and body mass index

$\ddagger p<0.001$

$\S p<0.01$

$\| p<0.05$

Table 3. Prevalence of cSVD manifestations on MRI

\begin{tabular}{ll}
\hline Presence of cSVD manifestations & All patients $(\boldsymbol{n = 1 0 9 )}$ \\
\hline Lacunes (\%) & $24(22.0)$ \\
Cerebral microbleeds (\%) & $16(14.7)$ \\
Perivascular spaces basal ganglia (\%) & $17(15.6)$ \\
Perivascular spaces centrum semiovale (\%) & $60(55.0)$ \\
Median of WMH volume $\left(\mathrm{mm}^{3}, I Q R\right)$ & $655(146-2012)$ \\
\hline
\end{tabular}

$C S V D=$ cerebral small vessel disease; $W M H=$ white matter hyperintensities; $I Q R=$ interquartile range

Table 4. Association between CFQ and CSVD manifestations

\begin{tabular}{|c|c|c|c|c|c|}
\hline Statistical analysis & $\begin{array}{l}\text { Lacunes; } \\
\text { OR }(95 \% \mathrm{CI})\end{array}$ & $\begin{array}{l}\text { Cerebral } \\
\text { micro- } \\
\text { bleeds; } \\
\text { OR }(95 \% \mathrm{CI})\end{array}$ & $\begin{array}{l}\text { Perivascular } \\
\text { spaces basal } \\
\text { ganglia; } \\
\text { OR }(95 \% \mathrm{Cl})\end{array}$ & $\begin{array}{l}\text { Perivascular } \\
\text { spaces } \\
\text { centrum } \\
\text { semiovale; } \\
\text { OR }(95 \% \mathrm{Cl})\end{array}$ & $\begin{array}{l}\text { WMH; } \\
r(p)\end{array}$ \\
\hline Unadjusted & $\begin{array}{l}1.00 \\
(0.96 \text { to } 1.05)\end{array}$ & $\begin{array}{l}1.02 \\
(0.97 \text { to } 1.07)\end{array}$ & $\begin{array}{l}1.02 \\
(0.97 \text { to } 1.07)\end{array}$ & $\begin{array}{l}1.03 \\
\text { (0.99 to } 1.07)\end{array}$ & $\begin{array}{l}0.17 \\
(0.09)\end{array}$ \\
\hline Adjusted for patient characteristics* & $\begin{array}{l}0.98 \\
(0.92 \text { to } 1.03)\end{array}$ & $\begin{array}{l}1.10 \\
\text { (1.01 to } 1.19) \ddagger\end{array}$ & $\begin{array}{l}1.00 \\
\text { (0.93 to } 1.07)\end{array}$ & $\begin{array}{l}1.03 \\
\text { (0.99 to } 1.09)\end{array}$ & $\begin{array}{l}0.023 \\
(0.81)\end{array}$ \\
\hline $\begin{array}{l}\text { Adjusted for patient characteristics } \\
\text { and vascular risk factors } \dagger\end{array}$ & $\begin{array}{l}0.97 \\
(0.91 \text { to } 1.04)\end{array}$ & $\begin{array}{l}1.12 \\
(1.02 \text { to } 1.23) \ddagger\end{array}$ & $\begin{array}{l}0.99 \\
(0.91 \text { to } 1.07)\end{array}$ & $\begin{array}{l}1.06 \\
(1.00 \text { to } 1.11)\end{array}$ & $\begin{array}{l}0.029 \\
(0.77)\end{array}$ \\
\hline
\end{tabular}

$C F Q=$ cognitive failures questionnaire; $C S V D=$ cerebral small vessel disease; $W M H=$ white matter hyperintensities; $O R=$ odds ratio; $\mathrm{Cl}=$ confidence interval

* Age, educational level, occupational status, gender, anxiety score and depression score

† Hypertension load, smoking, hypercholesterolemia, diabetes mellitus and body mass index

$\neq p<0.05$ 


\section{DISCUSSION}

In the present study, we investigated the association between SCF and objective cognitive function and CSVD manifestations on MRI in 109 hypertensive patients. We demonstrated that SCF were significantly related to objective cognitive function in three important domains, independent of age, gender, occupational status, educational level, and symptoms of anxiety and depression. After additional adjustment for vascular risk factors, overall cognition, memory and information processing speed remained significantly related to SCF. Although in other patient populations, only few studies have reported a relationship between SCF and cognitive performance. ${ }^{78,29}$ In contrast, the majority of studies reported no such association, but found a relationship between SCF and mood ${ }^{3,4}$. This discrepancy might be due to several drawbacks in these studies. Some of these studies based their SCF measure on a small number of questions about cognitive complaints; $2,6,30$ investigated memory complaints only; ${ }^{6}$ or used a short screening instrument ${ }^{1}$ for cognitive functioning, such as the Mini-Mental State Examination (MMSE). In addition, no association between SCF and cognitive performance was found in stroke patients. ${ }^{3,5}$ It is possible that this association is only apparent in healthy people, without a preceding symptomatic event, such as a stroke. This might reduce the occurrence of problems in adjustment, such as anxiety and depression, after an event.

This study showed an association between SCF and the presence of cerebral microbleeds in hypertensive patients, after adjustment for patient characteristics and vascular risk factors. Only one other study also found a relationship between the presence of microbleeds and SCF. ${ }^{31}$ In our study, SCF were not significantly related to the presence of other CSVD manifestations, namely WMH, lacunes and perivascular spaces. Although not studied before in a group of hypertensive patients, this is consistent with most of the earlier results found in other populations.,7,32 One study' investigated the relationship in patients with cardiovascular disease, including hypertensive patients, and showed that SCF were related to WMH. However, that study did not adjust for possible confounders. Two studies found an association between WMH and SCF after adjustment for possible confounders in non-demented elderly from the general population. ${ }^{15,16}$ The contradicting results might be explained by the different sample or by a lack of statistical power in our study, since our sample size was relatively small compared with these studies ( $n=109 \mathrm{vs.}$ $n=1779$ and $n=1049$, respectively).

Strengths of our study include the use of a standardized, 25-item questionnaire to determine SCF, analysis of the SCF score as a continuous variable and the extensive neuropsychological assessment, performed by the same psychologist for all patients. A limitation of the study is the cross-sectional design. Longitudinal studies in hypertensive 
patients are needed to examine the long-term predictive value of subjective cognitive failures on cognitive function and brain damage and to provide more evidence that SCF could be an early predictor of dementia, as has been suggested in other studies involving healthy elderly people. ${ }^{33-35}$

In conclusion, the findings of our study indicate that it is necessary to inquire for the presence of cognitive complaints during consultation of hypertensive patients, since SCF might be a symptom of objective cognitive impairment and cerebral microbleeds. In contrast to other studies, our results show that SCF point to more than anxiety and depression symptoms. As such, extensive neuropsychological assessment and brain imaging should be considered in these patients. More particularly, it is important to identify patients with SCF, since results have shown an association between certain antihypertensive medications and better memory performance in patients with subjective memory complaints. ${ }^{36}$ 


\section{REFERENCES}

1. Haley AP, Hoth KF, Gunstad J, Paul RH, Jefferson AL, Tate DF, Ono M, et al. Subjective cognitive complaints relate to white matter hyperintensities and future cognitive decline in patients with cardiovascular disease. Am J Geriatr Psychiatry. 2009;17:976-985.

2. Xiong Y-Y, Wong A, Mok VC, Tang W-K, Lam WW, Kwok TC, Chu WC, et al. Frequency and predictors of proxy-confirmed post-stroke cognitive complaints in lacunar stroke patients without major depression. Int J Geriatr Psychiatry. 2011;26:1144-1151.

3. Lamb F, Anderson J, Saling M, Dewey H. Predictors of subjective cognitive complaint in post-acute older adult stroke patients. Arch Phys Med Rehabil. 2013;94:1747-1752.

4. Mascherek A, Zimprich D, Rupprecht R, Lang FR. What do cognitive complaints in a sample of memory clinic outpatients reflect? GeroPsych. 2011;24:187-195.

5. Duits A, Munnecom T, van Heugten C, van Oostenbrugge RJ. Cognitive complaints in the early phase after stroke are not indicative of cognitive impairment. J Neurol Neurosurg Psychiatry. 2008;79:143-146.

6. Jorm AF, Butterworth P, Anstey KJ, Christensen H, Easteal S, Maller J, Mather KA, et al. Memory complaints in a community sample aged 60-64 years: associations with cognitive functioning, psychiatric symptoms, medical conditions, APOE genotype, hippocampus and amygdala volumes, and white-matter hyperintensities. Psychol Med. 2004;34:1495-1506.

7. Miranda B, Madureira S, Verdelho A, Ferro J, Pantoni L, Salvadori E, Chabriat H, et al. Self-perceived memory impairment and cognitive performance in an elderly independent population with age-related white matter changes. J Neurol Neurosurg Psychiatry. 2008;79:869-873.

8. Lam LC, Lui VW, Tam CW, Chiu HF. Subjective memory complaints in Chinese subjects with mild cognitive impairment and early Alzheimer's disease. Int J Geriatr Psychiatry. 2005;20:876-882.

9. Harrington F, Saxby BK, McKeith IG, Wesnes K, Ford GA. Cognitive performance in hypertensive and normotensive older subjects. Hypertension. 2000;36:1079-1082.

10. Waldstein SR. The relation of hypertension to cognitive function. Curr Dir Psychol Sci. 2003;12:9-12.

11. Hannesdottir K, Nitkunan A, Charlton RA, Barrick TR, MacGregor GA, Markus HS. Cognitive impairment and white matter damage in hypertension: a pilot study. Acta Neuro/ Scand. 2009;119:261-268.

12. De Leeuw F-E, de Groot JC, Oudkerk M, Witteman JC, Hofman A, van Gijn J, Breteler MM. Hypertension and cerebral white matter lesions in a prospective cohort study. Brain. 2002;125:765-772.

13. Schulz UG, Rothwell PM. Differences in vascular risk factors between etiological subtypes of ischemic stroke: Importance of population-based studies. Stroke. 2003;34:2050-2059.

14. WardlawJM, Smith EE, Biessels GJ, Cordonnier C, Fazekas F, Frayne R, Lindley RI, et al. Neuroimaging standards for research into small vessel disease and its contribution to ageing and neurodegeneration. Lancet Neurol. 2013;12:822-838.

15. Stewart R, Dufouil C, Godin O, Ritchie K, Maillard P, Delcroix N, Crivello F, et al. Neuroimaging correlates of subjective memory deficits in a community population. Neurology. 2008;70:1601-1607.

16. De Groot JC, de Leeuw F-E, Oudkerk M, Hofman A, Jolles J, Breteler MM. Cerebral white matter lesions and subjective cognitive dysfunction: the Rotterdam Scan Study. Neurology. 2001;56:1539-1545. 
17. Henskens LH, van Oostenbrugge RJ, Kroon AA, de Leeuw PW, Lodder J. Brain microbleeds are associated with ambulatory blood pressure levels in a hypertensive population. Hypertension. 2008;51:62-68.

18. Broadbent DE, Cooper PF, FitzGerald P, Parkes KR. The cognitive failures questionnaire (CFQ) and its correlates. BrJ Clin Psychol. 1982;21:1-16.

19. Brand N, Jolles J. Learning and retrieval rate of words presented auditorily and visually. J Gen Psychol. 1985;112:201-210.

20. Wechsler D. WAIS III, nederlandstalige bewerking: technische handleiding. Lisse: Swets Test Publishers; 2001.

21. Golden CJ. Stroop Colour and Word Test. Chicago (IL): Stoelting; 1978.

22. Reitan R. Trail Making Test: Manual for administration, scoring and interpretation. Bloomington: Indiana University; 1956.

23. Luteyn F. Een nieuwe verkorte GIT. Dutch J Psychol. 1966;2:675-682.

24. Lezak MD, Howieson DB, Loring DW. Neuropsychological assessment. New York: Oxford University Press; 2004.

25. De Koning I, van Kooten F, Koudstaal PJ, Dippel DW. Diagnostic value of the Rotterdam-CAMCOG in post-stroke dementia.J Neurol Neurosurg Psychiatry. 2005;76:263-265.

26. Spinhoven PH, Ormel J, Sloekers PP, Kempen GlJ, Speckens AE, van Hemert AM. A validation study of the Hospital Anxiety and Depression Scale (HADS) in different groups of Dutch subjects. Psychol Med. 1997;27:363-370.

27. Klarenbeek P, van Oostenbrugge RJ, Rouhl RP, Knottnerus IL, Staals J. Ambulatory blood pressure in patients with lacunar stroke: Association with total MRI burden of cerebral small vessel disease. Stroke. 2013;44:29952999.

28. Henskens LH, Kroon AA, van Oostenbrugge RJ, Gronenschild EH, Hofman PA, Lodder J, de Leeuw PW. Associations of ambulatory blood pressure levels with white matter hyperintensity volumes in hypertensive patients. J Hypertens. 2009;27:1446-1452.

29. Narasimhalu K, Wiryasaputra L, Sitoh Y-Y, Kandiah N. Post-stroke subjective cognitive impairment is associated with acute lacunar infarcts in the basal ganglia. Eur J Neurol. 2012;20:547-551.

30. Minett TS, Da Silva RV, Ortiz KZ, Bertolucci PH. Subjective memory complaints in an elderly sample: a cross-sectional study. Int J Geriatr Psychiatry. 2008;23:49-54.

31. Van Norden AG, van Uden IW, de Laat KF, Gons RA, Kessels RP, van Dijk EJ, de Leeuw F-E. Cerebral microbleeds are related to subjective cognitive failures: the RUN DMC study. Neurobiol Aging. 2013;34:2225-2230.

32. Van Norden AG, Fick WF, de Laat KF, van Uden IW, van Oudheusden LJ, Tendolkar I, Zwiers MP, et al. Subjective cognitive failures and hippocampal volume in elderly with white matter lesions. Neurology. 2008;71:1152-1159.

33. Reisberg B, Shulman MB, Torossian C, Leng L, Zhu W. Outcome over seven years of healthy adults with and without subjective cognitive impairment. Alzheimers Dement. 2010;6:11-24.

34. Schmand B, Jonker C, Hooijer C, Lindeboom J. Subjective memory complaints may announce dementia. Neurology. 1996;46:121-125.

35. Dufouil C, Fuhrer R, Alpérovitch A. Subjective cognitive complaints and cognitive decline: consequence or predictor? The epidemiology of vascular aging study. J Am Geriatr Soc. 2005;53:616-621.

36. Watfa G, Rossignol P, Kearney-Schwartz A, Fay R, Bracard S, Felblinger J, Boivin JM, et al. Use of calcium channel blockers is associated with better cognitive performance in older hypertensive patients with subjective memory complaints. J Hypertens. 2010;28:2485-2493. 




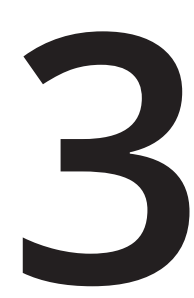

\section{Endothelial activation is associated with cognitive performance in patients with hypertension}

Uiterwijk R, Huijts M, Staals J, Rouhl RPW, de Leeuw PW, Kroon AA, van Oostenbrugge RJ Published in American Journal of Hypertension, 2016; 29: 464-469 


\begin{abstract}
Objectives Hypertension is associated with the occurrence of cognitive deficits and dementia, probably because hypertension is a major risk factor for the occurrence of brain damage as a result of cerebral Small Vessel Disease (cSVD). Endothelial activation and inflammation have been suggested to play an important role in the pathogenesis of CSVD. We investigated if compound scores of endothelial activation or inflammation, based on several blood markers, are associated with cognitive performance three years later in patients with essential hypertension.
\end{abstract}

Methods At baseline, levels of blood markers of endothelial activation (sVCAM-1, sICAM-1, SP-selectin, and sE-selectin) and markers of inflammation (neopterin, C-reactive protein, and sICAM-1) were measured and transformed into compound scores using z-scores. In addition, a brain MRI was performed to determine the presence of CSVD-related MRI markers. Three years later, patients underwent a neuropsychological assessment to determine cognitive performance.

Results 101 patients with hypertension were included in the present study. In multiple linear regression analyses with correction for demographics and MRI markers, the compound score of endothelial activation ( $B=-0.19,95 \% \mathrm{Cl}=-0.34$ to $-0.04, p=0.014)$, but not of inflammation $(B=-0.09,95 \% C l=-0.22$ to $0.05, p=0.198)$, was associated with worse cognitive performance.

Conclusion Our results show that an overall measure of endothelial activation is associated with cognitive performance in patients with essential hypertension. This indicates that a process involving endothelial activation might play a role in the pathogenesis of cognitive problems in patients with hypertension. 


\section{INTRODUCTION}

Hypertension is associated with the occurrence of cognitive deficits and dementia, ${ }^{1}$ probably because hypertension is a major risk factor for the occurrence of brain damage as a result of cerebral Small Vessel Disease (CSVD). ${ }^{2}$ Endothelial activation and inflammation have been suggested to play an important role in the pathogenesis of CSVD. ${ }^{3}$ Studies have shown higher levels of markers of endothelial activation and inflammation in patients with CSVD 4,5 or hypertension ${ }^{6}$ compared with healthy controls. In addition, in Alzheimer's dementia, in which hypertension is also an important vascular risk factor, endothelial activation and inflammation have been suggested to be involved in the pathogenesis. ${ }^{7,8}$

Several studies have investigated associations between cognition and markers of endothelial activation or inflammation and have shown inconsistent results. ${ }^{9-15}$ These studies focused on one or more individual markers, attempting to find biomarkers for predicting cognitive functioning. However, conclusions about the pathogenetic role of endothelial activation or inflammation in general are hard to draw, based on these studies testing single blood markers. By considering multiple blood markers of endothelial activation or inflammation and combining them into one compound score, 16,17 it is possible to draw more general conclusions about the underlying pathogenesis leading to cognitive problems in patients with hypertension.

The aim of the present study is to test the hypothesis that endothelial activation and inflammation, expressed as a compound score of several markers, are associated with cognitive performance three years later in patients with essential hypertension. In addition, we investigated if these associations are independent of possible confounders, in particular, the presence of MRI markers of CSVD.

\section{METHODS}

\section{Study population}

From the hypertension outpatient clinic of the Department of Internal Medicine of Maastricht University Medical Centre, the Netherlands, 218 patients were recruited, for a study on brain damage in patients with essential hypertension (HYBRiD). ${ }^{18}$ Hypertension was defined as an off-medication, clinically measured conventional blood pressure $\geq 140 \mathrm{~mm}$ $\mathrm{Hg}$ systolic or $\geq 90 \mathrm{~mm} \mathrm{Hg}$ diastolic, or both. Details about the HYBRiD study have been described before. ${ }^{18}$ Exclusion criteria were a history of symptomatic cardio- or cerebrovascular disease or contraindications for MRI. Two years after inclusion in the HYBRiD study, patients were asked to participate in the present study. Blood samples were taken, to 
determine levels of markers of endothelial activation and inflammation, and a brain MRI was performed. In addition, three (on-medication) office blood pressure measurements were taken and the mean arterial pressure (MAP) and the pulse pressure (PP) from the second and third measurement were averaged. At that same time point, information about vascular risk factors (body mass index (BMI), smoking, and the presence of diabetes mellitus or hypercholesterolemia) was obtained. Three years after blood sampling and MRI, patients were asked for a follow-up measurement, during which a neuropsychological assessment was performed. This study was approved by the Medical Ethics Committee of the Maastricht University Medical Centre and all participants gave written informed consent.

\section{Markers of endothelial activation and inflammation}

Blood was sampled in the morning after the blood pressure measurement and after an overnight fast. Medication was taken as usual. Blood sampling was performed without a tourniquet, from an antecubital vein, into $5 \mathrm{ml}$ serum and $4 \mathrm{ml}$ (EDTA) plasma tubes (BD Biosciences, Breda, the Netherlands). ${ }^{19}$ Markers of endothelial activation included soluble vascular cellular adhesion molecule-1 (sVCAM-1), soluble intercellular adhesion molecule 1 (sICAM-1), soluble (S)P-selectin and (s)E-selectin. They were all measured using commercially available enzyme-linked immunosorbent assay (ELISA) kits (BioSource Europe, Nivelles, Belgium) according to manufacturer's instructions. For sVCAM-1, sICAM-1, sP-selectin, and sE-selectin, intra-assay variability was 3.1\%, 4.1\%, 2.4\%, and 5.4\%, respectively, whereas inter-assay variability was 5.2\%, 7.7\%,5.2\%, and 6.0\%, respectively. Markers of inflammation included sICAM-1, neopterin and high sensitivity C-reactive protein (hSCRP). sICAM-1 was included in both compound scores, since it is expressed by both monocytes and endothelial cells. ${ }^{20}$ Neopterin was measured using commercially available ELISA kits (IBL Hamburg, Germany), following manufacturer's instructions. Intra- and inter-assay variability were $3.6 \%$ and $7.2 \%$, respectively. hsCRP was determined with nephelometry, using the BN ProSpec (Siemens, Germany); Intra- and inter-assay variability were 1.4\% and $0.9 \%$, respectively.

Levels of individual markers were transformed into standardized values (z-scores), by dividing the difference between the individual score and the sample mean by the sample standard deviation. For each patient, z-scores of sP-selectin, sE-selectin, sICAM-1, and sVCAM-1 were then averaged to an endothelial activation compound score and z-scores of hsCRP, neopterin, and SICAM-1 were averaged to an inflammation compound score. HsCRP levels were missing in 15 patients; inflammation scores of these patients were based on levels of neopterin and sICAM-1 only. 


\section{Neuropsychological assessment}

Cognitive performance was measured with an extensive neuropsychological assessment; as has been described before. ${ }^{21}$ Memory domain was measured with the Rey Auditory Verbal Learning Test22 (immediate recall, delayed recall and delayed recognition) and the Digit Span Forward (subtest of Wechsler Adult Intelligence Scale (WAIS)-III23). Executive function domain was measured with the Stroop Colour Word Test ${ }^{24}$ (SCWT) interference score (time of part 3 minus mean time of parts 1 and 2), Trail Making Test ${ }^{25}$ (TMT) interference score (time of part 2 minus time of part 1), Category (animals and professions) ${ }^{26}$ and Letter Fluency 27, Letter-Number Sequencing (subtest of WAIS-III), and Digit Span Backward (subtest of WAIS-III). Information processing speed domain was measured with the Symbol Substitution - Coding (subtest of WAIS-III), TMT part A, and SCWT parts 1 and 2. Test scores were transformed into standardized values (z-scores), by dividing the difference between the individual raw score and the sample mean by the sample standard deviation. For each patient, domain scores were calculated by averaging these z-scores of the tests within that domain. Finally, the overall cognition score was calculated by averaging the domain scores of memory, executive function and information processing speed.

The Dutch Adult Reading Test (DART) was used as a measure of prior cognitive ability at young age. ${ }^{28}$ The Rotterdam-Cambridge Cognitive Examination (R-CAMCOG) was used to determine the presence of possible dementia, defined as a score $<34 .{ }^{29}$

\section{MRI data}

Four recognized MRI features of CSVD (lacunes, cerebral microbleeds, white matter hyperintensities and perivascular spaces) were measured on baseline brain MRI (standard axial T2-weighted, FLAIR and T2* gradient echo sequences, Intera 1.5-T, Philips Medical Systems, Best, The Netherlands). Two experienced vascular neurologists individually rated the presence of lacunes, cerebral microbleeds, and perivascular spaces in basal ganglia. Definitions and rating method of these lesions have been described before. ${ }^{21}$ White matter hyperintensities volume was semi-automatically assessed using the image-processing software package GIANT, version 2V1.26 (General Imaging and Analysis Tools; Department of Psychiatry and Neuropsychology, Maastricht University, Maastricht, The Netherlands). ${ }^{30}$ A trained observer performed the quantitative assessments after reaching excellent interobserver agreement with an experienced neuroradiologist.

\section{Statistical analysis}

IBM SPSS Statistics 20 software was used for all analyses. The associations between endothelial activation or inflammation compound scores (predictor) and overall cognition (dependent variable) were assessed with simple linear regression analyses. Additionally, we corrected these associations for possible confounders. In model 1, the associations 
were adjusted for demographics (sex, age and DART score). In model 2, the associations were adjusted for demographics and vascular risk factors (diabetes mellitus, hypercholesterolemia, smoking, BMI, PP and MAP). In model 3, the associations were adjusted for demographics and all of the MRI markers of CSVD (white matter hyperintensities volume and the presence of lacunes, cerebral microbleeds and perivascular spaces). Our sample size precluded a model with all predictors (demographics, vascular risk factors and MRI markers of (SVD), as this would make the number of predictors in the model too high. Stepwise analyses of the multiple regression analyses models 1, 2 and 3 were performed to investigate how much of the variance in cognitive function was explained by endothelial activation score or inflammation score additional to the covariates. We also tested for interaction effects between MRI markers of CSVD and endothelial activation or inflammation compound scores on overall cognition. Simple and multiple regression analyses were repeated with domain scores of memory, executive function or information processing speed as dependent variable. Results were considered significant at $p<0.05$.

Figure 1. Flow of participants

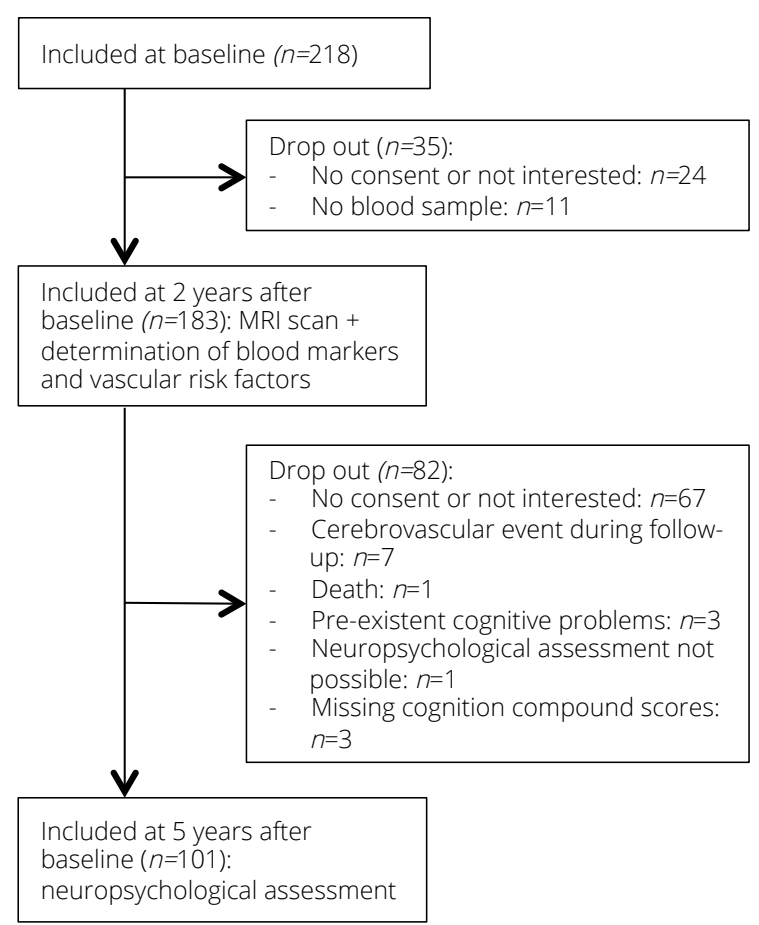




\section{RESULTS}

Of the original 218 patients in the HYBRiD study, 183 patients were included in the present study that started 2 years later and 104 patients completed the follow-up 3 years later. Three patients were excluded from analyses because cognition compound scores were missing. Of one patient the DART score was missing. Imputation of the mean was used for this missing value to prevent exclusion of this patient. Flow of participants is shown in figure 1. The included patients $(n=101)$ did not differ from the excluded patients $(n=117)$ in age $(54.7 \pm 11.5$ versus $54.3 \pm 13.6, p=0.819)$, but included patients were more often male (60.4\% versus $42.5 \%, p=0.008)$. Patients' characteristics are shown in table 1 . One patient had an R-CAMCOG score <34, which indicates possible dementia.

Table 1. Patients' characteristics

\begin{tabular}{|c|c|}
\hline Characteristic & All patients $(n=101)$ \\
\hline Age (years, SD) & $54.7(11.5)$ \\
\hline Male sex (\%) & $61(60.4)$ \\
\hline DART score (SD) & $82.6(1.4)$ \\
\hline Median R-CAMCOG (IQR) & $43(39-47)$ \\
\hline Diabetes Mellitus (\%) & $4(4.0)$ \\
\hline Hypercholesterolemia (\%) & $40(39.6)$ \\
\hline Current smoking (\%) & $18(17.8)$ \\
\hline Body Mass Index (kg/m², SD) & $28.0(4.3)$ \\
\hline MAP (mmHG, SD) & $108.3(11.9)$ \\
\hline Pulse Pressure (mmHG, SD) & $54.5(14.7)$ \\
\hline Antihypertensive medication use (\%) & $90(89.1)$ \\
\hline \multicolumn{2}{|l|}{ Blood markers } \\
\hline Median of hsCRP $(\mathrm{mg} / \mathrm{L}, I Q R)^{\star}$ & $1.8(0.84-3.8)$ \\
\hline Median of Neopterin $(\mathrm{ng} / \mathrm{mL}, I Q R)$ & $1.5(1.3-1.8)$ \\
\hline Median of sP-selectin (ng/mL, IQR) & 35.7 (22.0-61.5) \\
\hline Median of sE-selectin (ng/mL, IQR) & $22.1(17.2-34.0)$ \\
\hline Median of sICAM-1 (ng/mL, IQR) & $428.4(378.1-537.2)$ \\
\hline Median of sVCAM-1 (ng/mL, IQR) & $677.2(567.0-760.9)$ \\
\hline \multicolumn{2}{|l|}{ MRI markers } \\
\hline Median of WMH volume $\left(\mathrm{mm}^{3}, I Q R\right)$ & $666.9(369.3-1,553.8)$ \\
\hline PVS basal ganglia (\%) & $33(32.7 \%)$ \\
\hline Cerebral microbleeds (\%) & $12(11.9 \%)$ \\
\hline Lacunes (\%) & $6(6.0 \%)$ \\
\hline
\end{tabular}

$S D=$ standard deviation; $I Q R=$ interquartile range; $M A P=$ mean arterial pressure; $W M H=$ white matter hyperintensities; PVS=perivascular spaces

* $n=86$ 
In unadjusted regression analyses, overall cognition was significantly associated with compound scores of endothelial activation and inflammation (table 2). The association between endothelial activation score and overall cognition remained significant after correction for age, sex, and DART score (model 1), as well as after additional correction for vascular risk factors (model 2) or MRI markers (model 3). In model 1, age, sex and DART score explained $61 \%$ of the variance in cognitive performance. In model 1, 2 and 3, respectively $2.7 \%, 2.7 \%$ and $2.4 \%$ of the variance in cognitive performance was explained by endothelial activation additional to the covariates in these models. The association between inflammation score and overall cognition was no longer significant after adjustment for age, sex, and DART score (model 1). In model 1, 2 and 3 of the multiple regression analyses, respectively $0.8 \%$, $1.0 \%$ and $0.7 \%$ of the variance in cognitive performance was explained by inflammation. No interaction effects were found between MRI markers of CSVD and endothelial activation or inflammation score on overall cognition (results not shown).

Table 2. Association between endothelial activation and inflammation scores and overall cognition score

\begin{tabular}{llllll}
\hline & $\begin{array}{l}\text { Endothelial Activation } \\
\boldsymbol{B}(95 \% \mathrm{Cl})\end{array}$ & $\boldsymbol{p}$ & $\begin{array}{l}\text { Inflammation } \\
\boldsymbol{B}(95 \% \mathrm{Cl})\end{array}$ & $\boldsymbol{p}$ \\
\hline Simple linear regression & $-0.39(-0.60$ to -0.19$)$ & $p<0.001$ & $-0.22(-0.42$ to -0.01$)$ & $p=0.038$ \\
Multiple linear regression & Model 1 & $-0.19(-0.34$ to -0.05$)$ & $p=0.008$ & $-0.10(-0.23$ to 0.04$)$ & $p=0.155$ \\
& Model 2 & $-0.20(-0.35$ to -0.05$)$ & $p=0.008$ & $-0.11(-0.25$ to 0.03$)$ & $p=0.116$ \\
& Model 3 & $-0.19(-0.34$ to -0.04$)$ & $p=0.014$ & $-0.09(-0.22$ to 0.05$)$ & $p=0.198$ \\
\hline
\end{tabular}

$B=$ unstandardized regression coefficient; $\mathrm{Cl}=$ confidence interval Model 1: adjusted for age, sex and DART score

Model 2: adjusted for age, sex, DART score and vascular risk factors

Model 3: adjusted for age, sex, DART score and MRI markers

Associations between endothelial activation score and the domain scores of executive function and information processing speed were significant in the simple regression analyses as well as in the adjusted regression analyses (table 3), except for the association between endothelial activation and information processing speed additionally corrected for MRI markers. Memory was not associated with endothelial activation in the adjusted regression analyses. Associations between inflammation score and memory, executive function and information processing speed were not significant in the adjusted regression analyses (table 3). Associations between overall cognition and all individual blood markers can be found in the supplementary material (table S1). 
Table 3. Association between endothelial activation and inflammation scores and cognitive domains

\begin{tabular}{|c|c|c|c|c|c|}
\hline & & $\begin{array}{l}\text { Endothelial Activation } \\
B(95 \% \mathrm{Cl})\end{array}$ & $p$ & $\begin{array}{l}\text { Inflammation } \\
B(95 \% \mathrm{Cl})\end{array}$ & $p$ \\
\hline \multicolumn{6}{|l|}{ Memory } \\
\hline Simple linear regression & & $-0.33(-0.56$ to -0.11$)$ & $p=0.004$ & $-0.17(-0.39$ to 0.05$)$ & $p=0.124$ \\
\hline \multirow[t]{3}{*}{ Multiple linear regression } & Model 1 & $-0.15(-0.34$ to 0.05$)$ & $p=0.145$ & $-0.08(-0.27$ to 0.10$)$ & $p=0.369$ \\
\hline & Model 2 & $-0.16(-0.36$ to 0.04$)$ & $p=0.124$ & $-0.09(-0.28$ to 0.10$)$ & $p=0.339$ \\
\hline & Model 3 & $-0.15(-0.35$ to 0.06$)$ & $p=0.159$ & $-0.08(-0.26$ to 0.11$)$ & $p=0.395$ \\
\hline \multicolumn{6}{|l|}{ Executive function } \\
\hline Simple linear regression & & $-0.41(-0.63$ to -0.19$)$ & $p<0.001$ & $-0.26(-0.48$ to -0.04$)$ & $p=0.020$ \\
\hline \multirow[t]{3}{*}{ Multiple linear regression } & Model 1 & $-0.23(-0.40$ to -0.07$)$ & $p=0.008$ & $-0.13(-0.29$ to 0.03$)$ & $p=0.104$ \\
\hline & Model 2 & $-0.24(-0.42$ to -0.07$)$ & $p=0.008$ & $-0.16(-0.32$ to 0.01$)$ & $p=0.071$ \\
\hline & Model 3 & $-0.22(-0.40$ to -0.04$)$ & $p=0.015$ & $-0.12(-0.28$ to 0.04$)$ & $p=0.137$ \\
\hline \multicolumn{6}{|c|}{ Information processing speed } \\
\hline Simple linear regression & & $-0.43(-0.69$ to -0.16$)$ & $p=0.002$ & $-0.22(-0.48$ to 0.04$)$ & $p=0.101$ \\
\hline \multirow[t]{3}{*}{ Multiple linear regression } & Model 1 & $-0.21(-0.40$ to -0.02$)$ & $p=0.035$ & $-0.08(-0.25$ to 0.10$)$ & $p=0.390$ \\
\hline & Model 2 & $-0.20(-0.39$ to -0.01$)$ & $p=0.042$ & $-0.09(-0.27$ to 0.09$)$ & $p=0.324$ \\
\hline & Model 3 & $-0.19(-0.39$ to 0.01$)$ & $p=0.061$ & $-0.06(-0.24$ to 0.12$)$ & $p=0.480$ \\
\hline
\end{tabular}

$B=$ unstandardized regression coefficient; $\mathrm{Cl}=$ confidence interval Model 1: adjusted for age, sex and DART score

Model 2: adjusted for age, sex, DART score and vascular risk factors

Model 3: adjusted for age, sex, DART score and MRI markers

\section{DISCUSSION}

We showed that endothelial activation, represented by a compound score of several blood markers for endothelial function, is associated with cognitive performance three years later in patients with hypertension, also after controlling for MRI markers of CSVD or vascular risk factors. In addition, results showed that $2.7 \%$ of the variance in cognitive performance was explained by endothelial activation after controlling for demographics and vascular risk factors. In contrast, we could not find an independent association between inflammation score and cognitive performance.

Previous studies have reported associations between cognitive performance and levels of several individual markers, such as CRP, haptoglobin, plasma fibrinogen, interleukin-6, and sICAM-1, 10-12 whereas other studies failed to show associations with these and other markers. ${ }^{12,13}$ We examined the association with a compound score of markers, which enables us to draw conclusions about the overall effect of endothelial activation and inflammation 
instead of a diversity of conclusions based on individual markers. Furthermore, the use of compound scores reduces the effect of biological variability of each blood marker and reduces the number of investigated associations and thereby minimizes problems of multiple testing.

We could find only one other recent study ${ }^{16}$ that investigated this association between cognitive performance and inflammation or endothelial activation, in a sample of elderly people using compound scores of blood markers. In this study, an association of both inflammation and endothelial dysfunction with worse cognitive performance on the information processing speed domain and the attention and executive function domain was shown. ${ }^{16}$ We only showed an association between cognition and endothelial activation, which suggests that endothelial activation is primary involved in the pathogenesis leading to cognitive problems in patients with hypertension. Disruption of the blood-brain barrier might be such an endothelium mediated process. ${ }^{31}$ The results of our study also support the vascular hypothesis of cognitive decline and are in agreement with other studies showing elevated levels of markers of endothelial activation in patients with Alzheimer's disease. ${ }^{32,33}$ The partly contradicting results of our study and the previous one ${ }^{16}$ involving inflammation could possibly be explained by the different populations that were investigated or by the small sample size of our study (101 patients) in contrast to the 363 patients included in the previous mentioned study. The post-hoc calculated power of the observed small effect size for the association between inflammation and cognitive performance (effect size $f^{2}=0.021$, in multiple regression with correction for age, sex and DART score) was only 30\%. In contrast, our multiple regression analysis investigating the association between endothelial activation and cognitive performance showed an effect size $\left(f^{2}\right)$ of 0.074 , which established a power of $77.4 \%$

Strengths of our study include the extensive neuropsychological assessment and accounting for the presence of MRI markers of CSVD. These MRI markers might be causal intermediates in the association between endothelial activation or inflammation and cognitive performance. However, we have shown that the association between endothelial activation and cognition still exists after correction for MRI markers, and the regression coefficient of endothelial activation or inflammation even hardly changed in the model including the MRI markers. Therefore, we can conclude that this association is not dependent on the presence of macrostructural brain damage. There must be other pathways present between endothelial activation and cognitive performance. For example, microstructural damage, not visible on the used MRI sequences, or other processes than CSVD might have considerable effect on cognitive function. Similarly, endothelial activation was associated with cognitive performance, independently of vascular risk factors. Although the influence of vascular risk factors on endothelial activation is considered important, our 
results implicate that others factors, besides vascular risk factors, influence endothelial activation.

A limitation of the study is the lack of neuropsychological assessment at baseline, which withheld us from determining cognitive decline. However, since we used the DART to control for prior cognitive performance, results actually reflect associations with a lifetime change in cognitive performance. Secondly, information about the hypertension status or treatment at the time of the neuropsychological assessment was not available. Thirdly, since we included only 101 patients of the 218 patients of the previous study, this might have caused a certain selection bias. Last, all markers were equally weighted in the compound scores of endothelial activation and inflammation, but it is unsure whether they really all are equally important in the process of endothelial activation or inflammation. Furthermore, there might be other blood markers of interest that we did not measure. However, the circulating adhesion molecules that we used are believed to serve as markers for the detection of general endothelial activation ${ }^{34}$ and therefore give an overall insight into endothelial activation. We used hSCRP, neopterin and SICAM-1 to assess inflammation; these markers globally encompass the process of inflammation (leukocyte adhesion and activation). In addition, inflammation and endothelial activation markers were measured only once and intra-individual fluctuations cannot be excluded. However, markers were measured in a non-active phase of a chronic condition, so levels are expected to remain relatively stable.

In conclusion, our study shows that a compound score of endothelial activation is associated with cognitive performance in patients with hypertension. These results suggest that a process of endothelial activation might be involved in the pathogenesis of cognitive problems in patients with hypertension. Future research is necessary to further investigate the role of endothelial activation in processes causing cognitive problems in these patients. 


\section{REFERENCES}

1. Iadecola C. Hypertension and dementia. Hypertension. 2014;64:3-5.

2. Veglio F, Paglieri C, Rabbia F, Bisbocci D, Bergui M, Cerrato P. Hypertension and cerebrovascular damage. Atherosclerosis. 2009;205:331-341.

3. Wardlaw JM, Smith C, Dichgans M. Mechanisms of sporadic cerebral small vessel disease: insights from neuroimaging. Lancet Neurol. 2013;12:483-497.

4. Van Dijk EJ, Prins ND, Vermeer SE, Vrooman HA, Hofman A, Koudstaal PJ, Breteler MMB. C-reactive protein and cerebral small-vessel disease: the Rotterdam Scan Study. Circulation. 2005;112:900-905.

5. Hassan A, Hunt BJ, O'Sullivan M, Parmar K, Bamford JM, Briley D, Brown MM, et al. Markers of endothelial dysfunction in lacunar infarction and ischaemic leukoaraiosis. Brain. 2003;126:424-432.

6. Boulbou MS, Koukoulis GN, Makri ED, Petinaki EA, Gourgoulianis KI, Germenis AE. Circulating adhesion molecules levels in type 2 diabetes mellitus and hypertension. Int J Cardiol. 2005;98:39-44.

7. Kelleher RJ, Soiza RL. Evidence of endothelial dysfunction in the development of Alzheimer's disease: Is Alzheimer's a vascular disorder? Am J Cardiovasc Dis. 2013;3:197-226.

8. Takeda S, Sato N, Morishita R. Systemic inflammation, blood-brain barrier vulnerability and cognitive/ non-cognitive symptoms in Alzheimer disease: relevance to pathogenesis and therapy. Front Aging Neurosci. 2014;6:171

9. Umemura T, Kawamura T, Umegaki H, Mashita S, Kanai A, Sakakibara T, Hotta N, et al. Endothelial and inflammatory markers in relation to progression of ischaemic cerebral small-vessel disease and cognitive impairment: a 6-year longitudinal study in patients with type 2 diabetes mellitus. J Neurol Neurosurg Psychiatry. 2011;82:1186-1194

10. Teunissen C, Van Boxtel M, Bosma H, Bosmans E, Delanghe J, De Bruijn C, Wauters A, et al. Inflammation markers in relation to cognition in a healthy aging population. J Neuroimmunol. 2003;134:142-150.

11. Gunstad J, Bausserman L, Paul RH, Tate DF, Hoth K, Poppas A, Jefferson AL, et al. C-reactive protein, but not homocysteine, is related to cognitive dysfunction in older adults with cardiovascular disease. J Clin Neurosci. 2006;13:540-546

12. Rafnsson SB, Deary IJ, Smith FB, Whiteman MC, Rumley A, Lowe G, Fowkes FGR. Cognitive decline and markers of inflammation and hemostasis: the Edinburgh Artery Study. J Am Geriatr Soc. 2007;55:700-707.

13. Miralbell J, Soriano JJ, Spulber G, López-Cancio E, Arenillas JF, Bargalló N, Galán A, et al. Structural brain changes and cognition in relation to markers of vascular dysfunction. Neurobiol Aging. 2012;33:1003.e9-1003.e17.

14. Quinn T, Gallacher J, Deary I, Lowe G, Fenton C, Stott D. Association between circulating hemostatic measures and dementia or cognitive impairment: systematic review and meta-analyzes. J Thromb Haemost. 2011;9:14751482.

15. Obasi CN, Cruickshanks KJ, Nondahl DM, Klein BE, Klein R, Nieto FJ, Shankar A, et al. Association of biomarkers for inflammation, endothelial dysfunction and oxidative stress with cognitive impairment. The Epidemiology of Hearing Loss Study (EHLS). Oxid Antioxid Med Sci. 2012;1:169-173.

16. Heringa S, van den Berg E, Reijmer Y, Nijpels G, Stehouwer C, Schalkwijk C, Teerlink T, et al. Markers of lowgrade inflammation and endothelial dysfunction are related to reduced information processing speed and 
executive functioning in an older population: The Hoorn Study. Psychoneuroendocrinology. 2014;40:108-118.

17. Aribisala BS, Wiseman S, Morris Z, Valdés-Hernández MC, Royle NA, Maniega SM, Gow AJ, et al. Circulating inflammatory markers are associated with magnetic resonance imaging-visible perivascular spaces but not directly with white matter hyperintensities. Stroke. 2014;45:605-607.

18. Henskens LH, van Oostenbrugge RJ, Kroon AA, de Leeuw PW, Lodder J. Brain microbleeds are associated with ambulatory blood pressure levels in a hypertensive population. Hypertension. 2008;51:62-68.

19. Rouhl RP, Damoiseaux JG, Lodder J, Theunissen RO, Knottnerus IL, Staals J, Henskens LH, et al. Vascular inflammation in cerebral small vessel disease. Neurobiol Aging. 2012;33:1800-1806.

20. Schram M, Stehouwer C. Endothelial dysfunction, cellular adhesion molecules and the metabolic syndrome. Horm Metab Res. 2005;37:49-55.

21. Uiterwijk R, Huijts M, Staals J, Duits A, Gronenschild E, Kroon AA, de Leeuw PW, et al. Subjective cognitive failures in patients with hypertension are related to cognitive performance and cerebral microbleeds. Hypertension. 2014;64:653-657.

22. Brand N, Jolles J. Learning and retrieval rate of words presented auditorily and visually. J Gen Psychol. 1985;112:201-210.

23. Wechsler D. WAIS III, nederlandstalige bewerking: technische handleiding. Lisse: Swets Test Publishers; 2001.

24. Golden CJ. Stroop Colour and Word Test. Chicago (IL): Stoelting; 1978.

25. Reitan R. Trail Making Test: Manual for administration, scoring and interpretation. Bloomington: Indiana University; 1956.

26. Luteyn F. Een nieuwe verkorte GIT. Dutch J Psychol. 1966;2:675-682.

27. Lezak MD, Howieson DB, Loring DW. Neuropsychological assessment. New York: Oxford University Press; 2004.

28. Schmand B, Bakker D, Saan R, Louman J. The Dutch Reading Test for Adults: a measure of premorbid intelligence level. Tijdschr Gerontol Geriatr. 1991;22:15-19.

29. De Koning I, van Kooten F, Koudstaal PJ, Dippel DW. Diagnostic value of the Rotterdam-CAMCOG in post-stroke dementia.J Neurol Neurosurg Psychiatry. 2005;76:263-265.

30. Henskens LH, Kroon AA, van Oostenbrugge RJ, Gronenschild EH, Hofman PA, Lodder J, de Leeuw PW. Associations of ambulatory blood pressure levels with white matter hyperintensity volumes in hypertensive patients. J Hypertens. 2009;27:1446-1452.

31. Oakley R, Tharakan B. Vascular hyperpermeability and aging. Aging Dis. 2014;5:114-125.

32. Zuliani G, Cavalieri M, Galvani M, Passaro A, Munari M, Bosi C, Zurlo A, et al. Markers of endothelial dysfunction in older subjects with late onset Alzheimer's disease or vascular dementia. J Neurol Sci. 2008;272:164-170.

33. Borroni B, Volpi R, Martini G, Del Bono R, Archetti S, Colciaghi F, Akkawi NM, et al. Peripheral blood abnormalities in Alzheimer disease: evidence for early endothelial dysfunction. Alzheimer Dis Assoc Disord. 2002;16:150-155.

34. Paulus P, Jennewein C, Zacharowski K. Biomarkers of endothelial dysfunction: can they help us deciphering systemic inflammation and sepsis? Biomarkers. 2011;16:S11-S21. 


\section{SUPPLEMENTARY MATERIAL}

Table S1. Associations between individual marker levels and overall cognition

\begin{tabular}{|c|c|c|c|c|}
\hline & & $\begin{array}{l}\text { CRP } \\
B(95 \% \mathrm{Cl})\end{array}$ & $\begin{array}{l}\text { Neopterin } \\
B(95 \% \mathrm{Cl})\end{array}$ & $\begin{array}{l}\text { P-selectin } \\
B(95 \% \mathrm{Cl})\end{array}$ \\
\hline \multirow{2}{*}{\multicolumn{2}{|c|}{ Simple regression }} & $0.008(-0.02$ to 0.03$)$ & $-0.25(-0.49$ to 0.003$)$ & $-0.002(-0.004$ to 0.001$)$ \\
\hline & & $p=0.53$ & $p=0.052$ & $p=0.28$ \\
\hline \multirow{6}{*}{$\begin{array}{l}\text { Multiple linear } \\
\text { regression }\end{array}$} & \multirow[t]{2}{*}{ Model 1} & $-0.001(-0.02$ to 0.01$)$ & $-0.04(-0.20$ to 0.13$)$ & $-0.002(-0.004$ to 0.000$)$ \\
\hline & & $p=0.85$ & $p=0.68$ & $p=0.017$ \\
\hline & \multirow[t]{2}{*}{ Model 2} & $-0.002(-0.02$ to 0.01$)$ & $-0.04(-0.22$ to 0.13$)$ & $-0.003(-0.005$ to -0.001$)$ \\
\hline & & $p=0.79$ & $p=0.63$ & $p=0.007$ \\
\hline & \multirow[t]{2}{*}{ Model 3} & $-0.003(-0.02$ to 0.01$)$ & $-0.02(-0.15$ to 0.10$)$ & $-0.002(-0.004$ to -0.001$)$ \\
\hline & & $p=0.74$ & $p=0.74$ & $p=0.008$ \\
\hline \multicolumn{5}{|c|}{ Table S1 continued } \\
\hline & & $\begin{array}{l}\text { E-selectin } \\
B(95 \% \mathrm{Cl})\end{array}$ & $\begin{array}{l}\text { ICAM } \\
\text { B }(95 \% \mathrm{Cl})\end{array}$ & $\begin{array}{l}\text { VCAM } \\
\text { B }(95 \% \mathrm{Cl})\end{array}$ \\
\hline \multirow{2}{*}{\multicolumn{2}{|c|}{ Simple regression }} & $0.000(-0.008$ to 0.007$)$ & $-0.002(-0.003$ to -0.001$)$ & $-0.001(-0.002$ to 0.000$)$ \\
\hline & & $p=0.93$ & $P<0.001$ & $p=0.033$ \\
\hline \multirow{6}{*}{$\begin{array}{l}\text { Multiple linear } \\
\text { regression }\end{array}$} & \multirow[t]{2}{*}{ Model 1} & $0.000(-0.005$ to 0.005$)$ & $-0.001(-0.001$ to 0.000$)$ & $0.000(-0.001$ to 0.000$)$ \\
\hline & & $p=0.96$ & $p=0.021$ & $p=0.20$ \\
\hline & \multirow[t]{2}{*}{ Model 2} & $0.001(-0.004$ to 0.006$)$ & $-0.001(-0.001$ to 0.000$)$ & $0.000(-0.001$ to 0.000$)$ \\
\hline & & $p=0.67$ & $p=0.019$ & $p=0.12$ \\
\hline & \multirow[t]{2}{*}{ Model 3} & $0.000(-0.005$ to 0.006$)$ & $-0.001(-0.001$ to 0.000$)$ & $0.000(-0.001$ to 0.000$)$ \\
\hline & & $p=0.88$ & $p=0.040$ & $p=0.28$ \\
\hline
\end{tabular}

$B=$ unstandardized regression coefficient; $\mathrm{Cl}=$ confidence interval Model 1: adjusted for age, sex and DART score Model 2: adjusted for age, sex, DART score and vascular risk factors Model 3: adjusted for age, sex, DART score and MRI markers 




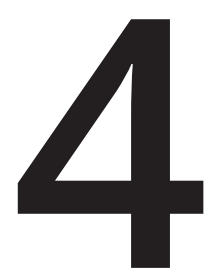

Framingham stroke risk profile is related to cerebral small vessel disease progression and lower cognitive performance in patients with hypertension

Uiterwijk R, Staals J, Huijts M, de Leeuw PW, Kroon AA, van Oostenbrugge RJ 


\section{ABSTRACT}

Objectives The Framingham stroke risk profile (FSRP) was developed to predict clinical stroke. We investigated if FSRP is associated with more 'silent' effects of cerebrovascular disease, namely progression of cerebral Small Vessel Disease (cSVD)-related brain damage and cognitive performance in hypertensive patients.

Methods 90 patients with essential hypertension underwent a brain MRI scan and FSRP assessment at baseline, and a second brain MRI scan and neuropsychological assessment at 9-year follow-up. We visually rated progression of cSVD-related MRI markers.

Results FSRP was associated with progressive periventricular white matter hyperintensities ( $p=0.017$ ) and new microbleeds ( $p=0.031$ ), but not after correction for the FSRP age component. FSRP was associated with lower overall cognitive performance $(p<0.001)$ and this remained significant after correction for the FSRP age component.

Conclusion A vascular risk score might be useful in predicting progression of cSVD-related brain damage or future cognitive performance in hypertensive patients. Age seems to be the most important component in FSRP. 


\section{INTRODUCTION}

Cerebral small vessel disease (CSVD) is a pathologic process involving the small arteries and arterioles in the brain and resulting in MRI abnormalities, such as white matter hyperintensities (WMH), lacunes and microbleeds.' ${ }^{1}$ CSVD is a major cause of cognitive deficits and cognitive decline. ${ }^{2,3}$ Hypertension is probably the most important risk factor for CSVD. ${ }^{4}$ Other cardiovascular risk factors, such as diabetes mellitus and smoking, have also been associated with CSVD ${ }^{5-7}$ and lower cognitive function. ${ }^{8-10}$

The Framingham stroke risk profile (FSRP) is a composite vascular risk score, which was originally developed to predict clinical stroke. Previous studies have investigated whether the FSRP is also associated with more 'silent' cerebrovascular disease, such as MRI markers of CSVD. Several studies have shown that an increased FSRP is associated with the extent of WMH. ${ }^{11,12}$ Other studies showed a higher FSRP in patients with silent cerebral infarcts or microbleeds compared to patients without these lesions..$^{13-15}$ Even though the FSRP is a prediction score, no longitudinal studies examining the association with progression of CSVD or new CSVD lesions could be found.

A number of cross-sectional and longitudinal studies have examined the association between FSRP and cognitive performance or cognitive decline. For example, in the Framingham Offspring Cohort, an inverse association between FSRP and cognitive performance in several domains was shown. ${ }^{16}$ Another study showed the FSRP to be associated with greater global cognitive decline over 10 years. ${ }^{17}$

Studies investigating the associations between FSRP and progressive CSVD and cognitive performance in patients with hypertension are lacking. More insight into the use of this composite vascular risk score in this population at high risk of CSVD and cognitive dysfunction is needed. Therefore, we investigated if the FSRP is associated with progression of MRI markers of CSVD and cognitive performance in patients with essential hypertension, in a longitudinal, 9-year follow-up study.

\section{METHODS}

\section{Study population}

Subjects were essential hypertensive patients who were included in a study on brain damage in patients with essential hypertension (HYBRiD). ${ }^{18}$ Patients were recruited from the hypertension outpatient clinic of the Department of Internal Medicine of Maastricht University Medical Centre, the Netherlands. Hypertension was defined as an off-med- 
ication, clinically measured conventional blood pressure $\geq 140 \mathrm{~mm} \mathrm{Hg}$ systolic or $\geq 90$ $\mathrm{mm} \mathrm{Hg}$ diastolic, or both. Details about the HYBRiD study have been described before. ${ }^{18}$ Exclusion criteria were documented diabetes, ischemic or valvular heart disease, electrocardiographic evidence of atrial fibrillation, history of transient ischemic attacks or stroke and obstructive sleep apnea syndrome. At baseline, all patients received a brain MRI and the FSRP was determined. Nine years later, patients were invited for a second brain MRI and a neuropsychological assessment. The current study is a retrospective, secondary analysis of the HYBRiD study. The Medical Ethics Committee of the Maastricht University Medical Centre approved this study and all participants gave written informed consent.

\section{Framingham Stroke Risk Profile}

The FSRP is developed to predict 10-year probability of stroke and is derived from the Framingham Study. ${ }^{19,20}$ The gender-specific score is composed of age, systolic blood pressure (SBP) with or without antihypertensive medication, diabetes, cigarette smoking status, history of cardiovascular disease, atrial fibrillation and left ventricular hypertrophy. In our study, the FSRP factors were determined at baseline. SBP was determined by off-medication, conventional office blood measurements by sphygmomanometry. After at least five minutes of rest, three consecutive measurements were taken at the non-dominant arm, with the participant seated. The mean of the second and third measurements was calculated. Electrocardiographic left ventricular hypertrophy was defined as a Sokolow-Lyons index $\left(S V_{1}+R V_{5-6}\right)>38 \mathrm{~mm}$. Since diabetes mellitus and atrial fibrillation were exclusion criteria for our study, all patients received 0 points for these factors in the FSRP.

\section{Neuropsychological assessment}

Cognitive performance was measured at 9-year follow-up with an extensive neuropsychological assessment; as has been described before. ${ }^{21}$ Memory domain was measured with the Rey Auditory Verbal Learning Test 22 (immediate recall, delayed recall and delayed recognition) and the Digit Span Forward (subtest of Wechsler Adult Intelligence Scale (WAIS)$\|^{\prime 23}$ ). Executive function domain was measured with the Stroop Colour Word Test $^{24}$ (SCWT) interference score (time of part 3 minus mean time of parts 1 and 2), Trail Making Test ${ }^{25}$ (TMT) interference score (time of part 2 minus time of part 1), Category (animals and professions) ${ }^{26}$ and Letter Fluency, ${ }^{27}$ Letter-Number Sequencing (subtest of WAIS-III), and Digit Span Backward (subtest of WAIS-III). Information processing speed domain was measured with the Symbol Substitution - Coding (subtest of WAIS-III), TMT part A, and SCWT parts 1 and 2. Test scores were transformed into standardized values (z-scores), by dividing the difference between the individual raw score and the sample mean by the sample standard deviation. For each patient, domain scores were calculated by averaging these z-scores of the tests within that domain. Finally, the overall cognition score was calculated by averaging the domain scores of memory, executive function and information processing speed. 
The Dutch Adult Reading Test (DART) was used as a measure of prior cognitive ability. ${ }^{28}$ The Rotterdam-Cambridge Cognitive Examination (R-CAMCOG) was used to determine the presence of possible dementia, defined as a score $<34 .{ }^{29}$ The Hospital Anxiety and Depression Scale (HADS) total score (range 0-42) was used to determine symptoms of depression and anxiety. Since symptoms possibly caused by physical problems (e.g. insomnia or weight loss) are not included in the HADS, the scale is considered to be suitable to use in somatic populations. ${ }^{30}$

\section{MRI data}

On brain MRI scans at baseline and after 9 years (standard axial T2-weighted, FLAIR and T2* gradient echo sequences, Intera 1.5-T, Philips Medical Systems, Best, The Netherlands) two experienced vascular neurologists rated baseline MRI markers, and one rated progression of markers, after reaching satisfactory inter-rater agreements. The interrater agreement statistics have been previously reported. ${ }^{31}$ Baseline WMH were assessed according to the Fazekas scale. ${ }^{32}$ Presence of extensive periventricular $\mathrm{WMH}$ was defined as periventricular WMH Fazekas score 3 (irregular hyperintensities extending into the deep matter) and presence of extensive subcortical/deep WMH was defined as deep WMH Fazekas score 2 or 3 (confluent hyperintensities). Lacunes and cerebral microbleeds were assessed according to international consensus definition. ${ }^{33}$ An overall score for the baseline MRI total burden of cSVD was composed as described earlier. ${ }^{34}$

Progression of periventricular and subcortical WMH were scored according to the WMH change scale as proposed by Prins et al. ${ }^{35}$ Progression of periventricular WMH was scored in three regions (frontal caps, lateral band and occipital caps) and progression of subcortical WMH is scored in four regions (frontal, parietal, temporal and occipital). New lesions or increase of existing lesions was scored in each region as +1 and disappeared lesions or decrease of existing lesions was scored as -1 . This adds up to a progression score ranging from -3 to +3 for periventricular WMH and -4 to +4 for subcortical WMH. We defined progression of periventricular or subcortical $\mathrm{WMH}$ as a score of $\geq 1$. Progression of lacunes or microbleeds was defined as any new lacune or microbleed.

\section{Statistical analysis}

Associations between FSRP and progression of MRI markers of cSVD were analysed with simple logistic regression and thereafter with correction for baseline presence of the studied MRI marker. Associations between FSRP and cognitive performance in overall cognition and three separate cognitive domains (executive function, information processing speed and memory) were first analysed with simple linear regression analyses and second with multivariable linear regression with correction for DART and HADS score. 
Exploratory analyses were performed to examine if associations between FSRP and progression of MRI markers or cognitive performance were explained by just the age or SBP component, as these are known to be important determinants of CSVD and cognition. Therefore, the associations between FSRP and progression of MRI markers (including correction for the baseline MRI marker) that were found to be significant were additionally adjusted for the FSRP age or SBP component (i.e. the points awarded in the FSRP for age or SBP). Similarly, the significant associations between FSRP and cognitive performance, with correction for DART and HADS, were additionally adjusted for the age and SBP FSRP component separately. We also performed exploratory analyses in which we adjusted the associations between FSRP and cognitive performance for baseline total burden of CSVD on MRI.

IBM SPSS Statistics 22 software was used for all analyses. Results were considered significant at $p<0.05$.

\section{RESULTS}

\section{Participants}

Of the original 218 patients in the HYBRiD study, 90 (41.3\%) patients completed the follow-up after 9 years. Reasons for exclusion during follow-up were: no consent to be contacted for follow-up ( $n=20)$, not interested $(n=79)$, cerebrovascular event during follow-up ( $n=7)$, death ( $n=4)$, contra-indications for MRI $(n=5)$, neuropsychological assessment not possible $(n=4)$, unreachable $(n=4)$ and other reasons $(n=5)$.

The mean follow-up period was 9.06 year $(S D=0.14$ ). Included patients $(n=90)$ did not differ from excluded patients ( $n=128)$ in age $(51.2 \pm 11.9$ versus $52.3 \pm 13.1$ years, respectively, $p=0.498$ ) or sex (male $57.8 \%$ versus $45.3 \%$, respectively, $p=0.070$ ). There was a difference in presence of periventricular WMH at baseline between included patients and excluded patients (26.2\% versus 44.9\%, respectively, $p=0.027$ ), but there was no difference in presence of any of the other cSVD markers at baseline between included and excluded patients (all p>0.05). Baseline characteristics are shown in table 1. Brain MRI of one patient was of insufficient quality to determine progression of $\mathrm{WMH}$; therefore analyses for progression of $\mathrm{WMH}$ included 89 patients.

\section{Progression of cSVD markers on MRI}

In simple regression analyses, FSRP was associated with progression of periventricular WMH and with new microbleeds (table 2). These results did not change after correction for baseline presence of the MRI marker. In both simple and multivariable regression anal- 
yses, no significant associations were found between FSRP and progression of subcortical WMH and new lacunes (table 2).

Table 1. Patients' characteristics

\begin{tabular}{ll}
\hline & All patients $(n=90)$ \\
\hline Baseline assessment & $51.2(11.9)$ \\
Age, mean (SD), years & $52(57.8)$ \\
Male sex, No. (\%) & $160.0(148.75-180.5)$ \\
Systolic blood pressure, mmHg, median (IQR) & $24(26.7)$ \\
History of cardiovascular disease, No (\%) & $6(6.7)$ \\
Left ventricular hypertrophy, No. (\%) & $14(15.6)$ \\
Smoking, No. (\%) & $8.5(6.0-13.0)$ \\
FSRP total score, median (IQR) & \\
Follow-up assessment & $86(74-94)$ \\
DART, median (IQR) & $6(4-12)$ \\
HADS total score, median (IQR) & $23(25.8)$ \\
Progression of periventricular WMH, No. (\%)* & $44(49.4)$ \\
Progression of subcortical WMH, No. (\%)* & $7(7.8)$ \\
New microbleeds, No. (\%) & $9(10.0)$ \\
New lacunes, No. (\%) & \\
\hline
\end{tabular}

$S D=$ standard deviation; $I Q R=$ interquartile range; FSRP=Framingham stroke risk profile; DART=Dutch adult reading test; HADS=hospital anxiety and depression scale; WMH=white matter hyperintensities

* Information about progression of periventricular and subcortical WMH was missing for 1 patient

Table 2. Associations between FSRP and progression of MRI markers

\begin{tabular}{|c|c|c|c|c|c|c|c|c|}
\hline & \multicolumn{2}{|c|}{ Periventricular WMH } & \multicolumn{2}{|c|}{ Subcortical WMH } & \multicolumn{2}{|l|}{ Lacunes } & \multicolumn{2}{|l|}{ Microbleeds } \\
\hline & OR $(95 \% \mathrm{Cl})$ & $p$ & $O R(95 \% \mathrm{CI})$ & $p$ & OR $(95 \% \mathrm{Cl})$ & $p$ & OR $(95 \% \mathrm{Cl})$ & $p$ \\
\hline $\begin{array}{l}\text { Unadjusted logistic } \\
\text { regression }\end{array}$ & $\begin{array}{l}1.19 \\
(1.05-1.36)\end{array}$ & 0.008 & $\begin{array}{l}1.07 \\
(0.96-1.20)\end{array}$ & 0.196 & $\begin{array}{l}1.08 \\
(0.91-1.29)\end{array}$ & 0.368 & $\begin{array}{l}1.31 \\
(1.04-1.64)\end{array}$ & 0.022 \\
\hline $\begin{array}{l}\text { Corrected for baseline } \\
\text { MRI marker }\end{array}$ & $\begin{array}{l}1.18 \\
(1.03-1.36)\end{array}$ & 0.017 & $\begin{array}{l}1.04 \\
(0.92-1.16)\end{array}$ & 0.549 & $\begin{array}{l}1.02 \\
(0.85-1.23)\end{array}$ & 0.840 & $\begin{array}{l}1.29 \\
(1.02-1.64)\end{array}$ & 0.031 \\
\hline
\end{tabular}

FSRP=Framingham stroke risk profile; $\mathrm{WMH}=$ white matter hyperintensities; $O R=$ odds ratio; $\mathrm{Cl}=$ confidence interval

\section{Cognitive performance}

At follow-up, 3 patients had an R-CAMCOG score <34, indicating possible dementia.

Simple linear regression analyses showed that higher FSRP was associated with lower cognitive performance in overall cognition, executive function, information processing speed and memory (table 3). Correction for DART and HADS score did not change the results (table 3 ). 
Table 3. Associations between FSRP and cognitive performance

\begin{tabular}{lllllllll}
\hline & Overall cognition & \multicolumn{2}{l}{ Executive function } & \multicolumn{2}{l}{$\begin{array}{l}\text { Information } \\
\text { processing speed }\end{array}$} & \multicolumn{2}{l}{ Memory } \\
& $B(95 \% \mathrm{Cl})$ & $p$ & $B(95 \% \mathrm{Cl})$ & $p$ & $B(95 \% \mathrm{Cl})$ & $p$ & $B(95 \% \mathrm{Cl})$ & $p$ \\
\hline Model 1 & -0.10 & $<0.001$ & -0.07 & $<0.001$ & -0.12 & $<0.001$ & -0.09 & $<0.001$ \\
& $(-0.13$ to -0.06$)$ & & $(-0.11$ to -0.03$)$ & & $(-0.16$ to -0.09$)$ & & $(-0.12$ to -0.06$)$ & \\
Model 2 & -0.09 & $<0.001$ & -0.07 & 0.001 & -0.12 & $<0.001$ & -0.09 & $<0.001$ \\
& $(-0.12$ to-0.06) & & $(-0.10$ to -0.03$)$ & & $(-0.16$ to -0.09$)$ & & $(-0.12$ to -0.06$)$ & \\
\hline
\end{tabular}

FSRP=Framingham stroke risk profile; DART=Dutch adult reading test; HADS=hospital anxiety and depression scale; $B=$ unstandardized regression coefficient; $\mathrm{Cl}=$ confidence interval

Model 1: unadjusted linear regression

Model 2: corrected for DART \& HADS

\section{Exploratory analyses}

The association between FSRP and progression of periventricular $\mathrm{WMH}$, corrected for baseline presence of periventricular WMH, lost significance after additional adjustment for the FSRP age component, but remained significant with correction for the FSRP SBP component. The association between FSRP and new microbleeds was no longer significant after adjustment for either the component of age or SBP.

The association between FSRP and cognitive performance in overall cognition, information processing speed and memory, corrected for DART and HADS, remained significant after additional correction for the age or SBP component. Only for executive function, the association with FSRP lost significance after adjustment for age. All associations between FSRP and cognitive performance remained significant after additional correction for baseline total burden of CSVD.

\section{DISCUSSION}

The results of the present study show that the Framingham stroke risk profile is associated with progression of periventricular WMH and with new microbleeds over 9 years of follow-up in patients with essential hypertension. We could not show associations between FSRP and progression of subcortical WMH and new lacunes. Furthermore, we showed that FSRP was associated with cognitive performance.

Previous studies showed that the FSRP is associated with presence of MRI markers of CSVD, but longitudinal results were lacking. We performed a longitudinal study into the association between FSRP and progression of CSVD. Our finding that FSRP is associated with progression of periventricular WMH but not with progression of subcortical WMH 
is in agreement with an earlier cross-sectional study. ${ }^{12}$ Another study showed that FSRP was associated with total $\mathrm{WMH}$, but did not differentiate between periventricular and subcortical WMH. ${ }^{11}$ Even though baseline presence of WMH is a strong predictor of its progression, ${ }^{36}$ we showed that the association between FSRP and progression of periventricular WMH was independent of baseline presence of periventricular $\mathrm{WMH}$.

We also showed an association between FSRP and new microbleeds. Only one earlier cross-sectional study investigated the association between FSRP and microbleeds and showed that FSRP was higher in patients with microbleeds compared to patients without microbleeds. ${ }^{15}$ During our 9-year follow-up study, only a few patients developed new lacunes, which is quite low compared to other studies, ${ }^{37}$ and we could not show an association between FSRP and new lacunes. Larger studies into the association between FSRP and new microbleeds and lacunes are needed.

We found that FSRP is associated with cognitive performance, which is in agreement with previous studies in other populations. In a cross-sectional study in the Framingham Offspring Cohort, Elias et al. ${ }^{16}$ showed that the FSRP was associated with lower cognitive performance in several cognitive domains. Another cohort study showed that the FSRP in midlife was associated with cognitive decline over 10 years. ${ }^{17}$ From our study, we can conclude that these results are similar in hypertensive patients, who have a higher risk for CSVD and cognitive deficits.

Age is generally considered the most important risk factor for cSVD and cognitive decline. In addition to age, hypertension is a major risk factor for CSVD. Therefore, the associations of FSRP with progressive CSVD and cognitive performance might be attributable to only age or SBP. Since our exploratory analyses showed that the association between FSRP and periventricular WMH lost significance after adjustment for the FSRP age component, this confirms the idea that age is probably the most important factor of the FSRP in the association with progression of periventricular WMH. For new microbleeds, the association with FSRP lost significance after adjustment for either age or SBP. For overall cognition, the association with FSRP remained significant after adjustment for any of the FSRP components. Therefore, we can conclude that the association between FSRP and cognitive performance is not explained by only age or SBP, but that multiple components are implicated in the association.

A major strength of our study is the longitudinal design with a 9-year follow-up with brain MRI scans at baseline and at follow-up to determine progression of markers. In addition, patients received an extensive neuropsychological assessment with multiple tests in three different cognitive domains. A limitation of the study is the lack of neuropsychological 
assessment at baseline, which withheld us from determining cognitive decline over these 9 years. However, since we used the DART to control for prior cognitive performance, results actually reflect associations with a lifetime change in cognitive performance. Secondly, although the long follow-up time is a strength of our study, this was coupled with a high drop-out rate. Only 90 patients of the 218 patients included at baseline responded to the follow-up and this might have caused a selection bias. Patients excluded from follow-up might be those with worse cognitive function. Thirdly, since diabetes mellitus and atrial fibrillation were exclusion criteria for our study, not the entire spectrum of risk factors in the FSRP is present in our sample. This might also cause the remaining factors, especially age and hypertension, to be more important in the FSRP in our cohort and limits the generalizability of the results.

In conclusion, our results show that the Framingham stroke risk profile, a composite vascular risk score originally developed to predict symptomatic stroke, is also associated with more 'silent' effects of cerebrovascular disease, such as progressive cSVD and cognitive performance, in patients with hypertension. While we showed that the age component was the most important for progressive CSVD, this was not the case for cognitive performance. Our results support the importance of early detection of other vascular risk factors in patients with hypertension. Clinicians should be aware of the entire risk factor profile in attempting to preserve cognitive performance. Future studies are needed to examine if the predictive value of the risk factor profile could be improved by including more factors, such as education or prior cognitive ability. 


\section{REFERENCES}

1. Pantoni L. Cerebral small vessel disease: from pathogenesis and clinical characteristics to therapeutic challenges. Lancet Neurol. 2010;9:689-701.

2. Prins ND, Scheltens P. White matter hyperintensities, cognitive impairment and dementia: an update. Nat Rev Neurol. 2015;11:157-165.

3. Wallin A, Öhrfelt A, Bjerke M. Characteristic clinical presentation and CSF biomarker pattern in cerebral small vessel disease. J Neurol Sci. 2012;322:192-196.

4. Veglio F, Paglieri C, Rabbia F, Bisbocci D, Bergui M, Cerrato P. Hypertension and cerebrovascular damage. Atherosclerosis. 2009;205:331-341.

5. Gouw AA, van der Flier WM, Fazekas F, van Straaten EC, Pantoni L, Poggesi A, Inzitari D, et al. Progression of white matter hyperintensities and incidence of new lacunes over a 3-year period: The Leukoaraiosis and Disability Study. Stroke. 2008;39:1414-1420.

6. Wardlaw JM, Smith C, Dichgans M. Mechanisms of sporadic cerebral small vessel disease: insights from neuroimaging. Lancet Neurol. 2013;12:483-497.

7. Vermeer SE, Longstreth WT, Koudstaal PJ. Silent brain infarcts: a systematic review. Lancet Neurol. 2007;6:611-619.

8. Nash DT, Fillit H. Cardiovascular disease risk factors and cognitive impairment. Am J Cardiol. 2006;97:12621265.

9. North T-L, Palmer TM, Lewis SJ, Cooper R, Power C, Pattie A, Starr JM, et al. Effect of smoking on physical and cognitive capability in later life: a multicohort study using observational and genetic approaches. BMJ open. 2015;5:e008393.

10. Baumgart M, Snyder HM, Carrillo MC, Fazio S, Kim H, Johns H. Summary of the evidence on modifiable risk factors for cognitive decline and dementia: A population-based perspective. Alzheimers Dement. 2015;11:718-726.

11. Jeerakathil T, Wolf PA, Beiser A, Massaro J, Seshadri S, D'Agostino RB, DeCarli C. Stroke risk profile predicts white matter hyperintensity volume - The Framingham study. Stroke. 2004;35:1857-1861.

12. Smith PJ, Blumenthal JA, Babyak MA, Watkins LL, Hinderliter A, Hoffman BM, Steffens DC, et al. Cerebrovascular risk factors and cerebral hyperintensities among middle-aged and older adults with major depression. Am J Geriatr Psychiatry. 2010;18:848-852.

13. Delgado P, Riba-Llena I, Tovar JL, Jarca Cl, Mundet X, López-Rueda A, Orfila F, et al. Prevalence and associated factors of silent brain infarcts in a Mediterranean cohort of hypertensives. Hypertension. 2014;64:658-663.

14. Das RR, Seshadri S, Beiser AS, Kelly-Hayes M, Au R, Himali JJ, Kase CS, et al. Prevalence and correlates of silent cerebral infarcts in the Framingham offspring study. Stroke. 2008;39:2929-2935.

15. Ochi N, Tabara Y, Igase M, Nagai T, Kido T, Miki T, Kohara K. Silent cerebral microbleeds associated with arterial stiffness in an apparently healthy subject. Hypertens Res. 2009;32:255-260.

16. Elias MF, Sullivan LM, D'Agostino RB, Elias PK, Beiser A, Au R, Seshadri S, et al. Framingham stroke risk profile and lowered cognitive performance. Stroke. 2004;35:404-409.

17. Kaffashian S, Dugravot A, Brunner EJ, Sabia S, Ankri J, Kivimäki M, Singh-Manoux A. Midlife stroke risk and cognitive decline: a 10-year follow-up of the Whitehall II cohort study. Alzheimers Dement. 2013;9:572-579.

18. Henskens LH, van Oostenbrugge RJ, Kroon AA, de Leeuw PW, Lodder J. Brain microbleeds are associated with 
ambulatory blood pressure levels in a hypertensive population. Hypertension. 2008;51:62-68.

19. D'Agostino RB, Wolf PA, Belanger AJ, Kannel WB. Stroke risk profile: adjustment for antihypertensive medication. The Framingham Study. Stroke. 1994;25:40-43.

20. Wolf PA, D'Agostino RB, Belanger AJ, Kannel WB. Probability of stroke: a risk profile from the Framingham Study. Stroke. 1991;22:312-318.

21. Uiterwijk R, Huijts M, Staals J, Duits A, Gronenschild E, Kroon AA, de Leeuw PW, et al. Subjective cognitive failures in patients with hypertension are related to cognitive performance and cerebral microbleeds. Hypertension. 2014;64:653-657.

22. Brand N, Jolles J. Learning and retrieval rate of words presented auditorily and visually. J Gen Psychol. 1985;112:201-210.

23. Wechsler D. WAIS III, nederlandstalige bewerking: technische handleiding. Lisse: Swets Test Publishers; 2001.

24. Golden CJ. Stroop Colour and Word Test. Chicago (IL): Stoelting; 1978.

25. Reitan R. Trail Making Test: Manual for administration, scoring and interpretation. Bloomington: Indiana University; 1956.

26. Luteyn F. Een nieuwe verkorte GIT. Dutch J Psychol. 1966;2:675-682.

27. Lezak MD, Howieson DB, Loring DW. Neuropsychological assessment. New York: Oxford University Press; 2004.

28. Schmand B, Bakker D, Saan R, Louman J. The Dutch Reading Test for Adults: a measure of premorbid intelligence level. Tijdschr Gerontol Geriatr. 1991;22:15-19.

29. De Koning I, van Kooten F, Koudstaal PJ, Dippel DW. Diagnostic value of the Rotterdam-CAMCOG in post-stroke dementia. J Neurol Neurosurg Psychiatry. 2005;76:263-265.

30. Spinhoven PH, Ormel J, Sloekers PP, Kempen GlJ, Speckens AE, van Hemert AM. A validation study of the Hospital Anxiety and Depression Scale (HADS) in different groups of Dutch subjects. Psycho/ Med. 1997;27:363-370.

31. Huijts M, Duits A, van Oostenbrugge RJ, Kroon AA, de Leeuw PW, Staals J. Accumulation of MRI markers of cerebral small vessel disease is associated with decreased cognitive function: A study in first-ever lacunar stroke and hypertensive patients. Front Aging Neurosci. 2013;5:72

32. Fazekas F, Chawluk JB, Alavi A, Hurtig HI, Zimmerman RA. MR signal abnormalities at $1.5 \mathrm{~T}$ in Alzheimer's dementia and normal aging. AJR Am J Roentgenol. 1987;149:351-356.

33. Wardlaw JM, Smith EE, Biessels GJ, Cordonnier C, Fazekas F, Frayne R, Lindley Rl, et al. Neuroimaging standards for research into small vessel disease and its contribution to ageing and neurodegeneration. Lancet Neurol. 2013;12:822-838

34. Uiterwijk R, van Oostenbrugge RJ, Huijts M, de Leeuw PW, Kroon AA, Staals J. Total cerebral small vessel disease MRI score is associated with cognitive decline in executive function in patients with hypertension. Front Aging Neurosci. 2016;8.

35. Prins N, van Straaten E, van Dijk E, Simoni M, van Schijndel R, Vrooman H, Koudstaal P, et al. Measuring progression of cerebral white matter lesions on MRI Visual rating and volumetrics. Neurology. 2004;62:1533-1539.

36. Schmidt R, Enzinger C, Ropele S, Schmidt H, Fazekas F. Progression of cerebral white matter lesions: 6-year results of the Austrian Stroke Prevention Study. The Lancet. 2003;361:2046-2048.

37. Schmidt R, Seiler S, Loitfelder M. Longitudinal change of small-vessel disease-related brain abnormalities. J Cereb Blood Flow Metab. 2016;36:26-39. 




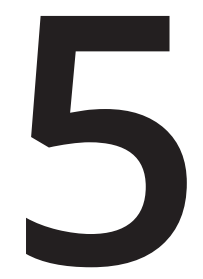

Hypertensive organ damage predicts future cognitive performance: A 9-year follow-up study in patients with hypertension

Uiterwijk R, Staals J, Huijts M, van Kuijk SMJ, de Leeuw PW, Kroon AA, van Oostenbrugge RJ 


\begin{abstract}
Objectives Hypertension is associated with cognitive deficits, probably caused by brain damage resulting from hypertension-related cerebral Small Vessel Disease (cSVD). Hypertension is also related to other organ damages, namely cardiac and renal damage. We examined if these types of organ damage and their combined presence are associated with future lower cognitive performance.
\end{abstract}

Methods In patients with essential hypertension, brain damage was determined by MRI features summed in the cSVD burden score. Cardiac damage was determined by echocardiography-based left ventricular mass index (LVMI). Renal damage was determined by estimated Glomerular Filtration Rate (eGFR) and albuminuria. At 9-year follow-up, an extensive neuropsychological assessment was performed. With linear regression analyses, we tested 1) if increasing number of organ damages (brain, cardiac and/or renal damage) was associated with cognitive performance and 2) whether LVMI, eGFR and albuminuria were associated with cognition, independent of age, sex, premorbid cognition and cSVD score.

Results We included 78 patients with hypertension (mean age 51.2 \pm 12.0 years), who completed follow-up. Presence of 2 or 3 types of organ damage compared to none was associated with future lower cognitive performance ( $p=0.023$ and $p=0.012$, respectively). In addition, LVMI was independently associated with future lower cognition ( $p=0.032$ ), but eGFR and albuminuria were not ( $p=0.624$ and $p=0.714$, respectively).

Conclusion Increasing number of sites with hypertensive organ damage, and especially cardiac damage independently of brain damage, is associated with future lower cognitive performance. This might indicate a more severe hypertensive disease burden and could help to identify patients at high risk of developing cognitive problems. 


\section{INTRODUCTION}

The brain is one of the target organs affected by hypertension. ${ }^{1}$ Hypertension is a major risk factor for cerebral Small Vessel Disease (CSVD), ${ }^{2}$ which in turn leads to brain damage which can be made visible on brain MRI. Such imaging markers of CSVD are strongly associated with cognitive function. . $^{3-6}$ Other types of hypertensive organ damage, such as cardiac and renal damage, have also been linked to lower cognitive function or increased risk of cognitive impairment. ${ }^{7-11}$

Organ damage could be regarded as marker of the severity of hypertensive disease. ${ }^{12}$ Little research has investigated the combined effect of different sites of organ damage on cognitive function. One study showed that an increasing number of signs of cardiac and renal damage was associated with decline in memory performance. ${ }^{13}$ However, whether cardiac and renal damage are also associated with cognition independently of brain damage is unknown. Investigating the implications of combined and organ-specific hypertensive organ damage could help identifying patients at high risk of developing cognitive problems.

We investigated whether brain, cardiac and renal damage, and their combined presence, are predictive of lower cognitive performance 9 years later. In addition, we examined if cardiac and renal hypertensive organ damage are predictive of future lower cognitive performance, independent of CSVD, and what these types of hypertensive organ damage add, in addition to CSVD, in explaining cognitive function.

\section{METHODS}

\section{Study population}

Patients with essential hypertension were recruited from the hypertension outpatient clinic of the Department of Internal Medicine of Maastricht University Medical Centre, the Netherlands, for a study on brain damage in patients with essential hypertension (HYBRiD). ${ }^{14}$ Hypertension was defined as off-medication, clinically measured conventional blood pressure $\geq 140 \mathrm{~mm}$ Hg systolic or $\geq 90 \mathrm{~mm}$ Hg diastolic, or both. Details about the HYBRiD study have been described before. ${ }^{14}$ Exclusion criteria were documented diabetes, ischemic or valvular heart disease, atrial fibrillation, history of transient ischemic attacks or stroke and obstructive sleep apnea syndrome. All patients received a brain MRI, an echocardiography and a timed urine collection over a 24-hour period and routine laboratory investigations. Three off-medication office blood pressure measurements were taken and the systolic blood pressure (SBP), diastolic blood pressure (DBP) and mean arterial pressure (MAP) from the second and third measurement were averaged. Nine 
years later, patients were invited for follow-up study, which included a neuropsychological assessment. The Medical Ethics Committee of the Maastricht University Medical Centre approved this study and all participants gave written informed consent.

\section{Assessment of brain damage}

On brain MRI scans (axial T2-weighted, FLAIR and T2* gradient echo sequences, Intera 1.5-T, Philips Medical Systems, Best, The Netherlands) two experienced vascular neurologists rated MRI markers of CSVD, after reaching satisfactory inter-rater agreements. The inter-rater agreement statistics have been previously reported. ${ }^{15}$ An ordinal scale representing the total burden of CSVD was created. Definitions and rating method have been described in detail before. ${ }^{16}$ In short, the presence of each of the four MRI markers for CSVD (white matter hyperintensities (WMH), lacunes, cerebral microbleeds and perivascular spaces) was counted to retrieve a total CSVD burden score (ranging 0-4). WMH were assessed according to the Fazekas scale. ${ }^{17}$ Presence of WMH was defined as periventricular WMH Fazekas score 3 (irregular hyperintensities extending into the deep white matter) and/or deep WMH Fazekas score 2 or 3 (confluent hyperintensities). Perivascular spaces were rated at the level of the basal ganglia as mild, moderate or extensive. One point was awarded for moderate or extensive perivascular spaces. Lacunes and cerebral microbleeds were assessed according to the international consensus definition ${ }^{18}$ and for both lacunes and cerebral microbleeds, one point was awarded in the total cSVD score if at least one lacune or cerebral microbleed was present. Brain damage was considered present in case of a cSVD score $\geq 1$.

\section{Assessment of cardiac damage}

Left ventricular mass, assessed by two-dimensional M-mode and Doppler echocardiography (Sonos 5500, Hewlett-Packard, Andover, MA) was estimated according to Devereux's formula (Penn convention) and indexed to body surface area. The left ventricle dimensions and wall thicknesses were measured according to recommendations. ${ }^{19}$ Cardiac damage was considered present in case of left ventricular hypertrophy (LVH); defined as a left ventricular mass index $(\mathrm{LVMI})>115 \mathrm{~g} / \mathrm{m}^{2}$ for men and >95 g/m² for women.

\section{Assessment of renal damage}

The urinary albumin excretion (UAE) was measured in a timed 24-hour urine collection by means of nephelometry (BN ProSpec ${ }^{\circledR}$ System, Dade Behring Inc.), with a detection limit of $2.2 \mathrm{mg} / \mathrm{l}$. Microalbuminuria was defined as a UAE >30 and <300 mg/24h. Renal function was considered to be impaired in case of an estimated Glomerular Filtration Rate (eGFR; calculated with CKD-EPI) $<60 \mathrm{ml} / \mathrm{min}$. Hypertension-related renal damage was considered to be present in case of microalbuminuria and/or impaired renal function. 
Results of reproducibility analyses for both cardiac and renal damage have been described before. $^{12}$

\section{Neuropsychological assessment}

Cognitive performance was measured at 9 year follow-up with an extensive neuropsychological assessment; as has been described before. ${ }^{20}$ Memory domain was measured with the Rey Auditory Verbal Learning Test ${ }^{21}$ (immediate recall, delayed recall and delayed recognition) and the Digit Span Forward (subtest of Wechsler Adult Intelligence Scale (WAIS)-III22). Executive function domain was measured with the Stroop Colour Word Test ${ }^{23}$ (SCWT) interference score (time of part 3 minus mean time of parts 1 and 2), Trail Making Test $^{24}$ (TMT) interference score (time of part 2 minus time of part 1), Category (animals and professions) ${ }^{25}$ and Letter Fluency ${ }^{26}$, Letter-Number Sequencing (subtest of WAIS-III), and Digit Span Backward (subtest of WAIS-III). Information processing speed domain was measured with the Symbol Substitution - Coding (subtest of WAIS-III), TMT part A, and SCWT parts 1 and 2. Test scores were transformed into standardized values (z-scores), by dividing the difference between the individual raw score and the sample mean by the sample standard deviation. For each patient, domain scores were calculated by averaging these z-scores of the tests within that domain. Finally, the overall cognition score was calculated by averaging the domain scores of memory, executive function and information processing speed.

The Dutch Adult Reading Test (DART) was used as a measure of prior cognitive ability. ${ }^{27}$ By correcting for the DART, associations with cognitive function actually represent a lifetime change in cognitive performance.

\section{Statistical analysis}

Baseline characteristics of all included patients were reported as mean and standard deviation (SD) or median and interquartile range (IQR) for normally and non-normally distributed characteristics, respectively, and absolute number and percentage for categorical characteristics.

We investigated whether an increasing number of sites with organ damage (brain, cardiac and/or renal damage) were associated with future cognitive performance using multivariable linear regression analysis, corrected for age, sex, and DART score. Next, we tested the associations between the individual organ damages (cSVD score, LVMI, eGFR and UAE; as continuous variables) and cognitive performance. We performed univariable linear regression analyses to estimate crude associations, and subsequently performed multivariable linear regression in which we corrected for age, sex, and DART score. In addition, for the analyses of LVMI, eGFR and UAE, we repeated the multivariable linear regression with 
addition of the cSVD score. For all regression analyses, we computed the coefficient of variation $\left(R^{2}\right)$, to examine how much of the variance in cognitive performance was explained by LVMI, eGFR or UAE, on top of age, sex, DART score and CSVD score.

In exploratory analyses, we examined the associations between SBP, DBP and MAP and cSVD score, LVMI, eGFR and UAE with Pearson correlation, and the number of sites with organ damage with Spearman's correlation. IBM SPSS Statistics 22 software was used for all analyses. Results were considered significant at $p<0.05$.

\section{RESULTS}

\section{Participants}

Of the 218 patients in the HYBRiD study at baseline, 193 (88.5\%) patients had complete baseline data on brain, cardiac and renal damage. Of these, 78 (40.4\%) completed the follow-up including cognitive testing after 9 years. Reasons for exclusion during follow-up were: no consent to be contacted for follow-up ( $n=18$ ), not interested ( $n=70)$, cerebrovascular event during follow-up $(n=7)$, death $(n=4)$, contra-indications for MRI $(n=4)$, neuropsychological assessment not possible $(n=3)$, unreachable $(n=3)$ and other reasons $(n=6)$.

The mean follow-up period was 9.05 year $(S D=0.13)$. Included patients ( $n=78)$ did not differ from excluded patients ( $n=115)$ in age $(51.2 \pm 12.0$ versus $52.0 \pm 12.7$ years, respectively, $p=0.670$ ), sex (male $56.4 \%$ versus $47.0 \%$, respectively, $p=0.197)$, LVMI ( $p=0.544)$, eGFR $(p=0.843)$ or $\cup A E(p=0.254)$. Baseline characteristics are shown in table 1.

\section{Hypertensive organ damage}

Twenty-nine patients had no organ damage, 31 had damage of 1 organ, 15 had 2 organ damages and 3 had damage of all 3 organs. The distribution of the types of organ damage is shown in table 2 .

Presence of 2 or 3 sites with organ damage was associated with future lower cognitive performance $(B=-0.36(95 \% \mathrm{Cl}=-0.67$ to -0.05$), p=0.023$, and $B=-0.77(95 \% \mathrm{Cl}=-1.37$ to -0.18$)$, $p=0.012$, respectively) compared to having no organ damage, but having organ damage only at one site was not ( $B=-0.16(95 \% \mathrm{Cl}=-0.42$ to 0.11$), p=0.238)$.

CSVD score was associated with lower cognitive performance in unadjusted and adjusted analyses (table 3). LVMI was associated with lower cognition, in unadjusted analysis and after correction for age, sex and DART score and additionally for cSVD score (table 3). eGFR and UAE were both not associated with cognitive performance (table 3). 
Table 1. Patients' characteristics

\begin{tabular}{ll}
\hline & All patients $(n=78)$ \\
\hline Age, mean (SD), years & $51.2(12.0)$ \\
Male sex, No. (\%) & $44(56.4)$ \\
Systolic blood pressure, mmHg, median (IQR) & $161(150-181)$ \\
Diastolic blood pressure, mmHg, mean (SD) & $101(11)$ \\
Mean arterial pressure, mmHg, mean (SD) & $123(14)$ \\
Duration of hypertension, months, median (IQR) & $38(14-126)$ \\
DART score, median (/QR) & $85(74-92.5)$ \\
LVMI, mean (SD), g/m² & $88(18)$ \\
eGFR, mean (SD), ml/min/1.73m² & $83(17)$ \\
UAE, median (IQR), mg/24h & $12(7-20)$ \\
CSVD score 0, No. (\%) & $39(50 \%)$ \\
CSVD score 1, No. (\%) & $28(35.9 \%)$ \\
CSVD score 2, No. (\%) & $5(6.4 \%)$ \\
CSVD score 3, No. (\%) & $5(6.4 \%)$ \\
CSVD score 4, No. (\%) & $1(1.3 \%)$ \\
\hline
\end{tabular}

$S D=$ standard deviation; IQR=interquartile range; DART=Dutch adult reading test; $L V M I=$ left ventricular mass index;

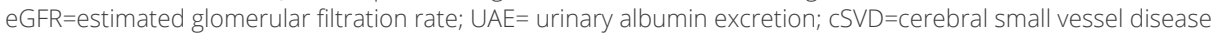

Table 2. Distribution and number of sites with organ damages

\begin{tabular}{lllll}
\hline $\begin{array}{l}\text { Number of organ damages } \\
\text { Number of patients (\%) }\end{array}$ & 0 & 1 & 2 & 3 \\
\hline Number of patients with: & $n=29(37.2 \%)$ & $n=31(39.7 \%)$ & $n=15(19.2 \%)$ & $n=3(3.8 \%)$ \\
\hline Presence of brain damage & $0(0 \%)$ & $21(67.7 \%)$ & $15(100 \%)$ & $3(100 \%)$ \\
Presence of cardiac damage & $0(0 \%)$ & $5(16.1 \%)$ & $6(40.0 \%)$ & $3(100 \%)$ \\
Presence of renal damage & $0(0 \%)$ & $5(16.1 \%)$ & $9(60.0 \%)$ & $3(100 \%)$ \\
\hline
\end{tabular}

In addition, we investigated how much variance in future cognitive performance was explained by hypertensive organ damage. Age, sex and DART score explained $44.7 \%$ of the variance in cognitive performance. The CSVD score explained an additional 5.2\%. On top of age, sex, DART score and CSVD score, 3.1\% was explained by LVMI, while eGFR and UAE explained only $0.2 \%$ and $0.1 \%$, respectively.

\section{Exploratory analyses}

Since types of organ damage are thought to result from hypertension, we examined the correlations between SBP, DBP and MAP and organ damages. Results are shown in table 4. 
Table 3. Associations between organ damage and cognitive performance

\begin{tabular}{|c|c|c|c|c|}
\hline & cSVD score & & LVMI & \\
\hline & $B(95 \% \mathrm{Cl})$ & $p$ & $B(95 \% \mathrm{Cl})$ & $p$ \\
\hline Unadjusted linear regression & $-0.237(-0.390$ to -0.084$)$ & 0.003 & $-0.009(-0.017$ to -0.001$)$ & 0.024 \\
\hline Corrected for age, sex, DART score & $-0.173(-0.298$ to -0.048$)$ & 0.007 & $-0.008(-0.014$ to -0.002$)$ & 0.010 \\
\hline $\begin{array}{l}\text { Corrected for age, sex, DART score } \\
\text { and CSVD score }\end{array}$ & NA & NA & $-0.007(-0.013$ to -0.001$)$ & 0.032 \\
\hline \multicolumn{5}{|l|}{ Table 3 continued } \\
\hline & eGFR & & UAE & \\
\hline & $B(95 \% \mathrm{Cl})$ & $p$ & $B(95 \% \mathrm{Cl})$ & $p$ \\
\hline Unadjusted linear regression & $0.008(-0.001$ to 0.017$)$ & 0.077 & $-0.001(-0.005$ to 0.003$)$ & 0.574 \\
\hline Corrected for age, sex, DART score & $-0.001(-0.009$ to 0.007$)$ & 0.881 & $-0.002(-0.004$ to 0.001$)$ & 0.232 \\
\hline $\begin{array}{l}\text { Corrected for age, sex, DART score } \\
\text { and cSVD score }\end{array}$ & $-0.002(-0.010$ to 0.006$)$ & 0.624 & $-0.001(-0.003$ to 0.002$)$ & 0.714 \\
\hline
\end{tabular}

CSVD=cerebral small vessel disease; LVMI=left ventricular mass index; eGFR=estimated glomerular filtration rate; $\mathrm{UAE}=$ urinary albumin excretion; DART=Dutch adult reading test; $B=$ unstandardized regression coefficient; $\mathrm{Cl}=\mathrm{Con}$ fidence interval; NA=not applicable

Table 4. Correlations between blood pressure levels and organ damage

\begin{tabular}{llllll}
\hline & cSVD score & LVMI & eGFR & UAE & $\begin{array}{l}\text { Number of organ } \\
\text { damages }\end{array}$ \\
\hline SBP & $r=0.294, p=0.009$ & $r=0.406, p<0.001$ & $r=-0.248, p=0.029$ & $r=0.388, p<0.001$ & $\rho=0.286, p=0.011$ \\
DBP & $r=0.194, p=0.089$ & $r=0.319, p=0.004$ & $r=0.034, p=0.767$ & $r=0.334, p=0.003$ & $\rho=0.276, p=0.014$ \\
MAP & $r=0.273, p=0.016$ & $r=0.405, p<0.001$ & $r=-0.119, p=0.298$ & $r=0.403, p<0.001$ & $\rho=0.326, p=0.004$ \\
\hline
\end{tabular}

CSVD=cerebral small vessel disease; LVMI=left ventricular mass index; eGFR=estimated glomerular filtration rate; $\mathrm{UAE}=$ urinary albumin excretion; $\mathrm{SBP}=$ systolic blood pressure; $\mathrm{DBP}=$ diastolic blood pressure; $\mathrm{MAP}=$ mean arterial pressure; $r=$ Pearson correlation coefficient; $\rho=$ Spearman's correlation coefficient

\section{DISCUSSION}

We showed that an increasing number of sites with hypertensive organ damage (brain, cardiac, and/or renal damage) was associated with lower cognitive performance after 9 years of follow-up. In addition, our results showed that cardiac damage is associated with cognitive performance after 9 year, independently of brain MRI damage.

The mechanisms underlying hypertension-related cognitive changes are not fully known, but are thought to be mainly induced by CSVD. CSVD is represented by visible MRI brain damage, but is also associated with microstructural brain damage and cerebrovascular functional alterations such as hypoperfusion. These factors also relate to cognitive function and thus, CSVD score determined by standard brain MRI only partly represents the 
impact of hypertension and CSVD on cognitive function. Therefore, we examined whether additional clinical effect markers of hypertension, namely other types of organ damage, could predict future cognitive dysfunction, on top of visible MRI brain damage.

Not surprisingly, cSVD score predicted the largest proportion of variance in cognitive performance, but cardiac damage was also independently associated with cognitive performance. Our results are in agreement with previous studies that showed associations between cardiac damage and cognitive function., However, as these studies did not investigate this association independently of brain damage, these results might be due to a high correlation between hypertensive cardiac damage and brain damage.

The result that an increasing number of sites with hypertensive organ damage is predictive of lower cognitive performance, underlines the importance of considering total hypertensive organ damage instead of only determining damage in one or two organs. Previous results showed that there is only limited overlap between different sites of hypertensive organ damage, as organ damage can co-occur, but can also remain restricted to one organ. ${ }^{12}$ We are aware of one other study that investigated the implications of combined hypertensive organ damages. ${ }^{13}$ In this study, number of organ damages was based on $\mathrm{LVH}$, impaired renal function and albuminuria. It was shown that presence of 2 or 3 organ damages was associated with decline in memory performance (but not executive function), compared to having no organ damage.

The focus of our hypothesis was the association between the presence of hypertensive organ damage and cognitive dysfunction 9 years later. Hypertension causes organ damage, which in turn, affects cognitive function. We did not correct for blood pressure in our analyses since the different forms of organ damage are intermediate variables in the causal pathway between hypertension and cognitive dysfunction. In our cohort, all 3 types of organ damage were indeed correlated with SBP, DBP and/or MAP. The number of sites with organ damage was also correlated with blood pressure.

An important strength of the study is the extensive neuropsychological assessment. The long follow-up time of 9 years is also a strength of our study, but this was also coupled with a high drop out rate, as of 193 patients included at baseline only 78 also completed follow-up. It is possible that cognitively worse patients were less likely to participate in the follow-up measurements. Another limitation is the lack of neuropsychological assessment at baseline, which withheld us from determining cognitive decline over the 9 years of follow-up. However, since we used the DART score to control for prior cognitive performance, results actually reflect associations with a lifetime change in cognitive performance. 
In conclusion, the load of organ damage, i.e. having an increasing number of sites with hypertensive organ damage, is associated with future lower cognitive performance. Cardiac damage is also associated with lower cognitive performance independently of brain damage. Presence of damage in multiple organs might indicate a higher hypertensive disease burden, which might not all be visible on brain MRI (yet). These results could help to identify patients at high risk of developing cognitive problems. 


\section{REFERENCES}

1. Faraco G, ladecola C. Hypertension: A harbinger of stroke and dementia. Hypertension. 2013;62:810-817.

2. Veglio F, Paglieri C, Rabbia F, Bisbocci D, Bergui M, Cerrato P. Hypertension and cerebrovascular damage. Atherosclerosis. 2009;205:331-341.

3. De Groot JC, de Leeuw F-E, Oudkerk M, van Gijn J, Hofman A, Jolles J, Breteler MM. Cerebral white matter lesions and cognitive function: the Rotterdam Scan Study. Ann Neurol. 2000;47:145-151.

4. Pantoni L, Poggesi A, Inzitari D. The relation between white-matter lesions and cognition. Curr Opin Neurol. 2007;20:390-397.

5. Van der Flier WM, van Straaten EC, Barkhof F, Verdelho A, Madureira S, Pantoni L, Inzitari D, et al. Small vessel disease and general cognitive function in nondisabled elderly: The LADIS study. Stroke. 2005;36:2116-2120.

6. Carey CL, Kramer JH, Josephson SA, Mungas D, Reed BR, Schuff N, Weiner MW, et al. Subcortical lacunes are associated with executive dysfunction in cognitively normal elderly. Stroke. 2008;39:397-402.

7. Elias MF, Sullivan LM, Elias PK, D'Agostino RB, Wolf PA, Seshadri S, Au R, et al. Left ventricular mass, blood pressure, and lowered cognitive performance in the Framingham offspring. Hypertension. 2007;49:439-445.

8. Hayakawa M, Yano Y, Kuroki K, Inoue R, Nakanishi C, Sagara S, Koga M, et al. Independent association of cognitive dysfunction with cardiac hypertrophy irrespective of 24-h or sleep blood pressure in older hypertensives. Am J Hypertens. 2012;25:657.

9. Kähönen-Väre M, Brunni-Hakala S, Lindroos M, Pitkala K, Strandberg T, Tilvis R. Left ventricular hypertrophy and blood pressure as predictors of cognitive decline in old age. Aging Clin Exp Res. 2004;16:147-152.

10. Barzilay JI, Gao P, O'Donnell M, Mann JF, Anderson C, Fagard R, Probstfield J, et al. Albuminuria and decline in cognitive function: The ONTARGET/TRANSCEND studies. Arch Intern Med. 2011;171:142-150.

11. Deckers K, Camerino I, van Boxtel MP, Verhey FR, Irving K, Brayne C, Kivipelto M, et al. Dementia risk in renal dysfunction: A systematic review and meta-analysis of prospective studies. Neurology. 2017;88:198-208.

12. Henskens LH, van Oostenbrugge RJ, Kroon AA, Hofman PA, Lodder J, de Leeuw PW. Detection of silent cerebrovascular disease refines risk stratification of hypertensive patients. J Hypertens. 2009;27:846-853.

13. Van der Veen PH, Geerlings MI, Visseren FL, Nathoe HM, Mali WP, van der Graaf Y, Muller M. Hypertensive target organ damage and longitudinal changes in brain structure and function - The second manifestations of arterial disease-magnetic resonance study. Hypertension. 2015;66:1152-1158.

14. Henskens LH, van Oostenbrugge RJ, Kroon AA, de Leeuw PW, Lodder J. Brain microbleeds are associated with ambulatory blood pressure levels in a hypertensive population. Hypertension. 2008;51:62-68.

15. Huijts M, Duits A, van Oostenbrugge RJ, Kroon AA, de Leeuw PW, Staals J. Accumulation of MRI markers of cerebral small vessel disease is associated with decreased cognitive function: A study in first-ever lacunar stroke and hypertensive patients. Front Aging Neurosci. 2013;5:72.

16. Staals J, Makin SD, Doubal FN, Dennis MS, Wardlaw JM. Stroke subtype, vascular risk factors, and total MRI brain small-vessel disease burden. Neurology. 2014;83:1228-1234.

17. Fazekas F, Chawluk JB, Alavi A, Hurtig HI, Zimmerman RA. MR signal abnormalities at 1.5 T in Alzheimer's dementia and normal aging. AJR Am J Roentgenol. 1987;149:351-356.

18. Wardlaw JM, Smith EE, Biessels GJ, Cordonnier C, Fazekas F, Frayne R, Lindley RI, et al. Neuroimaging standards 


\section{8 | Chapter 5}

for research into small vessel disease and its contribution to ageing and neurodegeneration. Lancet Neurol. 2013;12:822-838.

19. Lang RM, Bierig M, Devereux RB, Flachskampf FA, Foster E, Pellikka PA, Picard MH, et al. Recommendations for chamber quantification. Eur Heart J Cardiovasc Imaging. 2006;7:79-108.

20. Uiterwijk R, Huijts M, Staals J, Duits A, Gronenschild E, Kroon AA, de Leeuw PW, et al. Subjective cognitive failures in patients with hypertension are related to cognitive performance and cerebral microbleeds. Hypertension. 2014;64:653-657.

21. Brand N, Jolles J. Learning and retrieval rate of words presented auditorily and visually. J Gen Psychol. 1985;112:201-210.

22. Wechsler D. WAIS III, nederlandstalige bewerking: technische handleiding. Lisse: Swets Test Publishers; 2001.

23. Golden CJ. Stroop Colour and Word Test. Chicago (IL): Stoelting; 1978.

24. Reitan R. Trail Making Test: Manual for administration, scoring and interpretation. Bloomington: Indiana University; 1956.

25. Luteyn F. Een nieuwe verkorte GIT. Dutch J Psychol. 1966;2:675-682.

26. Lezak MD, Howieson DB, Loring DW. Neuropsychological assessment. New York: Oxford University Press; 2004.

27. Schmand B, Bakker D, Saan R, Louman J. The Dutch Reading Test for Adults: a measure of premorbid intelligence level. Tijdschr Gerontol Geriatr. 1991;22:15-19. 




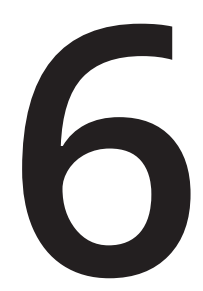

\section{Total cerebral small vessel disease MRI score is associated with cognitive decline in executive function in patients with hypertension}

Uiterwijk R, van Oostenbrugge RJ, Huijts M, de Leeuw PW, Kroon AA, Staals J 


\begin{abstract}
Objectives Hypertension is a major risk factor for white matter hyperintensities, lacunes, cerebral microbleeds and perivascular spaces, which are MRI markers of cerebral small vessel disease (SVD). Studies have shown associations between these individual MRI markers and cognitive functioning and decline. Recently, a "total SVD score" was proposed in which the different MRI markers were combined into one measure of SVD, to capture total SVD-related brain damage. We investigated if this SVD score was associated with cognitive decline over 4 years in patients with hypertension.
\end{abstract}

Methods In this Iongitudinal cohort study, 130 hypertensive patients (91 patients with uncomplicated hypertension and 39 hypertensive patients with a lacunar stroke) were included. They underwent a neuropsychological assessment at baseline and after 4 years. The presence of white matter hyperintensities, lacunes, cerebral microbleeds, and perivascular spaces were rated on baseline MRI. Presence of each individual marker was added to calculate the total SVD score (range 0-4) in each patient.

Results Uncorrected linear regression analyses showed associations between SVD score and decline in overall cognition ( $p=0.017)$, executive functioning $(p<0.001)$ and information processing speed ( $p=0.037$ ), but not with memory ( $p=0.911)$. The association between SVD score and decline in overall cognition and executive function remained significant after adjustment for age, sex, education, anxiety and depression score, potential vascular risk factors, patient group, and baseline cognitive performance.

Conclusion Our study shows that a total SVD score can predict cognitive decline, specifically in executive function, over 4 years in hypertensive patients. This emphasizes the importance of considering total brain damage due to SVD. 


\section{INTRODUCTION}

Hypertension is a major risk factor for brain damage such as white matter hyperintensities (WMH), lacunes, cerebral microbleeds and perivascular spaces. ${ }^{1}$ These MRI markers of brain damage result from small vessel disease (SVD). Several cross-sectional studies have investigated the implications of these MRI markers on cognition and showed significant associations with lower cognitive functioning or cognitive impairment. ${ }^{2.5} \mathrm{Also}$ in longitudinal research, associations between decline in cognitive functioning and $\mathrm{WMH}$ and lacunes have been found. ${ }^{6.9}$ Studies in cerebral microbleeds and perivascular spaces are more sparse, but a few studies have reported that these might also be related to cognitive decline or a higher risk of incident dementia. ${ }^{10,11}$

These previous studies mostly focused on the different MRI markers of SVD separately. There are some studies investigating the combined effect of WMH and lacunes on cognitive function or decline., 812 but no study combined all MRI markers. Recently, a "total SVD score" was proposed to combine all individual MRI markers into one measure of SVD, which aims to capture total brain damage from SVD. ${ }^{13}$ This total SVD score was found to be associated with higher blood pressure levels, ${ }^{14}$ hypertension, age, and other risk factors for SVD. ${ }^{13}$ Two recent cross-sectional studies have shown that this total SVD score was related to lower cognitive performance. ${ }^{15,16}$ However, the SVD score, combining all four markers, has never been applied in longitudinal research, studying cognitive decline.

The aim of the present study was to investigate if the SVD score was associated with cognitive decline over 4 years in hypertensive patients. To compose a patient cohort with a broad spectrum of severity of cerebral SVD, we included patients with uncomplicated hypertension as well as hypertensive patients with a prior clinical lacunar stroke. In addition, we examined if each of the MRI markers in this SVD score individually contributed to cognitive decline, in which it was expected that WMH make the largest contribution to cognitive decline.

\section{METHODS}

\section{Study population}

We included patients with hypertension, who were selected from two larger studies: the hypertension and brain damage study (HYBRiD) ${ }^{17}$ and the cognitive function in small vessel stroke study. ${ }^{15}$

In HYBRiD, essential hypertensive patients were recruited from the hypertension outpatient clinic of the Department of Internal Medicine of Maastricht University Medical Centre, the 
Netherlands. Hypertension was defined as an off-medication, clinically measured conventional blood pressure $\geq 140 \mathrm{~mm}$ Hg systolic and/or $\geq 90 \mathrm{~mm} \mathrm{Hg}$ diastolic. Exclusion criteria were a history of symptomatic cardio- or cerebrovascular disease or contraindications for MRI. Details about the HYBRiD study have been described before. ${ }^{17}$ A total of 218 patients were included in the HYBRiD study and they were asked to participate in the present study.

In the cognitive function in small vessel stroke study, first-ever lacunar stroke patients were recruited from the Neurology Department of the Maastricht University Medical Centre between February 2009 and July 2012. Lacunar stroke was defined as an acute stroke syndrome with a small $(<20 \mathrm{~mm})$ ischemic lesion on acute brain MR in the brain stem, basal ganglia or internal capsule, compatible with the occlusion of a single perforating small artery. If no such lesion was visible, we used established clinical criteria for lacunar stroke. ${ }^{18}$ Patients with severe comorbidity, either neurological or psychiatric, were excluded. Furthermore, patients with possible other causes for the lacunar stroke than cerebral SVD (cardiac embolic source, cerebral large vessel disease, or carotid stenosis), were also excluded. ${ }^{15} \mathrm{~A}$ total of 77 patients were included in the small vessel stroke study, and those who had hypertension (defined as the use of antihypertensive medication or a history of hypertension) were selected for the present analysis.

All patients underwent a brain MRI scan and a neuropsychological assessment. For hypertensive lacunar stroke patients, the neuropsychological assessment was performed 3 months after stroke to exclude acute phase effects. The neuropsychological assessment was repeated 4 years later. Registration of educational level was based on the Dutch classification system "Verhage" (in which level 1, 2, 3 and 4 are considered as low education, level 5 is considered as middle education and level 6 and 7 are considered as high education. ${ }^{19}$ Information about vascular risk factors (body mass index (BMI), smoking, and the presence of diabetes mellitus or hypercholesterolemia) was obtained based on patient self-report.

The Medical Ethics Committee of the Maastricht University Medical Centre approved this study and all participants gave written informed consent.

\section{MRI data}

All HYBRiD patients were scanned on a 1.5T MRI scanner, while the lacunar stroke patients were scanned using a 1.5T or a 3T scanner (both scanners: Philips Medical Systems, Best, The Netherlands). For lacunar stroke patients, the median time between stroke and MRI was 6 days (IQR=3.5 to 32.5). On brain MRI scans (standard axial T2-weighted, FLAIR and $\mathrm{T}^{*}$ gradient echo sequences, details are described in supplementary material) two experienced vascular neurologists individually rated the markers of SVD. The inter-rater agreement statistics have been previously reported. ${ }^{15}$ An ordinal scale representing the 
total burden of SVD was created. Definitions and rating method have been described in detail before. ${ }^{13}$ In short, the presence of each of the four MRI markers for SVD (WMH, lacunes, cerebral microbleeds and perivascular spaces) was counted to retrieve a total SVD score (ranging 0-4). WMH were assessed according to the Fazekas scale. ${ }^{20}$ Presence of WMH was defined as periventricular WMH Fazekas score 3 (irregular hyperintensities extending into the deep white matter) and/or deep WMH Fazekas score 2 or 3 (confluent hyperintensities). Lacunes and cerebral microbleeds were assessed according to the international consensus definition ${ }^{21}$ and for both lacunes and cerebral microbleeds, one point was awarded in the total SVD score if at least one lacune or cerebral microbleed was present. The recent symptomatic small subcortical infarct in the lacunar stroke patients was not counted as a lacune, because the SVD score intends to rate background SVD burden. Perivascular spaces were rated as mild, moderate or extensive at the level of the basal ganglia, since perivascular spaces in the basal ganglia are specifically related to SVD. ${ }^{22,23}$ One point was awarded for moderate or extensive perivascular spaces in the basal ganglia.

\section{Neuropsychological assessment}

Cognitive performance was measured with a comprehensive neuropsychological assessment at baseline and after 4 years of follow-up, as has been described before. ${ }^{24}$ The test protocol was identical in both patient groups. Memory domain was measured with the Rey Auditory Verbal Learning Test ${ }^{25}$ (immediate recall, delayed recall and delayed recognition) and the Digit Span Forward (subtest of Wechsler Adult Intelligence Scale (WAIS)-III26). Executive function domain was measured with the Stroop Colour Word Test ${ }^{27}$ (SCWT) interference score (time of part 3 minus mean time of parts 1 and 2), Trail Making Test (TMT) ${ }^{28}$ interference score (time of part B minus time of part A), Category (animals and professions ${ }^{29}$ and Letter Fluency, ${ }^{30}$ Letter-Number Sequencing (subtest of WAIS-III), and Digit Span Backward (subtest of WAIS-III). Information processing speed domain was measured with the Symbol Substitution - Coding (subtest of WAIS-III), TMT part A, and SCWT parts 1 and 2. Parallel versions were used for baseline and follow up assessment for the Rey Auditory Verbal Learning Test and the Letter Fluency test.

For each patient, we subtracted the raw test scores at follow-up from test scores at baseline. These raw test decline scores were transformed into standardized values (z-scores), by dividing the difference between the individual raw score and the overall group sample mean by the overall group sample standard deviation (SD). Z-scores of all tests within one domain were averaged to receive cognitive decline compound scores of each domain. In addition, an overall cognitive decline compound score was calculated by averaging the three domain compound scores. Higher compound scores indicated higher decline. Z-scores of tests with higher scores representing worse performance (e.g. SCWT and TMT) were inverted before computing the compound scores. 
The Rotterdam - Cambridge Cognitive Examination (R-CAMCOG) was used to determine the presence of possible dementia, defined as a score $<34 .{ }^{31}$ Symptoms of depression and anxiety were measured using the Hospital Anxiety and Depression Scale (HADS) and the total score (range 0-42) was used. Because symptoms possibly caused by physical problems (e.g. insomnia or weight loss) are not included in the HADS, the scale is considered to be suitable to use in somatic populations. ${ }^{32}$

\section{Statistical analysis}

Differences in baseline characteristics between uncomplicated hypertensive patients and hypertensive lacunar stroke patients were investigated using independent t-test, chisquare test or Mann-Whitney $U$ test. The associations between SVD score and age, sex, HADS total score, low or high educational level, and vascular risk factors were examined using spearman's correlation analysis or Mann-Whitney $U$ test. Associations between the SVD score and cognitive decline (overall cognition and separate domains of executive functioning, information processing speed and memory) were investigated using simple linear regression analyses. Next, the association between SVD score and cognitive decline was adjusted for possible confounders in three models of multivariable linear regression analyses. In model 1, the associations between SVD score and cognitive decline scores were adjusted for age, sex, educational level, HADS total score and potential confounding vascular risk factors (BMI, smoking, and the presence of diabetes mellitus or hypercholesterolemia). To avoid overfitting, we only included those vascular risk factors that were associated with cognitive decline with $p<0.10$ in a simple regression model. In model 2 , we corrected for the occurrence of a symptomatic stroke, by adding patient group (patient with uncomplicated hypertension or hypertensive lacunar stroke patient) to the previous model. In model 3, baseline cognitive performance (of the corresponding cognitive domain) was added to model 2.

Coefficients of determinations $\left(R^{2}\right)$ were calculated in simple linear regression analyses to determine the proportion of variance in cognitive decline explained by the SVD score. To investigate the contribution of each of the markers separately, we repeated the analyses with $\mathrm{WMH}$, lacunes, microbleeds and perivascular spaces as predictor individually (dichotomised, as they were defined in the SVD score) and $R^{2 \prime}$ s were calculated. In addition, $R^{2}$ of the full WMH Fazekas score (deep and periventricular scores summed, score ranging 0-6) was calculated.

IBM SPSS Statistics 22 software was used for all analyses. Results were considered significant at $p<0.05$. 
Figure 1. Participant flow diagram

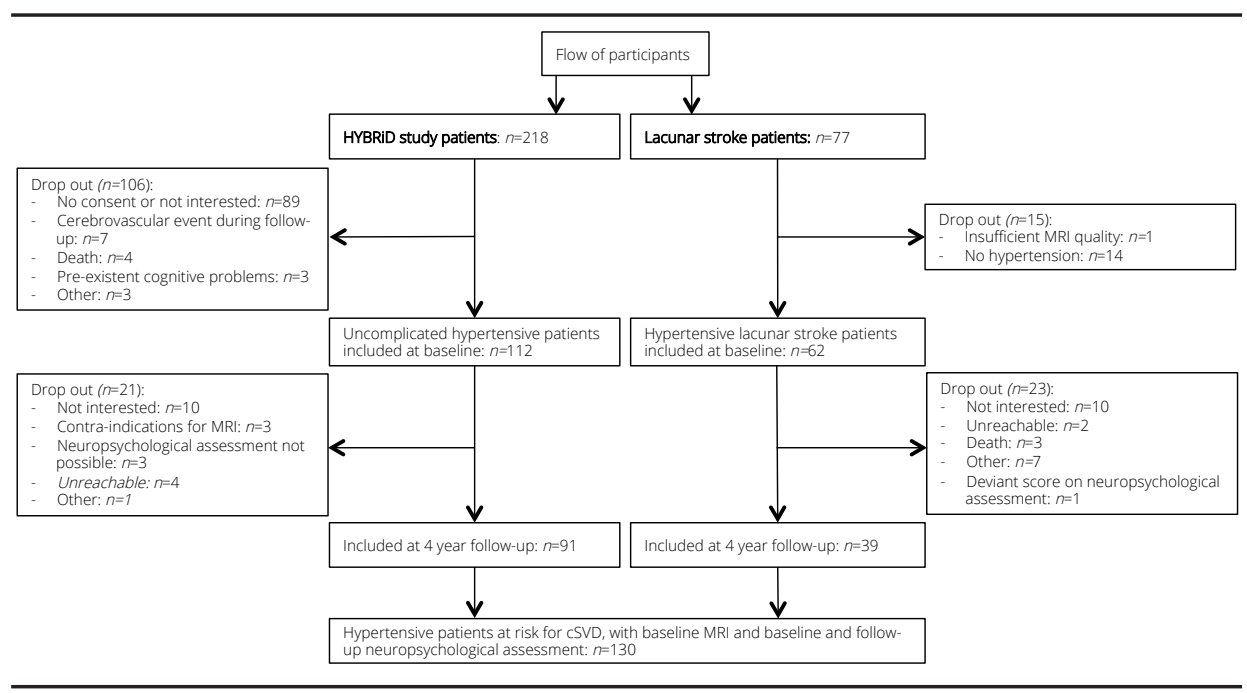

\section{RESULTS}

\section{Participants}

Flow of participants is shown in figure 1. We included 112 HYBRiD hypertensive patients and 62 hypertensive lacunar stroke patients in the present study at baseline. Of these, 91 hypertensive patients and 39 lacunar stroke patients completed 4-year follow-up, giving 130 patients in this study. The mean follow-up period was 4.04 year $(S D=0.10)$. Included patients $(n=130)$ did not differ from the excluded patients ( $n=165)$ in age $(58.7 \pm 12.2$ versus $59.3 \pm 13.5$ years, respectively, $p=0.70$ ) or sex (male $57.7 \%$ versus $47.0 \%$, respectively, $p=0.09$ ). Patients who completed follow-up ( $n=130)$ did not differ from patients who only completed baseline ( $n=44$ ) regarding sex (male $57.7 \%$ versus $59.1 \%$, respectively, $p=0.87$ ), but were younger (58.7 \pm 12.1 versus 65.1 \pm 13.9 , respectively, $p=0.004$ ) and had lower baseline overall cognition compound scores ( $p=0.012)$. Baseline characteristics, including educational level, HADS score and vascular risk factors, for the overall group and separately for uncomplicated hypertensive patients and lacunar stroke patients are shown in table 1. Hypertensive lacunar stroke patients were older ( $p=0.001)$, more often had hypercholesterolemia $(p<0.001)$, more often smoked $(p=0.02)$, had lower educational levels ( $p=0.03$ ), and had higher SVD scores compared to uncomplicated hypertensive patients $(p<0.001)$. Other baseline characteristics did not differ between uncomplicated hypertensive patients and lacunar stroke patients. Hypertensive lacunar stroke patients had a lower overall cognition score at baseline compared to uncomplicated hypertensive 
patients $(p<0.001)$, but there were no differences in cognitive decline between patient groups ( $p=0.707$ ). Three lacunar stroke patients had a recurrent stroke during follow-up, while none of the uncomplicated hypertensive patients had a stroke during follow-up.

Table 1. Patients' characteristics for uncomplicated hypertensive patients and hypertensive lacunar stroke patients

\begin{tabular}{llll}
\hline Baseline characteristic & All patients $(n=130)$ & $\begin{array}{l}\text { Uncomplicated } \\
\text { hypertensive } \\
\text { patients }(n=91)\end{array}$ & $\begin{array}{l}\text { Hypertensive lacunar } \\
\text { stroke patients } \\
(n=39)\end{array}$ \\
\hline Age, mean (SD), years & $58.7(12.2)$ & $56.3(11.9)$ & $64.2(11.1)$ \\
Male sex, No. (\%) & $75(57.7 \%)$ & $52(57.1 \%)$ & $23(59.0 \%)$ \\
Educational level, No. (\%): & & & \\
- Low & $42(32.3 \%)$ & $23(25.3 \%)$ & $19(48.7 \%)$ \\
- Average & $42(32.3 \%)$ & $33(36.3 \%)$ & $9(23.1 \%)$ \\
- High & $46(35.4 \%)$ & $35(38.5 \%)$ & $11(28.2 \%)$ \\
HADS total score, median (IQR) & $8(4-13)$ & $7(4-12)$ & $9(4-14)$ \\
Diabetes Mellitus, No. (\%) & $3(2.3 \%)$ & $1(1.1 \%)$ & $2(5.1 \%)$ \\
Hypercholesterolemia, No. (\%)* & $57(44.9 \%)$ & $31(34.8 \%)$ & $26(68.4 \%)$ \\
Current smoking, No. (\%) & $27(20.8 \%)$ & $14(15.4 \%)$ & $13(33.3 \%)$ \\
Body Mass Index, mean (SD), kg/m² & $27.3(4.2)$ & $27.4(4.2)$ & $27.0(4.0)$ \\
SVD score 0, No. (\%) & $63(48.5 \%)$ & $50(54.9 \%)$ & $13(33.3 \%)$ \\
SVD score 1, No. (\%) & $33(25.4 \%)$ & $27(29.7 \%)$ & $6(15.4 \%)$ \\
SVD score 2, No. (\%) & $18(13.8 \%)$ & $8(8.8 \%)$ & $10(25.6 \%)$ \\
SVD score 3, No. (\%) & $13(10.0 \%)$ & $6(6.6 \%)$ & $7(17.9 \%)$ \\
SVD score 4, No. (\%) & $3(2.3 \%)$ & $0(0.0 \%)$ & $3(7.7 \%)$ \\
\hline
\end{tabular}

$S D=$ standard deviation; HADS=hospital anxiety and depression scale; SVD=small vessel disease

* Information about hypercholesterolemia was missing for 3 patients ( 2 uncomplicated hypertensive patients and 1 hypertensive lacunar stroke patient)

For two patients, one baseline neuropsychological test score in the domain of executive function was missing. Therefore, for these patients the executive function decline score was composed of the remaining 5 tests in this domain. For three patients, the baseline Rey Auditory Verbal Learning Test was missing. Since this test determines 3 out of 4 test scores of the memory domain, no reliable memory decline score could be formed for these two patients and consequently the overall cognition decline score was also missing. As a result, analyses of executive function and information processing speed decline are based on the data of 130 patients, while analyses of overall cognition and memory decline are based on the data of 127 patients. 


\section{SVD score}

Presence of each category of the SVD score is shown in table 1. The SVD score correlated with age $(p<0.001)$ and lower BMI $(p=0.043)$, but there were no significant differences in sex, educational level, HADS total score, or vascular risk factors.

\section{SVD score and cognitive decline}

At baseline three patients (2\%; 1 uncomplicated hypertensive patient and 2 lacunar stroke patients) had an R-CAMCOG score <34, indicating possible dementia. At follow-up, besides these three patients, six (5\%) other patients had a score $<34$. Three of these patients were uncomplicated hypertensive patients and 6 were hypertensive patients with a lacunar stroke $(p=0.021)$.

Unadjusted analyses showed an association between SVD score and cognitive decline in overall cognition, executive functioning and information processing speed, but not with memory (table 2). The association between SVD score and decline in executive function remained significant after adjustment for age, sex, educational level, HADS score and potential vascular risk factors (table 2, model 1). Additional adjustment for patient group with or without additional adjustment for baseline cognition (of the corresponding cognitive domain) showed significant associations between SVD score and decline in overall cognition and executive function (table 2, models 2 and 3).

Correlations of determination $\left(R^{2} \mathrm{~S}\right)$, indicating the proportions of variance in cognitive decline explained by SVD score or each of the individual MRI markers, are shown in table 3. The magnitude of the contributions of the individual markers (as defined in the SVD score) differed across domains, as could be seen from the $R^{21} s$ of the individual markers in table 3. The dichotomized WMH explained a smaller proportion of variance in all cognitive domains, except memory, than the SVD score. The proportions of variance in the three cognitive domains explained by the WMH total Fazekas score were comparable to those explained by the SVD score; in overall cognitive decline the WMH total Fazekas score explained a higher proportion of variance than the SVD score. 
Table 2. Association between small vessel disease score and cognitive decline

\begin{tabular}{|c|c|c|c|c|}
\hline & $\begin{array}{l}\text { Decline in overall } \\
\text { cognition; } \\
B(95 \% \mathrm{Cl})\end{array}$ & $p$ & $\begin{array}{l}\text { Decline in executive } \\
\text { function; } \\
B(95 \% \mathrm{Cl})\end{array}$ & $p$ \\
\hline Simple regression analysis & $0.08(0.02$ to 0.15$)$ & 0.017 & 0.17 (0.09 to 0.24$)$ & $<0.001$ \\
\hline Multivariable regression analysis model 1 & $0.07(-0.01$ to 0.14$)$ & 0.072 & $0.13 *(0.05$ to 0.21$)$ & 0.003 \\
\hline Multivariable regression analysis model 2 & 0.08 (0.006 to 0.16) & 0.036 & $0.13 *(0.04$ to 0.21$)$ & 0.005 \\
\hline Multivariable regression analysis model 3 & $0.09(0.02$ to 0.16$)$ & 0.012 & $0.13^{*}(0.05$ to 0.22$)$ & 0.003 \\
\hline \multicolumn{5}{|l|}{ Table 2 continued } \\
\hline & $\begin{array}{l}\text { Decline in } \\
\text { information } \\
\text { processing speed; } \\
B(95 \% \mathrm{Cl})\end{array}$ & $p$ & $\begin{array}{l}\text { Decline in memory; } \\
B(95 \% \mathrm{Cl})\end{array}$ & $p$ \\
\hline Simple regression analysis & 0.11 (0.007 to 0.22) & 0.037 & $-0.006(-0.11$ to 0.10$)$ & 0.911 \\
\hline Multivariable regression analysis model 1 & $0.08(-0.04$ to 0.20$)$ & 0.179 & $0.001(-0.11$ to 0.12$)$ & 0.984 \\
\hline Multivariable regression analysis model 2 & $0.10(-0.03$ to 0.22$)$ & 0.118 & $0.04(-0.08$ to 0.15$)$ & 0.548 \\
\hline Multivariable regression analysis model 3 & $0.11(-0.004$ to 0.23$)$ & 0.059 & $0.05(-0.04$ to 0.15$)$ & 0.271 \\
\hline
\end{tabular}

$B=$ unstandardized regression coefficient; $\mathrm{Cl}=$ confidence interval

Model 1: correction for age, sex, educational level, HADS score and potential vascular risk factors (those that were associated with cognitive decline with $p<0.10$ in a simple regression model)*

Model 2: correction for age, sex, educational level, HADS score, potential vascular risk factors and patient group Model 3: correction for age, sex, educational level, HADS score, potential vascular risk factors, patient group and baseline cognition

* Model includes baseline body mass index

Table 3. $R^{2}$ for the association between cognitive decline and small vessel disease score versus individual MRI markers

\begin{tabular}{lllll}
\hline & $\begin{array}{l}\text { Overall } \\
\text { cognition; } \\
\boldsymbol{R}^{2}(\boldsymbol{p})\end{array}$ & $\begin{array}{l}\text { Executive } \\
\text { function; } \\
\boldsymbol{R}^{2}(\boldsymbol{p})\end{array}$ & $\begin{array}{l}\text { Information } \\
\text { processing } \\
\text { speed; } \boldsymbol{R}^{2}(\boldsymbol{p})\end{array}$ & $\begin{array}{l}\text { Memory; } \\
\boldsymbol{R}^{2}(\boldsymbol{p})\end{array}$ \\
\hline SVD score & $4.5 \%(0.017)$ & $13.0 \%(<0.001)$ & $3.4 \%(0.037)$ & $0.0 \%(0.911)$ \\
WMH total Fazekas score & $7.8 \%(0.001)$ & $12.9 \%(<0.001)$ & $4.3 \%(0.017)$ & $1.0 \%(0.259)$ \\
WMH (dichotomized)* & $1.7 \%(0.139)$ & $6.5 \%(0.003)$ & $1.6 \%(0.150)$ & $0.0 \%(0.938)$ \\
Presence of lacunes* & $2.1 \%(0.100)$ & $9.6 \%(<0.001)$ & $0.0 \%(0.879)$ & $0.2 \%(0.590)$ \\
Presence of microbleeds* & $1.4 \%(0.180)$ & $1.2 \%(0.222)$ & $3.9 \%(0.024)$ & $0.0 \%(0.816)$ \\
Moderate-severe perivascular spaces* & $2.5 \%(0.077)$ & $7.6 \%(0.002)$ & $3.7 \%(0.028)$ & $0.4 \%(0.489)$ \\
\hline
\end{tabular}

$R^{2}=$ coefficient of determination; SVD=small vessel disease; $\mathrm{WMH}=$ white matter hyperintensities

*Presence of individual markers were defined as in the SVD score 


\section{DISCUSSION}

We showed that a total SVD score, that captures total SVD-related brain damage on MRI imaging, was associated with cognitive decline in executive function over 4 years in patients with hypertension.

Previous studies showed associations between cognitive decline and baseline degree of WMH $^{6,8}$ or number of lacunes. ${ }^{6,33}$ However, these studies have investigated associations with individual markers of cerebral SVD and no other longitudinal study on cognitive decline considered the total extent of SVD. By capturing all different MRI markers of cerebral SVD in one measure, we think that the SVD score better represents the underlying severity of the disease than just one MRI marker.

Some of the MRI markers of cerebral SVD might be considered more important than others in the association with cognitive function. Our results showed that while the dichotomized WMH explained smaller proportions of variance in cognitive decline compared to the SVD score, the WMH total Fazekas score explained similar or even higher proportions of variance in cognitive decline. This might be explained by a wider range of severity that is captured by the Fazekas scale (score ranging 0-6) compared to the SVD score (ranging 0-4). We believe that the SVD score is an alternative to WMH Fazekas scale and provides a more complete overview of all SVD-related brain damage. We advocate that all of these four markers should be considered together when studying the consequences of SVD-related brain damage.

Besides the four MRI markers of cerebral SVD included in the SVD score, other markers of brain damage might influence cognitive decline, such as cerebral atrophy ${ }^{34}$ or hippocampal volume..$^{35}$ Whether cerebral atrophy is part of SVD is still a point of discussion. Cerebral atrophy is not specific for SVD, since it also occurs in normal aging or can be related to focal injury. Atrophy was found to be only marginally associated with SVD score. ${ }^{13}$ However, the unavailability of an atrophy measure in our data should be considered a limitation of our study. In addition, all MRI markers in the SVD score are dichotomized, so degrees of WMH and perivascular spaces or number of lacunes or microbleeds were not accounted for in the score. Future research might test different points in the SVD score for different degrees and thereby may lead to a more optimal SVD score in predicting cognitive decline. Finally, the SVD score includes perivascular spaces in the basal ganglia, but not in the white matter. This can however be justified because PVS in the basal ganglia, not those in the white matter, are associated with SVD in terms of lacunar stroke, 22,23 white matter hyperintensities, ${ }^{22}$ progression of $\mathrm{WMH}_{1}{ }^{36}$ blood pressure ${ }^{37}$ and cognition. ${ }^{38}$ PVS in the white matter seem to be related to amyloid angiopathy. ${ }^{39}$ 
The SVD score has been studied in association with cognitive performance before, but these studies were cross-sectional. We published before ${ }^{15}$ on a cohort of 189 lacunar stroke and hypertension patients, of which our present study cohort is a subset consisting of those patients who had hypertension and follow-up measurements. In our cross-sectional baseline study, SVD score was related to information processing speed and overall cognition, after correction for age and sex. Another cross-sectional study ${ }^{16}$ in an age-cohort of 680 older participants showed that the SVD score is associated with general cognitive ability. Additional latent variable modelling analyses was also performed in that study, which showed that the $4 \mathrm{MRI}$ markers are indeed jointly indicative of an underlying overall SVD state.

The finding that only decline in executive function is associated with the SVD score might indicate an expression of subcortical damage caused by SVD. The result is in line with a recent study ${ }^{40}$ in patients with lacunar stroke and leukoaraiosis, in which patients showed a significant decline in executive function, but not in other domains (processing speed, working memory and global cognition).

A major strength is the longitudinal design of our study with a four-year follow-up period. In addition, all patients received a comprehensive neuropsychological assessment, which included multiple tests in 3 cognitive domains. A limitation of the study is the use of variable MRI field strength. Therefore, we performed sub-analyses including only the patients who were scanned at 1.5T ( $n=109)$. This showed not only significant associations between the SVD score and cognitive decline in overall cognition and executive functioning, but also with information processing speed, when we used the same variables as in multivariable regression model 3 (results not shown). The second limitation is the mixed hypertensive population. To reach a relatively large sample with a broad spectrum of severity of cerebral SVD, we included both hypertensive patients with and without a small vessel stroke. Stroke itself might have influenced cognition. However, the inclusion of patient group as a confounder in the model did not change the results. In addition, three lacunar stroke patients had a recurrent stroke during follow-up, which might have influenced cognitive decline. However, results did not change when we excluded these three patients from analyses (results not shown). Still, we studied a relatively healthy population; a large part of the patient group had a SVD score of 0 . This might reduce the generalizability of the results to more affected patient populations. In addition, results may differ in other SVD populations, such as in SVD patients with intracerebral hemorrhage. Lastly, due to selection bias cognitively healthier people might be included in the study at baseline, which might have led to an underestimation of the reported associations. Future studies are needed to investigate the association between the SVD score and cognitive decline in a more affected and more homogeneous population. 
This longitudinal study investigated a combined score of small vessel disease MRI markers in relation to cognitive decline and shows that this score predicts future cognitive decline in executive functioning. The SVD score might be a good alternative to the Fazekas WMH score in predicting cognitive decline, since the SVD score might provide a more complete overview of total SVD-related brain damage. 


\section{REFERENCES}

1. Veglio F, Paglieri C, Rabbia F, Bisbocci D, Bergui M, Cerrato P. Hypertension and cerebrovascular damage. Atherosclerosis. 2009;205:331-341.

2. De GrootJC, de Leeuw F-E, Oudkerk M, van Gijn J, Hofman A, Jolles J, Breteler MM. Cerebral white matter lesions and cognitive function: the Rotterdam Scan Study. Ann Neurol. 2000;47:145-151.

3. Van der Flier WM, van Straaten EC, Barkhof F, Verdelho A, Madureira S, Pantoni L, Inzitari D, et al. Small vessel disease and general cognitive function in nondisabled elderly: The LADIS study. Stroke. 2005;36:2116-2120.

4. Pantoni L, Poggesi A, Inzitari D. The relation between white-matter lesions and cognition. Curr Opin Neurol. 2007;20:390-397.

5. Carey CL, Kramer JH, Josephson SA, Mungas D, Reed BR, Schuff N, Weiner MW, et al. Subcortical lacunes are associated with executive dysfunction in cognitively normal elderly. Stroke. 2008;39:397-402.

6. Prins ND, van Dijk EJ, den Heijer T, Vermeer SE, Jolles J, Koudstaal PJ, Hofman A, et al. Cerebral small-vessel disease and decline in information processing speed, executive function and memory. Brain. 2005;128:20342041.

7. Pavlovic AM, Pekmezovic T, Tomic G, Trajkovic JZ, Sternic N. Baseline predictors of cognitive decline in patients with cerebral small vessel disease. J Alzheimers Dis. 2014;42:S37-S43.

8. Jokinen H, Gouw A, Madureira S, Ylikoski R, van Straaten E, van Der Flier W, Barkhof F, et al. Incident lacunes influence cognitive decline: The LADIS study. Neurology. 2011;76:1872-1878.

9. Vermeer SE, Prins ND, den Heijer T, Hofman A, Koudstaal PJ, Breteler MM. Silent brain infarcts and the risk of dementia and cognitive decline. N Eng/J Med. 2003;348:1215-1222.

10. Gregoire S, Smith K, Jäger H, Benjamin M, Kallis C, Brown M, Cipolotti L, et al. Cerebral microbleeds and longterm cognitive outcome: Longitudinal cohort study of stroke clinic patients. Cerebrovasc Dis. 2012;33:430-435.

11. Zhu Y-C, Dufouil C, Soumaré A, Mazoyer B, Chabriat H, Tzourio C. High degree of dilated Virchow-Robin spaces on MRI is associated with increased risk of dementia. J Alzheimers Dis. 2010;22:663-672.

12. Baune BT, Roesler A, Knecht S, Berger K. Single and combined effects of cerebral white matter lesions and lacunar infarctions on cognitive function in an elderly population. J Gerontol A Bio/ Sci Med Sci. 2009;64A:118124.

13. Staals J, Makin SD, Doubal FN, Dennis MS, Wardlaw JM. Stroke subtype, vascular risk factors, and total MRI brain small-vessel disease burden. Neurology. 2014;83:1228-1234.

14. Klarenbeek P, van Oostenbrugge RJ, Rouhl RP, Knottnerus IL, Staals J. Ambulatory blood pressure in patients with lacunar stroke: Association with total MRI burden of cerebral small vessel disease. Stroke. 2013;44:29952999.

15. Huijts M, Duits A, van Oostenbrugge RJ, Kroon AA, de Leeuw PW, Staals J. Accumulation of MRI markers of cerebral small vessel disease is associated with decreased cognitive function: A study in first-ever lacunar stroke and hypertensive patients. Front Aging Neurosci. 2013;5:72

16. Staals J, Booth T, Morris Z, Bastin ME, Gow AJ, Corley J, Redmond P, et al. Total MRI load of cerebral small vessel disease and cognitive ability in older people. Neurobiol Aging. 2015;36:2806-2811.

17. Henskens LH, van Oostenbrugge RJ, Kroon AA, de Leeuw PW, Lodder J. Brain microbleeds are associated with 
ambulatory blood pressure levels in a hypertensive population. Hypertension. 2008;51:62-68.

18. De Jong G, Kessels F, Lodder J. Two types of lacunar infarcts further arguments from a study on prognosis. Stroke. 2002;33:2072-2076.

19. Verhage F. Intelligentie en leeftijd: onderzoek bij Nederlanders van twaalf tot zevenenzeventig jaar. Assen: Van Gorcum 1964.

20. Fazekas F, Chawluk JB, Alavi A, Hurtig HI, Zimmerman RA. MR signal abnormalities at 1.5 T in Alzheimer's dementia and normal aging. AJR Am J Roentgenol. 1987;149:351-356.

21. Wardlaw JM, Smith EE, Biessels GJ, Cordonnier C, Fazekas F, Frayne R, Lindley RI, et al. Neuroimaging standards for research into small vessel disease and its contribution to ageing and neurodegeneration. Lancet Neurol. 2013;12:822-838.

22. Doubal FN, MacLullich AM, Ferguson KJ, Dennis MS, Wardlaw JM. Enlarged perivascular spaces on MRI are a feature of cerebral small vessel disease. Stroke. 2010;41:450-454.

23. Potter GM, Doubal FN, Jackson CA, Chappell FM, Sudlow CL, Dennis MS, Wardlaw JM. Enlarged perivascular spaces and cerebral small vessel disease. Int J Stroke. 2015;10:376-381.

24. Uiterwijk R, Huijts M, Staals J, Duits A, Gronenschild E, Kroon AA, de Leeuw PW, et al. Subjective cognitive failures in patients with hypertension are related to cognitive performance and cerebral microbleeds. Hypertension. 2014;64:653-657.

25. Brand N, Jolles J. Learning and retrieval rate of words presented auditorily and visually. J Gen Psychol. $1985 ; 112: 201-210$.

26. Wechsler D. WAIS III, nederlandstalige bewerking: technische handleiding. Lisse: Swets Test Publishers; 2001.

27. Golden CJ. Stroop Colour and Word Test. Chicago (IL): Stoelting; 1978.

28. Reitan R. Trail Making Test: Manual for administration, scoring and interpretation. Bloomington: Indiana University; 1956.

29. Luteyn F. Een nieuwe verkorte GIT. Dutch J Psychol. 1966;2:675-682.

30. Lezak MD, Howieson DB, Loring DW. Neuropsychological assessment. New York: Oxford University Press; 2004.

31. De Koning I, van Kooten F, Koudstaal PJ, Dippel DW. Diagnostic value of the Rotterdam-CAMCOG in post-stroke dementia. J Neurol Neurosurg Psychiatry. 2005;76:263-265.

32. Spinhoven PH, Ormel J, Sloekers PP, Kempen GIJ, Speckens AE, van Hemert AM. A validation study of the Hospital Anxiety and Depression Scale (HADS) in different groups of Dutch subjects. Psychol Med. 1997;27:363-370.

33. Imamine R, Kawamura T, Umemura T, Umegaki H, Kawano N, Hotta M, Kouchi Y, et al. Does cerebral small vessel disease predict future decline of cognitive function in elderly people with type 2 diabetes? Diabetes Res Clin Pract. 2011;94:91-99.

34. Lawrence AJ, Patel B, Morris RG, MacKinnon AD, Rich PM, Barrick TR, Markus HS. Mechanisms of cognitive impairment in cerebral small vessel disease: multimodal MRI results from the St George's cognition and neuroimaging in stroke (SCANS) study. PloS one. 2013;8:e61014.

35. O'Brien JT, Lloyd A, McKeith I, Gholkar A, Ferrier N. A longitudinal study of hippocampal volume, cortisol levels, and cognition in older depressed subjects. American Journal of Psychiatry. 2004;161:2081-2090.

36. Loos CM, Klarenbeek P, van Oostenbrugge RJ, Staals J. Association between perivascular spaces and progression of white matter hyperintensities in lacunar stroke patients. PloS one. 2015;10:e0137323. 


\section{6 | Chapter 6}

37. Klarenbeek P, van Oostenbrugge RJ, Lodder J, Rouhl RP, Knottnerus IL, Staals J. Higher ambulatory blood pressure relates to enlarged Virchow-Robin spaces in first-ever lacunar stroke patients.J Neurol. 2013;260:115-121.

38. Huijts M, Duits A, Staals J, Kroon AA, de Leeuw PW, van Oostenbrugge RJ. Basal ganglia enlarged perivascular spaces are linked to cognitive function in patients with cerebral small vessel disease. Curr Neurovasc Res. 2014;11:136-141.

39. Charidimou A, Hong YT, Jäger HR, Fox Z, Aigbirhio Fl, Fryer TD, Menon DK, et al. White matter perivascular spaces on magnetic resonance imaging: Marker of cerebrovascular amyloid burden? Stroke. 2015;46:17071709.

40. Lawrence AJ, Brookes RL, Zeestraten EA, Barrick TR, Morris RG, Markus HS. Pattern and rate of cognitive decline in cerebral small vessel disease: A prospective study. PloS one. 2015;10:e0135523. 


\section{SUPPLEMENTARY MATERIAL}

\section{Supplementary MRI data}

MRI sequence parameters for the uncomplicated hypertensive patients (all patients were scanned on a 1.5T MRI scanner: Philips Medical Systems, Best, The Netherlands):

T2-weighted turbo spin echo sequence (TR/TE=4820/100 ms), T2*-weighted gradient echo sequence (TR/TE=736/23 ms), and FLAIR sequence (TR/TI/TE=8000/2000/120), all with thickness of $5 \mathrm{~mm}$ and $0.5 \mathrm{~mm}$ interslice gap.

MRI sequence parameters for the hypertensive lacunar stroke patients: (18 patients were scanned on a 1.5T and 21 patients on a 3T MRI scanner, both scanners: Philips Medical Systems, Best, The Netherlands):

1.5T: T2-weighted turbo spin echo sequence (TR/TE=shortest(5515)/100 ms), T2* weighted gradient echo sequence (TR/TE=shortest(851)/23 ms) and FLAIR sequence (TR/TI/TE=8000/2000/120 ms), all with slice thickness $5 \mathrm{~mm}$ and $0.5 \mathrm{~mm}$ interslice gap.

3.0T: T2-weighted turbo spin echo sequence (TR/TE=3000/80 ms), T2*-weighted gradient echo sequence (TR/TE=shortest(794)/16 ms) and FLAIR sequence (TR/TI/ $\mathrm{TE}=11000 / 2800 / 125 \mathrm{~ms}$ ), all with slice thickness $5 \mathrm{~mm}$ and $0.5 \mathrm{~mm}$ interslice gap. 



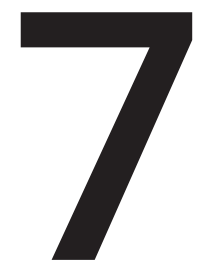

\section{MRI progression of cerebral small vessel disease and cognitive decline in patients with hypertension}

Uiterwijk R, Staals J, Huijts M, de Leeuw PW, Kroon AA, van Oostenbrugge RJ

Published in Journal of Hypertension, 2017; 35: 1263-1270 


\begin{abstract}
Objectives Hypertension is associated with cognitive deficits, probably because it is a major risk factor for the development of white matter hyperintensities (WMH), lacunes, and cerebral microbleeds, which are MRI markers of cerebral small vessel disease (CSVD). Studies into associations between presence or progression of these MRI markers and cognitive decline in hypertensive patients are rare. We investigated the association of baseline presence and progression of MRI markers of CSVD with cognitive decline over 4 years in patients with hypertension.
\end{abstract}

Methods In this longitudinal study, hypertensive patients underwent neuropsychological assessments and brain MRI at baseline and after 4 years. Presence and progression of periventricular and subcortical WMH, lacunes and cerebral microbleeds were visually rated.

Results 128 hypertensive patients (90 patients with essential hypertension and 38 hypertensive lacunar stroke patients), mean age: $58.6 \pm 12.2$ years, were included. Progression of periventricular WMH was associated with cognitive decline in simple regression analysis ( $p=0.001)$ and in multivariable analysis with correction for baseline WMH presence and potential confounders ( $p=0.004)$. In this multivariable analysis, $R^{2}$ of progression of periventricular $\mathrm{WMH}$ was 5.6\%, while $R^{2}$ of baseline presence of periventricular $\mathrm{WMH}$ was $0.6 \%$. We did not find significant associations between baseline presence or progression of the other MRI markers and cognitive decline.

Conclusion In patients with hypertension, progression of periventricular WMH over 4 years is associated with cognitive decline, while we could not show an association between baseline periventricular WMH and cognitive decline. These results emphasize the importance of preventing progression of $\mathrm{WMH}$ in hypertensive patients. 


\section{INTRODUCTION}

Hypertension is a risk factor for cognitive deficits and vascular dementia, probably because hypertension induces microvascular dysfunction in the brain. ${ }^{1}$ This leads to brain damage, such as white matter hyperintensities (WMH), lacunes and cerebral microbleeds. These are MRI markers of cerebral Small Vessel Disease (CSVD).

Cross-sectional studies have shown that the MRI markers of CSVD are associated with lower cognitive functioning. ${ }^{2-6}$ The implications of these MRI markers on future cognitive decline have been investigated less extensively, but a number of studies have shown that the presence of MRI markers predicts cognitive decline.-9 Only a few studies have investigated the increase or progression of MRI markers in relation to cognitive decline longitudinally. Associations between WMH progression and cognitive decline have been shown. ${ }^{10-13}$ However, most of the studies did not differentiate between periventricular and subcortical/deep WMH, despite the fact that cross-sectional data showed that location of WMH might influence the impact on cognition.,214,15 In addition, only very few studies have shown associations between new lacunes ${ }^{10,16,17}$ or microbleeds ${ }^{18}$ and cognitive decline. Finally, it is unclear whether the progression of SVD is more predictive of cognitive decline than the presence of SVD at baseline.

Most of these earlier studies were population studies. The association between progression of SVD and cognitive decline has not been assessed before specifically in hypertensive patients, even though it is known that high blood pressure is associated with an increased risk for developing SVD ${ }^{19}$ and with progression of MRI markers. ${ }^{20}$ More insight into predictors of cognitive decline in this population at risk is needed. Therefore, the aim of the present study was to investigate the association of both baseline presence and progression of MRI markers of CSVD with cognitive decline over 4 years in patients with hypertension.

\section{METHODS}

\section{Study population}

To have a wide range of severity of CSVD on MRI, we included both uncomplicated hypertensive patients, and hypertensive patients who had suffered a lacunar stroke due to CSVD. Patients were selected from two larger studies: uncomplicated hypertensive patients from the Hypertension and Brain Damage study (HYBRiD) $)^{21}$ and hypertensive lacunar stroke patients from the Cognitive function in small vessel stroke study. ${ }^{22}$ 
In the HYBRiD study, essential hypertensive patients were recruited from the hypertension outpatient clinic of the Department of Internal Medicine of Maastricht University Medical Centre, the Netherlands. Hypertension was defined as an off-medication, office measured conventional blood pressure $\geq 140 \mathrm{~mm} \mathrm{Hg}$ systolic and/or $\geq 90 \mathrm{~mm} \mathrm{Hg}$ diastolic. Exclusion criteria were a history of symptomatic cardio- or cerebrovascular disease or contraindications for MRI. Details about the HYBRiD study have been described before..$^{21} \mathrm{~A}$ total of 218 patients were included in the HYBRiD study and they were asked to participate in the present follow-up study.

In the Cognitive function in small vessel stroke study, first-ever lacunar stroke patients were recruited from the Neurology Department of the Maastricht University Medical Centre between February 2009 and July 2012. Lacunar stroke was defined as an acute stroke syndrome with a small $(<20 \mathrm{~mm})$ non-cortical ischemic lesion on acute brain MR, compatible with the occlusion of a single perforating small artery. If no such lesion was visible, we used established clinical criteria for lacunar stroke. ${ }^{23}$ Patients with severe comorbidity, either neurological or psychiatric, were excluded. Furthermore, patients with possible other causes for the lacunar stroke than cerebral SVD (cardiac embolic source, cerebral large vessel disease, or carotid stenosis), were also excluded. ${ }^{22}$ A total of 77 patients were included in the small vessel stroke study, and those who had hypertension (defined as the use of antihypertensive medication or a history of hypertension) were selected for the present study and asked for follow-up.

All patients underwent a brain MRI scan and a neuropsychological assessment, which were repeated after 4 years. For hypertensive lacunar stroke patients, the baseline neuropsychological assessment was performed 3 months after stroke to exclude acute phase effects. Registration of educational level was based on the Dutch classification system "Verhage" (in which level 1, 2, 3 and 4 are considered as low education, level 5 is considered as middle education and level 6 and 7 are considered as high education). ${ }^{24}$ Information about vascular risk factors (body mass index (BMI), smoking, and the presence of diabetes mellitus or hypercholesterolemia [defined as the use of lipid-lowering drugs and/or a known total cholesterol level $>5.0 \mathrm{mmol} / \mathrm{l}$ for lacunar stroke patients or $>6.5 \mathrm{mmol} / \mathrm{l}$ for uncomplicated hypertensive patients]) was obtained. At follow-up, three automatic, oscillometric office blood pressure measurements were taken in sitting position, after 10 minutes of rest and with 5 minutes between each measurement. Mean systolic and diastolic blood pressure was calculated by averaging the second and third measurements.

The Medical Ethics Committee of the Maastricht University Medical Centre approved this study and all participants gave written informed consent. 


\section{MRI data}

All uncomplicated hypertensive patients were scanned twice on a 1.5T MRI scanner, while the lacunar stroke patients were scanned twice using a 1.5T or a 3T scanner (both scanners: Philips Medical Systems, Best, The Netherlands). Patients were scanned at the same scanner at baseline and at follow-up. On brain MRI scans (standard axial T2-weighted, FLAIR and T2* gradient echo sequences) two experienced vascular neurologists rated baseline MRI markers and one rated progression of markers, after reaching satisfactory inter-rater agreements. The inter-rater agreement statistics have been previously reported. ${ }^{22}$ Baseline WMH were assessed according to the Fazekas scale. ${ }^{25}$ Extensive periventricular WMH was defined as periventricular WMH Fazekas score 3 (irregular hyperintensities extending into the deep matter) and extensive subcortical/deep WMH was defined as deep WMH Fazekas score 2 or 3 (confluent hyperintensities). Lacunes and cerebral microbleeds were counted according to international consensus definition. ${ }^{26}$ In lacunar stroke patients, the symptomatic lacunar infarct was not counted as lacune.

Progression of periventricular and subcortical WMH were scored according to the WMH change scale as proposed by Prins et al. ${ }^{27}$ Progression of periventricular WMH was scored in three regions (frontal caps, lateral band and occipital caps) and progression of subcortical WMH is scored in four regions (frontal, parietal, temporal and occipital). New lesions or increase of existing lesions was scored in each region as +1 and disappeared lesions or decrease of existing lesions was scored as -1. This adds up to a progression score ranging from -3 to +3 for periventricular WMH and -4 to +4 for subcortical WMH. We defined progression of periventricular or subcortical $\mathrm{WMH}$ as a score of $\geq 1$. Progression of lacunes or microbleeds was defined as any new lacune or microbleed.

\section{Neuropsychological assessment}

Cognitive performance was measured with a comprehensive neuropsychological assessment at baseline and after 4 years of follow-up, as has been described before. ${ }^{28}$ Both patient groups received identical test protocols. Executive function domain was measured with the Stroop Colour Word Test ${ }^{29}$ (SCWT) interference score (time of part 3 minus mean time of parts 1 and 2), Trail Making Test ${ }^{30}$ (TMT) interference score (time of part B minus time of part A), Category (animals and professions) ${ }^{31}$ and Letter ${ }^{32}$ Verbal Fluency, Letter-Number Sequencing (subtest of Wechsler Adult Intelligence Scale (WAIS)-III) ${ }^{33}$, and Digit Span Backward (subtest of WAIS-III). Information processing speed domain was measured with the Symbol Substitution - Coding (subtest of WAIS-III), TMT part A, and SCWT parts 1 and 2. Memory domain was measured with the Rey Auditory Verbal Learning Test ${ }^{34}$ (immediate recall, delayed recall and delayed recognition) and the Digit Span Forward (subtest of WAIS-III). Parallel versions were used for baseline and follow up assessment for the Rey Auditory Verbal Learning Test and the Letter Fluency test. 
For each patient, raw test scores at follow-up were subtracted from test scores at baseline. Standardized values (z-scores) were created by dividing the difference between the individual raw score and the overall group sample mean by the overall group sample standard deviation (SD). Z-scores of all tests within one domain were averaged to obtain cognitive decline compound scores of each domain. Next, the overall cognitive decline compound score was calculated by averaging the three domain compound scores. Higher compound scores indicated more decline. Z-scores of tests with higher scores representing worse performance (e.g. SCWT and TMT) were inverted before computing the compound scores.

To determine the presence of possible dementia, the Rotterdam - Cambridge Cognitive Examination (R-CAMCOG) was used, in which a cutoff score of $<34$ was applied. ${ }^{35}$ The Hospital Anxiety and Depression Scale (HADS) total score (range 0-42) was used to determine symptoms of depression and anxiety. Since symptoms possibly caused by physical problems (e.g. insomnia or weight loss) are not included in the HADS, the scale is considered to be suitable to use in somatic populations. ${ }^{36}$

\section{Statistical analysis}

Associations between baseline MRI markers and progression of MRI markers of SVD, and cognitive decline in overall cognition were investigated using simple linear regression analyses (model 1). Next, the associations of baseline MRI markers and progression of MRI markers with cognitive decline, independent of each other, were examined in a multivariable regression analyses (model 2). Model 2 was repeated for the two patient groups (uncomplicated hypertensive patient or hypertensive lacunar stroke patient) separately to examine if associations were similar in each group. Lastly, the associations were corrected for age, sex, educational level, HADS total score, patient group, baseline cognitive performance and potential confounding vascular risk factors (BMI, smoking, the presence of diabetes mellitus or hypercholesterolemia, and systolic and diastolic blood pressure) (model 3). To avoid overfitting, we only included those vascular risk factors that were associated with cognitive decline with $p<0.10$ in a simple regression model. In all models, besides the unstandardized regression coefficient, 95\% confidence interval and significance level, the $R^{2}$ was calculated to show the proportion of variance in cognitive decline explained by either the baseline MRI marker or the progression of MRI marker. The full multivariable regression model including all covariates (model 3) was repeated for the cognitive domains of executive functioning, information processing speed and memory. IBM SPSS Statistics 22 software was used for all analyses. Results were considered significant at $p<0.05$. 
Figure 1. Participant flow diagram

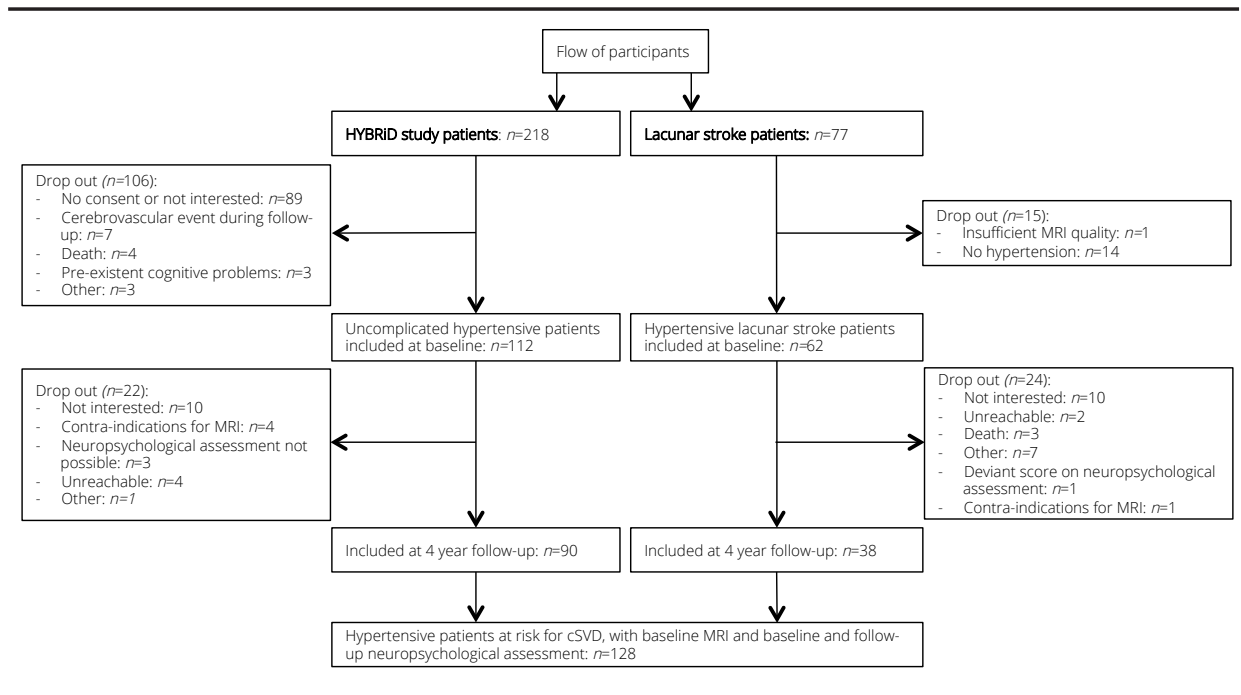

\section{RESULTS}

\section{Participants}

Flow of participants is shown in figure 1. We included 112 uncomplicated hypertensive patients and 62 hypertensive lacunar stroke patients in the present study at baseline. Of these, 90 uncomplicated hypertensive patients and 38 lacunar stroke patients completed neuropsychological assessment and brain MRI at 4-year follow-up, which adds up to 128 patients in this study. The mean follow-up period was 4.04 year $(S D=0.10)$. Included patients ( $n=128$ ) did not differ from the excluded patients $(n=167)$ in age or sex. Patients who completed follow-up ( $n=128$ ) did not differ from patients who only completed baseline $(n=46)$ regarding sex (male $58.6 \%$ versus 56.5\%, respectively, $p=0.81$ ), but they were younger (58.6 \pm 12.2 versus $65.1 \pm 13.5$, respectively, $p=0.003$ ) and had better overall cognition at baseline $(p=0.014)$. Baseline characteristics for the overall group and separately for uncomplicated hypertensive patients and lacunar stroke patients are shown in table 1. Decline in overall cognition was not different between these two patient groups ( $p=0.720$ ).

The number of patients with baseline presence and progression of each MRI marker of SVD are shown in table 2. Three lacunar stroke patients had a recurrent stroke during follow-up, while none of the uncomplicated hypertensive patients had a stroke during follow-up. 
Table 1. Patients' characteristics for uncomplicated hypertensive patients and hypertensive lacunar stroke patients

\begin{tabular}{llll}
\hline Baseline characteristic & $\begin{array}{l}\text { All patients } \\
(\boldsymbol{n = 1 2 8 )}\end{array}$ & $\begin{array}{l}\text { Uncomplicated } \\
\text { hypertensive } \\
\text { patients }(\boldsymbol{n}=90)\end{array}$ & $\begin{array}{l}\text { Hypertensive } \\
\text { lacunar stroke } \\
\text { patients }(\boldsymbol{n}=38)\end{array}$ \\
\hline Age, mean (SD), years & $58.6(12.2)$ & $56.2(11.9)$ & $64.1(11.2)$ \\
Male sex, No. (\%) & $75(58.6)$ & $52(57.8)$ & $23(60.5)$ \\
Educational level, No. (\%) & & & $18(47.4)$ \\
- Low & $40(31.3)$ & $22(24.4)$ & $9(23.7)$ \\
- Average & $42(32.8)$ & $33(36.7)$ & $11(28.9)$ \\
- High & $46(35.9)$ & $35(38.9)$ & $8.5(4-14)$ \\
HADS total score, median (IQR) & $8(4-13)$ & $7(4-12)$ & $1(2.6)$ \\
Diabetes Mellitus, No. (\%) & $2(1.6)$ & $1(1.1)$ & $25(67.6)$ \\
Hypercholesterolemia, No. (\%)* & $55(44.0)$ & $30(34.1)$ & $12(31.6)$ \\
Current smoking, No. (\%) & $26(20.3)$ & $14(15.6)$ & $27.0(4.1)$ \\
BMl, mean (SD), kg/m² & $27.3(4.2)$ & $27.4(4.2)$ & $145.7(21.4)$ \\
SBP, mean (SD), mmHG & $145.5(17.9)$ & $145.4(16.4)$ & $82.4(14.3)$ \\
DBP, mean (SD), mmHG & $84.2(11.7)$ & $84.9(10.4)$ & \\
\hline
\end{tabular}

$S D=$ standard deviation; HADS=hospital anxiety and depression scale; IQR=interquartile range; BMI=body mass index $\mathrm{SBP}=$ systolic blood pressure; $\mathrm{DBP}=$ diastolic blood pressure

* Information about hypercholesterolemia was missing for 3 patients ( 2 uncomplicated hypertensive patients and

1 hypertensive lacunar stroke patient)

Table 2. Baseline presence and progression of MRI markers of cSVD

\begin{tabular}{llll}
\hline MRI marker & $\begin{array}{l}\text { All patients } \\
(n=128)\end{array}$ & $\begin{array}{l}\text { Uncomplicated } \\
\text { hypertensive } \\
\text { patients }(\boldsymbol{n}=90)\end{array}$ & $\begin{array}{l}\text { Hypertensive } \\
\text { lacunar stroke } \\
\text { patients }(\boldsymbol{n}=38)\end{array}$ \\
\hline Baseline presence of cSVD & & & $7(18.4)$ \\
Extensive periventricular WMH, No. (\%) & $17(13.3)$ & $10(11.1)$ & $7(18.4)$ \\
Extensive deep WMH, No. (\%) & $13(10.2)$ & $6(6.7)$ & $10(26.3)$ \\
Microbleeds, No. (\%) & $25(19.5)$ & $15(16.7)$ & $19(50.0)$ \\
Asymptomatic lacunes, No. (\%) & $40(31.3)$ & $21(23.3)$ & $15(40.5)$ \\
Progression of cSVD & & & $22(59.5)$ \\
Progression of periventricular WMH, No. (\%)* & $37(29.4)$ & $22(24.7)$ & $9(24.3)$ \\
Progression of subcortical WMH, No. (\%)* & $56(44.4)$ & $34(38.2)$ & $9(23.7)$ \\
Incident microbleeds, No. (\%)* & $14(11.0)$ & $5(5.6)$ &
\end{tabular}

CSVD=cerebral small vessel disease; $\mathrm{WMH}=$ white matter hyperintensities

* Information about progression of periventricular and subcortical WMH was missing for 2 patients (1 in each patient subgroup) and information about incident microbleeds was missing for one lacunar stroke patient 
For two patients, one baseline neuropsychological test score in the domain of executive function was missing. Therefore, for these patients the executive function decline score was composed of the remaining 5 tests in this domain. For three patients, the baseline Rey Auditory Verbal Learning Test was missing. Since this test determines 3 out of 4 test scores of the memory domain, no reliable memory decline score could be formed for these two patients and consequently the overall cognition decline score was also missing. Brain MRI of two patients was of insufficient quality to determine progression of $\mathrm{WMH}$. In addition, in one of these patients incident microbleeds could not be determined, due to insufficient quality of the T2* gradient echo image. As a result of these missing neuropsychological tests and missing MRI data, the number of patients in each analysis slightly differed.

At baseline three patients had an R-CAMCOG score <34, indicating possible dementia. At follow-up, besides these three patients, six other patients had a score $<34$.

\section{MRI markers and cognitive decline}

In simple linear regression analyses, baseline periventricular WMH presence was not associated with cognitive decline, but the association between periventricular WMH progression and cognitive decline was significant (model 1; table 3).

In multivariable regression analyses, periventricular WMH progression remained significantly associated with cognitive decline, independent of baseline WMH presence (model 2; table 3) and also additionally independent of age, sex, educational level, HADS total score, patient group and baseline cognition (model 3; table 3). There were no vascular risk factors included in the final model because there were no associations with $p<0.10$ between any of the vascular risk factors and overall cognitive decline in simple linear regression analyses. In this final multivariable model 3, baseline periventricular WMH only explained $0.6 \%$ of variance in cognitive decline, while progression of periventricular WMH explained 5.6\%.

For both baseline and progression of subcortical WMH, no significant associations were found in any of the statistical models. Although non-significant, proportions of explained variance of cognitive decline were higher for baseline subcortical WMH presence than for WMH progression (table 3). For baseline as well as progression of microbleeds or lacunes, none of the associations were significant and $R^{2 \prime} s$ were comparable for baseline presence and progression of the marker (table 4).

When performing model 2 for both patient groups separately, results were similar to the results in the total group. The association between progression of periventricular WMH and cognitive decline was significant for both uncomplicated hypertensive patients and lacunar stroke patients $(B(95 \% \mathrm{Cl})=0.24(0.03$ to 0.44$), p=0.026$, and $B(95 \% \mathrm{Cl})=0.36(0.08$ to 
Table 3. Associations between WMH and overall cognitive decline

\begin{tabular}{|c|c|c|c|c|}
\hline & \multicolumn{3}{|c|}{ Periventricular WMH } & \multirow[b]{2}{*}{$R^{2}$ change } \\
\hline & $B$ & $95 \% \mathrm{Cl}$ & $p$ & \\
\hline 1a. Simple regression analysis: baseline $\mathrm{WMH}$ & 0.15 & -0.07 to 0.38 & 0.185 & $1.4 \%$ \\
\hline 1b. Simple regression analysis: progression WMH & 0.27 & 0.11 to 0.43 & 0.001 & $8.5 \%$ \\
\hline 2. Multivariable analysis & & & & $\dagger$ \\
\hline Baseline WMH & 0.08 & -0.15 to 0.30 & 0.491 & $0.4 \%$ \\
\hline Progression WMH & 0.26 & 0.10 to 0.42 & 0.002 & $7.4 \%$ \\
\hline 3. Multivariable analysis including covariates* & & & & $\ddagger$ \\
\hline Baseline WMH & 0.10 & -0.12 to 0.32 & 0.364 & $0.6 \%$ \\
\hline Progression WMH & 0.24 & 0.08 to 0.40 & 0.004 & $5.6 \%$ \\
\hline \multicolumn{5}{|l|}{ Table 3 continued } \\
\hline & \multicolumn{2}{|c|}{ Subcortical WMH } & & \\
\hline & $B$ & $95 \% \mathrm{Cl}$ & $p$ & $R^{2}$ change \\
\hline 1a. Simple regression analysis: baseline $\mathrm{WMH}$ & 0.23 & -0.02 to 0.48 & 0.066 & $2.7 \%$ \\
\hline 1b. Simple regression analysis: progression WMH & 0.06 & -0.09 to 0.21 & 0.426 & $0.5 \%$ \\
\hline 2. Multivariable analysis & & & & $\S$ \\
\hline Baseline WMH & 0.22 & -0.04 to 0.48 & 0.097 & $2.3 \%$ \\
\hline Progression WMH & 0.03 & -0.13 to 0.18 & 0.735 & $0.1 \%$ \\
\hline 3. Multivariable analysis including covariates* & & & & $\|$ \\
\hline Baseline WMH & 0.26 & -0.001 to 0.52 & 0.051 & $2.7 \%$ \\
\hline Progression WMH & -0.009 & -0.16 to 0.14 & 0.910 & $0.0 \%$ \\
\hline
\end{tabular}

WMH=white matter hyperintensities; $B=$ unstandardized regression coefficient; $C l=$ confidence interval; $R^{2}=$ coefficient of determination

* Including age, sex, educational level, HADS total score, patient group, baseline cognition

† Total $R^{2}$ of the model $=8.9 \%$

$\ddagger$ Total $R^{2}$ of the model $=25.1 \%$

$\S$ Total $R^{2}$ of the model $=2.8 \%$

$\|$ Total $R^{2}$ of the model $=21.0 \%$

0.65), $p=0.015$, respectively), while the associations between baseline periventricular WMH and cognitive decline were not significant in both patient groups. Associations between cognitive decline and the other MRI markers (subcortical WMH, microbleeds and lacunes), both baseline and progression, were all non-significant in both patient groups.

\section{MRI markers and decline in separate cognitive domains}

In simple linear regression analyses between each of the vascular risk factors and cognitive decline, associations with $\mathrm{p}<0.10$ were found between $\mathrm{BMI}$ and decline in executive function and between smoking and decline in information processing speed. These factors were included in the respective multivariable regression analyses.

Multivariable regression analyses, with correction for age, sex, educational level, HADS total score, patient group, baseline cognition of the corresponding cognitive domain, and 
Table 4. Associations between microbleeds and lacunes and overall cognitive decline

\begin{tabular}{|c|c|c|c|c|}
\hline & \multicolumn{4}{|c|}{ Microbleeds } \\
\hline & $B$ & $95 \% \mathrm{Cl}$ & $p$ & $R^{2}$ change \\
\hline 1a. Simple regression analysis: baseline marker & 0.13 & -0.05 to 0.32 & 0.160 & $1.6 \%$ \\
\hline 1b. Simple regression analysis: progression marker & 0.15 & -0.10 to 0.41 & 0.229 & $1.2 \%$ \\
\hline 2. Multivariable analysis & & & & $\dagger$ \\
\hline Baseline marker & 0.12 & -0.08 to 0.31 & 0.251 & $1.1 \%$ \\
\hline Progression marker & 0.11 & -0.15 to 0.37 & 0.390 & $0.6 \%$ \\
\hline 3. Multivariable analysis including covariates* & & & & $\ddagger$ \\
\hline Baseline marker & 0.14 & -0.06 to 0.33 & 0.168 & $1.3 \%$ \\
\hline Progression marker & 0.08 & -0.18 to 0.34 & 0.541 & $0.3 \%$ \\
\hline \multicolumn{5}{|l|}{ Table 4 continued } \\
\hline & \multicolumn{4}{|c|}{ Lacunes } \\
\hline & $B$ & $95 \% \mathrm{Cl}$ & $p$ & $R^{2}$ change \\
\hline 1a. Simple regression analysis: baseline marker & 0.13 & -0.03 to 0.29 & 0.109 & $2.1 \%$ \\
\hline 1b. Simple regression analysis: progression marker & 0.18 & -0.05 to 0.41 & 0.126 & $1.9 \%$ \\
\hline 2. Multivariable analysis & & & & $\S$ \\
\hline Baseline marker & 0.12 & -0.04 to 0.28 & 0.139 & $1.8 \%$ \\
\hline Progression marker & 0.17 & -0.07 to 0.40 & 0.161 & $1.6 \%$ \\
\hline 3. Multivariable analysis including covariates* & & & & $\|$ \\
\hline Baseline marker & 0.10 & -0.06 to 0.26 & 0.222 & $1.0 \%$ \\
\hline Progression marker & 0.17 & -0.06 to 0.40 & 0.152 & $1.4 \%$ \\
\hline
\end{tabular}

$B=$ unstandardized regression coefficient; $C$ I=confidence interval; $R^{2}=$ coefficient of determination

* Including age, sex, educational level, HADS total score, patient group, baseline cognition

$\dagger$ Total $R^{2}$ of the model $=2.3 \%$

$\neq$ Total $R^{2}$ of the model $=20.1 \%$

$\S$ Total $R^{2}$ of the model $=3.6 \%$

$\|$ Total $R^{2}$ of the model $=20.4 \%$

vascular risk factors (as explained above), showed significant associations between executive functioning and progression of periventricular WMH, baseline presence of subcortical $\mathrm{WMH}$, and baseline presence of lacunes. No significant associations were found between information processing speed or memory and any of the MRI markers. For tables with results, please see supplementary material.

\section{DISCUSSION}

Our results show that progression of periventricular WMH over 4 years is associated with cognitive decline, especially in the domain of executive functioning, in patients with hypertension. Furthermore, progression is more important in predicting cognitive decline than periventricular WMH already present at baseline. We could not show associations between 
progression of subcortical WMH, microbleeds or lacunes and overall cognitive decline.

Other studies also showed that progression of periventricular WMH is associated with cognitive decline. Van den Heuvel et al. ${ }^{11}$ showed periventricular WMH progression to be related with decline in executive functioning in a non-demented elderly population and Van Dijk et al. ${ }^{10}$ showed it to be related with decline in general cognitive function and information processing speed in a population-based cohort study. In agreement with our study, both studies also found no associations between progression of subcortical WMH and cognitive decline. Most other studies that showed progression of $\mathrm{WMH}$ to be related with cognitive decline did not differentiate between periventricular and subcortical $\mathrm{WMH} .{ }^{12,13}$ In a cross-sectional study, De Groot et al. ${ }^{2}$ found that the degree of periventricular WMH was associated with cognitive impairment, while the degree of subcortical WMH was not. They suggested that subcortical WMH probably disrupt short cortico-cortical connections, while periventricular WMH are more likely to disrupt long associating tracts that connect more distant cortical areas. Since the performance on neuropsychological tests is more likely to depend on the connection between many cortical areas, this could explain the reported difference between periventricular and subcortical WMH in associations with cognition.

We showed that progression of periventricular WMH explained 5.6\% of variance in cognitive decline, while baseline periventricular WMH only explained $0.6 \%$ of variance. All other covariates in the model, including age, together explained $18.3 \%$. Therefore, we think that preventing progression of periventricular WMH can make a relevant difference in the prevention of cognitive decline. However, most of the variance in cognitive decline is unexplained, so there are still many more factors predicting cognitive decline.

We could not find significant associations between occurrence of new microbleeds or lacunes and cognitive decline. A few other studies have shown associations between incident lacunes and cognitive decline. ${ }^{10,16,17}$ These studies not only had a larger sample size, but also a higher percentage of patients with incident lacunes, ranging from 12\% to $18.6 \%$. In addition, these studies were performed in other populations. For example, one study took the presence of WMH as an inclusion criterion. Since this is a predictor of future progression of SVD, this may explain the different results. ${ }^{16}$ Regarding incident microbleeds, we could only find one other study which addressed its association with cognitive decline. ${ }^{18}$ This study examined patients with cerebral autosomal dominant arteriopathy with subcortical infarcts and leukoencephalopathy (CADASIL) and showed that an increase in number of microbleeds over 7 years was associated with cognitive decline. However, the number of microbleeds (mean number 3.5, range 0-40) in these patients is much higher than in our population, which might explain the different results. Also, whether results found in patients with a genetic variant of CSVD, known for its progressive 
nature and strong association with cognitive decline, can be generalized to populations with common types of cSVD is questionable.

Studies have shown that active blood pressure lowering might stop or delay the progression of WMH in patients with and without cerebrovascular disease. ${ }^{37,38}$ In addition, a previous study has shown that blood pressure is most strongly associated with periventricular WMH compared to subcortical WMH. ${ }^{39}$ Since our results show that progression of periventricular WMH is associated with cognitive decline, it suggests the importance of good blood pressure control in patients with hypertension to prevent its progression. In our study, we did not have specific information about blood pressure control during the four years of follow-up, so we could not examine its effect on progression of WMH. This is an important limitation of our study.

Another limitation of the study is the use of variable MRI field strength. 107 of 128 patients (83.6\%) were scanned at a 1.5T MRI scanner and 21 (16.4\%) patients, all lacunar stroke patients, at a 3T MRI. In all patients, the same scanner was used for baseline and follow-up. However, rating of progression in patients scanned at 3T might be overestimated compared to $1.5 \mathrm{~T}$. We repeated the multivariable regression analyses in only those patients scanned at 1.5T. The association between progression of periventricular $\mathrm{WMH}$ and cognitive decline did not change. The second limitation is the mixed hypertensive population. To reach a relatively large sample with a substantial progression of cerebral SVD, we included both hypertensive patients with and without small vessel stroke. However, by including patient group as a confounder we corrected for the presence of a symptomatic stroke in medical history. In addition, we showed that results were similar in separate analyses for each patient group. Three lacunar stroke patients had a recurrent stroke during follow-up, which might have influenced cognitive decline. However, results did not change when we excluded these three patients from analyses (results not shown). Third, a visual scale was used to assess WMH presence and progression. The use of volumetric techniques might have been more sensitive. This could have led to an underestimation of the $R^{2}$ of presence or progression of $\mathrm{WMH}$ in the association with cognitive decline. In addition, an underestimation of cognitive decline might be caused by the higher drop-out rate of older and cognitively worse patients. Despite these limitations, a major strength is the longitudinal design of our study with a four-year follow-up period. In addition, all patients received extensive neuropsychological assessments, which included multiple tests in 3 cognitive domains.

In conclusion, we found that progression of periventricular WMH over 4 years is associated with cognitive decline in hypertensive patients, while we could not show an association between baseline periventricular WMH and cognitive decline. Therefore, these results 
show the importance of preventing progression of $\mathrm{WMH}$ in patients with hypertension. Since studies have shown that active blood pressure lowering might stop or delay the progression of WMH, future studies are needed to investigate if optimal blood pressure control that minimizes progression of $\mathrm{WMH}$ in patients with hypertension, can also prevent cognitive decline. In our study, we could not show significant associations between progression of other MRI markers of SVD and overall cognitive decline over 4 years. Since the proportion of patients with hypertension who developed new lacunes or microbleeds over time was relatively small, larger studies with a long follow-up period are needed to further investigate this. 


\section{REFERENCES}

1. Iadecola C. Hypertension and dementia. Hypertension. 2014;64:3-5.

2. De Groot JC, de Leeuw F-E, Oudkerk M, van Gijn J, Hofman A, Jolles J, Breteler MM. Cerebral white matter lesions and cognitive function: the Rotterdam Scan Study. Ann Neurol. 2000;47:145-151.

3. Van der Flier WM, van Straaten EC, Barkhof F, Verdelho A, Madureira S, Pantoni L, Inzitari D, et al. Small vessel disease and general cognitive function in nondisabled elderly: The LADIS study. Stroke. 2005;36:2116-2120.

4. Carey CL, Kramer JH, Josephson SA, Mungas D, Reed BR, Schuff N, Weiner MW, et al. Subcortical lacunes are associated with executive dysfunction in cognitively normal elderly. Stroke. 2008;39:397-402.

5. Van Norden AGW, van den Berg HAC, de Laat KF, Gons RAR, van Dijk EJ, de Leeuw F-E. Frontal and temporal microbleeds are related to cognitive function: The Radboud University Nijmegen Diffusion tensor and Magnetic resonance Cohort (RUN DMC) study. Stroke. 2011;42:3382-3386.

6. Werring DJ, Frazer DW, Coward LJ, Losseff NA, Watt H, Cipolotti L, Brown MM, et al. Cognitive dysfunction in patients with cerebral microbleeds on T2*-weighted gradient-echo MRI. Brain. 2004;127:2265-2275.

7. Prins ND, van Dijk EJ, den Heijer T, Vermeer SE, Jolles J, Koudstaal PJ, Hofman A, et al. Cerebral small-vessel disease and decline in information processing speed, executive function and memory. Brain. 2005;128:20342041.

8. Pavlovic AM, Pekmezovic T, Tomic G, Trajkovic JZ, Sternic N. Baseline predictors of cognitive decline in patients with cerebral small vessel disease. J Alzheimers Dis. 2014;42:S37-S43.

9. Jokinen H, Kalska H, Ylikoski R, Madureira S, Verdelho A, van der Flier WM, Scheltens P, et al. Longitudinal cognitive decline in subcortical ischemic vascular disease-the LADIS Study. Cerebrovasc Dis. 2009;27:384-391.

10. Van Dijk EJ, Prins ND, Vrooman HA, Hofman A, Koudstaal PJ, Breteler MM. Progression of cerebral small vessel disease in relation to risk factors and cognitive consequences: Rotterdam Scan Study. Stroke. 2008;39:27122719.

11. Van den Heuvel D, ten Dam V, de Craen A, Admiraal-Behloul F, Olofsen H, Bollen E, Jolles J, et al. Increase in periventricular white matter hyperintensities parallels decline in mental processing speed in a non-demented elderly population. J Neurol Neurosurg Psychiatry. 2006;77:149-153.

12. Longstreth W, Arnold AM, Beauchamp NJ, Manolio TA, Lefkowitz D, Jungreis C, Hirsch CH, et al. Incidence, manifestations, and predictors of worsening white matter on serial cranial magnetic resonance imaging in the elderly: The Cardiovascular Health Study. Stroke. 2005;36:56-61.

13. Schmidt R, Ropele S, Enzinger C, Petrovic K, Smith S, Schmidt H, Matthews PM, et al. White matter lesion progression, brain atrophy, and cognitive decline: The Austrian Stroke Prevention Study. Ann Neurol. 2005;58:610-616.

14. Yoon B, Shim YS, Cheong H-K, Hong Y-J, Lee K-S, Park KH, Ahn KJ, et al. White matter hyperintensities in mild cognitive impairment: clinical impact of location and interaction with lacunes and medial temporal atrophy. J Stroke Cerebrovasc Dis. 2014;23:e365-e372.

15. Soriano-Raya J, Miralbell J, López-Cancio E, Bargalló N, Arenillas JF, Barrios M, Cáceres C, et al. Deep versus periventricular white matter lesions and cognitive function in a community sample of middle-aged participants. J Int Neuropsychol Soc. 2012;18:874-885. 
16. Jokinen H, Gouw A, Madureira S, Ylikoski R, van Straaten E, van Der Flier W, Barkhof F, et al. Incident lacunes influence cognitive decline: The LADIS study. Neurology. 2011;76:1872-1878.

17. Longstreth W, Dulberg C, Manolio TA, Lewis MR, Beauchamp NJ, O'Leary D, Carr J, et al. Incidence, manifestations, and predictors of brain infarcts defined by serial cranial magnetic resonance imaging in the elderly: The Cardiovascular Health Study. Stroke. 2002;33:2376-2382.

18. Liem M, Oberstein SL, Haan J, van der Neut I, Ferrari M, van Buchem M, Middelkoop H, et al. MRI correlates of cognitive decline in CADASIL: A 7-year follow-up study. Neurology. 2009;72:143-148.

19. Veglio F, Paglieri C, Rabbia F, Bisbocci D, Bergui M, Cerrato P. Hypertension and cerebrovascular damage. Atherosclerosis. 2009;205:331-341.

20. Verhaaren BF, Vernooij MW, de Boer R, Hofman A, Niessen WJ, van der Lugt A, Ikram MA. High blood pressure and cerebral white matter lesion progression in the general population. Hypertension. 2013;61:1354-1359.

21. Henskens LH, van Oostenbrugge RJ, Kroon AA, de Leeuw PW, Lodder J. Brain microbleeds are associated with ambulatory blood pressure levels in a hypertensive population. Hypertension. 2008;51:62-68.

22. Huijts M, Duits A, van Oostenbrugge RJ, Kroon AA, de Leeuw PW, Staals J. Accumulation of MRI markers of cerebral small vessel disease is associated with decreased cognitive function: A study in first-ever lacunar stroke and hypertensive patients. Front Aging Neurosci. 2013;5:72.

23. De Jong G, Kessels F, Lodder J. Two types of lacunar infarcts further arguments from a study on prognosis. Stroke. 2002;33:2072-2076.

24. Verhage F. Intelligentie en leeftijd: onderzoek bij Nederlanders van twaalf tot zevenenzeventig jaar. Assen: Van Gorcum 1964.

25. Fazekas F, Chawluk JB, Alavi A, Hurtig HI, Zimmerman RA. MR signal abnormalities at 1.5 T in Alzheimer's dementia and normal aging. AJR Am J Roentgenol. 1987;149:351-356.

26. Wardlaw JM, Smith EE, Biessels GJ, Cordonnier C, Fazekas F, Frayne R, Lindley RI, et al. Neuroimaging standards for research into small vessel disease and its contribution to ageing and neurodegeneration. Lancet Neurol. 2013;12:822-838

27. Prins N, van Straaten E, van Dijk E, Simoni M, van Schijndel R, Vrooman H, Koudstaal P, et al. Measuring progression of cerebral white matter lesions on MRI Visual rating and volumetrics. Neurology. 2004;62:1533-1539.

28. Uiterwijk R, Huijts M, Staals J, Duits A, Gronenschild E, Kroon AA, de Leeuw PW, et al. Subjective cognitive failures in patients with hypertension are related to cognitive performance and cerebral microbleeds. Hypertension. 2014;64:653-657.

29. Golden CJ. Stroop Colour and Word Test. Chicago (IL): Stoelting; 1978.

30. Reitan R. Trail Making Test: Manual for administration, scoring and interpretation. Bloomington: Indiana University; 1956.

31. Luteyn F. Een nieuwe verkorte GIT. Dutch J Psychol. 1966;2:675-682.

32. Lezak MD, Howieson DB, Loring DW. Neuropsychological assessment. New York: Oxford University Press; 2004.

33. Wechsler D. WAIS III, nederlandstalige bewerking: technische handleiding. Lisse: Swets Test Publishers; 2001.

34. Brand N, Jolles J. Learning and retrieval rate of words presented auditorily and visually. J Gen Psychol. 1985;112:201-210.

35. De Koning I, van Kooten F, Koudstaal PJ, Dippel DW. Diagnostic value of the Rotterdam-CAMCOG in post-stroke 
dementia. J Neurol Neurosurg Psychiatry. 2005;76:263-265.

36. Spinhoven PH, Ormel J, Sloekers PP, Kempen GIJ, Speckens AE, van Hemert AM. A validation study of the Hospital Anxiety and Depression Scale (HADS) in different groups of Dutch subjects. Psychol Med. 1997;27:363-370.

37. Dufouil C, Chalmers J, Coskun O, Besancon V, Bousser M-G, Guillon P, Macmahon S, et al. Effects of blood pressure lowering on cerebral white matter hyperintensities in patients with stroke the PROGRESS (Perindopril Protection Against Recurrent Stroke Study) magnetic resonance imaging substudy. Circulation. 2005;112:16441650.

38. Firbank MJ, Wiseman RM, Burton EJ, Saxby BK, O'Brien JT, Ford GA. Brain atrophy and white matter hyperintensity change in older adults and relationship to blood pressure. J Neurol. 2007;254:713-721.

39. Henskens LH, Kroon AA, van Oostenbrugge RJ, Gronenschild EH, Hofman PA, Lodder J, de Leeuw PW. Associations of ambulatory blood pressure levels with white matter hyperintensity volumes in hypertensive patients. J Hypertens. 2009;27:1446-1452. 


\section{SUPPLEMENTARY MATERIAL}

Table S1. Associations between periventricular WMH and cognitive decline in cognitive domains

\begin{tabular}{llllll}
\hline & & $B$ & $95 \% \mathrm{Cl}$ & $p$ & $\boldsymbol{R}^{2}$ change \\
\hline Executive functioning* & Baseline MRI & 0.24 & -0.03 to 0.50 & 0.077 & $2.1 \%$ \\
& Progression MRI & 0.29 & 0.10 to 0.49 & 0.004 & $5.6 \%$ \\
\multirow{2}{*}{ Information processing speed +} & Baseline MRI & 0.23 & -0.13 to 0.58 & 0.210 & $1.1 \%$ \\
& Progression MRI & 0.26 & -0.01 to 0.53 & 0.060 & $2.5 \%$ \\
Memory $\neq$ & Baseline MRI & -0.10 & -0.40 to 0.20 & 0.497 & $0.3 \%$ \\
& Progression MRI & 0.20 & -0.02 to 0.42 & 0.080 & $1.7 \%$ \\
\hline
\end{tabular}

Corrected for age, sex, educational level, HADS total score, patient group, baseline cognition, and potential vascular risk factors

* Total $R^{2}$ of the model $=23.5 \%$

† Total $R^{2}$ of the model $=17.8 \%$

¥ Total $R^{2}$ of the model $=37.6 \%$

Table S2. Associations between subcortical WMH and cognitive decline in cognitive domains

\begin{tabular}{llllll}
\hline & & $\boldsymbol{B}$ & $95 \% \mathrm{Cl}$ & $\boldsymbol{p}$ & $\boldsymbol{R}^{2}$ change \\
\hline Executive functioning* & Baseline MRI & 0.49 & 0.18 to 0.80 & 0.002 & $6.7 \%$ \\
& Progression MRI & 0.02 & -0.17 to 0.20 & 0.876 & $0.0 \%$ \\
Information processing speed $\dagger$ & Baseline MRI & 0.40 & -0.02 to 0.82 & 0.061 & $2.6 \%$ \\
& Progression MRI & -0.11 & -0.36 to 0.14 & 0.401 & $0.5 \%$ \\
Memory $\ddagger$ & Baseline MRI & -0.07 & -0.42 to 0.28 & 0.695 & $0.1 \%$ \\
& Progression MRI & 0.11 & -0.10 to 0.31 & 0.316 & $0.6 \%$ \\
\hline
\end{tabular}

Corrected for age, sex, educational level, HADS total score, patient group, baseline cognition, and potential vascular risk factors

* Total $R^{2}$ of the model $=21.3 \%$

† Total $R^{2}$ of the model $=16.0 \%$

‡ Total $R^{2}$ of the model $=36.4 \%$ 
Table S3. Associations between microbleeds and cognitive decline in cognitive domains

\begin{tabular}{llllll}
\hline & & $B$ & $95 \% \mathrm{Cl}$ & $p$ & $\boldsymbol{R}^{2}$ change \\
\hline Executive functioning* & Baseline MRI & 0.09 & -0.14 to 0.33 & 0.432 & $0.4 \%$ \\
& Progression MRI & 0.20 & -0.11 to 0.50 & 0.203 & $1.2 \%$ \\
Information processing speed $t+$ & Baseline MRI & 0.20 & -0.11 to 0.51 & 0.198 & $1.2 \%$ \\
& Progression MRI & 0.34 & -0.05 to 0.73 & 0.090 & $2.1 \%$ \\
Memory $\neq$ & Baseline MRI & 0.15 & -0.11 to 0.41 & 0.253 & $0.7 \%$ \\
& Progression MRI & -0.12 & -0.47 to 0.22 & 0.485 & $0.3 \%$ \\
\hline
\end{tabular}

Corrected for age, sex, educational level, HADS total score, patient group, baseline cognition, and potential vascular risk factors

* Total $R^{2}$ of the model $=16.1 \%$

† Total $R^{2}$ of the model $=17.4 \%$

$\neq$ Total $R^{2}$ of the model $=36.7 \%$

Table S4. Associations between lacunes and cognitive decline in cognitive domains

\begin{tabular}{llllll}
\hline & & $B$ & $95 \% \mathrm{Cl}$ & $p$ & $\boldsymbol{R}^{2}$ change \\
\hline Executive functioning* & Baseline MRI & 0.22 & 0.02 to 0.41 & 0.031 & $3.3 \%$ \\
& Progression MRI & 0.04 & -0.25 to 0.32 & 0.812 & $0.0 \%$ \\
\multirow{2}{*}{ Information processing speed $t+$} & Baseline MRI & -0.04 & -0.30 to 0.22 & 0.758 & $0.1 \%$ \\
& Progression MRI & 0.29 & -0.09 to 0.68 & 0.134 & $1.7 \%$ \\
Memory $\ddagger$ & Baseline MRI & 0.10 & -0.11 to 0.31 & 0.361 & $0.5 \%$ \\
& Progression MRI & 0.15 & -0.17 to 0.46 & 0.357 & $0.5 \%$ \\
\hline
\end{tabular}

Corrected for age, sex, educational level, HADS total score, patient group, baseline cognition, and potential vascular risk factors

* Total $R^{2}$ of the model $=17.7 \%$

$\dagger$ Total $R^{2}$ of the model $=14.2 \%$

$\ddagger$ Total $R^{2}$ of the model $=36.9 \%$ 



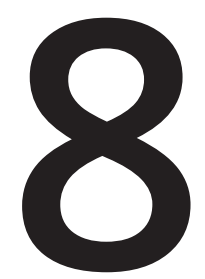

General discussion 

The general aim of this thesis was to determine predictors of cognitive function, cognitive decline and cerebral small vessel disease (CSVD)-related brain damage in patients with essential hypertension. In this chapter, we discuss the main findings of this thesis and the implications for clinical practice and future research. Three important questions will be addressed:

I. How can we predict cognitive function, cognitive decline and cSVD-related brain damage in patients with hypertension in clinical practice?

II. How do we need to study the prevention of cognitive decline and dementia in patients with hypertension in future research?

III. Can new imaging techniques improve studying hypertensive damage of the brain?

\section{How to predict cognitive function, cognitive decline and cSVD-related brain damage in patients with hypertension}

Hypertension is an important risk factor for lower cognitive function, which eventually can lead to vascular dementia. ${ }^{1}$ This is probably caused by structural brain damage due to cSVD. Current guidelines of the European Society of Hypertension and the European Society of Cardiology (ESH/ESC) recommend routine assessment of other sites of hypertensive organ damage, namely of the heart and kidneys, in the initial evaluation of all hypertensive patients. ${ }^{2}$ Although the brain is regarded as one of the major target organs, routine assessment of brain damage is not part of those guidelines. This is mostly related to a low availability and high cost of magnetic resonance imaging (MRI). ${ }^{2}$ More insight into predictors of CSVD-related brain damage or lower cognitive function in essential hypertension could help to identify high-risk patients who might be in need of further examination.

Our results demonstrated that subjective cognitive failures are independently associated with lower cognitive function and cerebral microbleeds (Chapter 2). Subjective cognitive failures are personal experiences of cognitive difficulties in everyday life and are easy to assess by interview or questionnaire. While it might seem obvious that these subjective cognitive problems are indicative of lower objectively measured cognition, most previous research performed in other populations, did not succeed in identifying this relation. ${ }^{3-8}$ An inquiry into cognitive difficulties in everyday life should be part of the anamnesis taken during the first contact. This is easy to implement in routine clinical practice and could be a first step in identifying patients with hypertension, who are at high risk of cognitive dysfunction and brain damage and may be in need of further examination.

The use of vascular risk scores might be another way to identify patients at high risk. Several vascular risk factors have been associated with lower cognitive function ${ }^{9-11}$ and CSVD-related brain damage, ${ }^{12-15}$ also independently of hypertension..$^{12,15}$ As risk factors often occur 
together, vascular risk scores have been composed to join multiple risk factors. The Framingham stroke risk profile (FSRP) is a well-known vascular risk score that is designed to predict 10-year risk of clinical stroke. ${ }^{16,17}$ We showed that the FSRP is associated with future lower cognitive performance and with progression of periventricular white matter hyperintensities $(\mathrm{WMH})$ and new microbleeds over 9 years (Chapter 4). Although this score has not been specifically designed to predict cSVD-related brain damage and future cognitive function, our results demonstrate that a vascular risk score might be useful for this purpose.

A multimodal risk score incorporating several of these predictors of cognitive impairment or CSVD-related brain damage could help to optimally identify those at high risk and to inform patients about risk factors and their own risk level. We established that a vascular risk score is predictive of cognitive decline and brain damage, but such an existing vascular risk score could be improved for this purpose. The vascular risk factors of the FSRP should be included in a multimodal risk score for predicting cognitive problems and brain damage in patients with hypertension. A few studies developed multimodal risk scores for prediction of dementia. The most well-known example is the Cardiovascular Risk Factors, Aging, and Dementia (CAIDE) study, that developed a dementia risk score in a middle-aged general population. ${ }^{18}$ The Australian National University Alzheimer's Disease Risk Index (ANU-ADRI) ${ }^{19}$ was developed specifically for predicting Alzheimer's Disease. It is likely that risk scores have diverging predictive values depending on the population they are developed in and used for, and the type of cognitive impairment they aim to predict. As such, a multimodal risk score to specifically predict cognitive impairment and decline in patients with hypertension is urgently needed. Vascular risk factors are expected to be more important in such a score compared to an Alzheimer's Disease risk score and therefore might receive a higher load. As we showed that subjective cognitive failures are associated with lower cognitive function in patients with hypertension, this could be another factor to be included in such a multimodal cognitive impairment risk score for hypertensive patients. In addition, it is known that premorbid cognitive function is an important predictor of cognitive impairment. As such, the score on the Dutch or national adult reading test, as a measure of premorbid cognition, could be included in the multimodal risk score.

Such a multimodal risk score could determine who is at risk of cognitive problems or cSVD-related brain damage. For those high-risk patients it is recommended to perform further examination, such as a brain MRI scan. Studies have shown that the presence of individual MRI markers, such as WMH and lacunes, are predictive of cognitive decline.20-22 However, in clinical practice we are often faced with the occurrence of different MRI markers of CSVD combined in one patient and it is unclear what the total CSVD-related brain damage implicates for a patient. Our study showed that a total cSVD score, composed of the presence of four MRI markers of CSVD, ${ }^{23}$ was associated with decline in cognitive func- 
tion over 4 years (Chapter 6). The total cSVD score is an easily applicable score in clinical practice when visually rating conventional MRI images. Clinicians might use the cSVD score to determine if a patient with hypertension is at higher risk of future cognitive decline. Further study of the CSVD score is needed to examine if the predictive value of the current cSVD score could be improved. For example, instead of dichotomizing every MRI marker, it might be better to attribute different points in the CSVD score for different degrees of damage. It may also be useful to include other markers of brain damage in the score. For example, cerebral atrophy is sometimes considered a marker of CSVD, although it is not specific for cSVD. Cerebral atrophy has also been associated with cognitive decline. ${ }^{24}$ Further research should determine if including other markers or refining the range of the cSVD score improves the prediction of cognitive decline.

\section{Future research on preventing cognitive decline in hypertensive patients}

One of the most important clinical questions is how to prevent cognitive decline or development of vascular cognitive impairment (VCI) in patients with hypertension. $\mathrm{VCl}$ is an umbrella term covering all cognitive deficits due to or related to cerebrovascular disease and is a slowly progressive syndrome. Therefore, it is crucial to identify factors that are responsible for the development of mild cognitive impairment and progression to dementia. The most common subtype of VCI is related to cSVD. ${ }^{25}$ Only few studies have investigated the association between progression of CSVD and cognitive decline and it is unclear whether baseline or progression of CSVD-related MRI markers is more important in predicting cognitive decline. Our results showed that progression of periventricular WMH was associated with cognitive decline over 4 years, while we could not establish an association for baseline presence of periventricular WMH and cognitive decline (Chapter 7). We showed in a multivariable analysis that baseline presence of periventricular WMH explained only $0.6 \%$ of variance in cognitive decline, while the progression of periventricular $\mathrm{WMH}$ explained $5.6 \%$. This might seem a rather small indicator, but it could be regarded a relevant effect compared to $18.3 \%$ of variance explained by all other covariates in the model (age, sex, educational level, anxiety and depression symptoms, and patient group). Preventing progression of periventricular WMH might thus be an option to prevent cognitive decline in hypertension. Studies in patients with and without cerebrovascular disease have shown that active blood pressure lowering might stop or delay the progression of $\mathrm{WMH}^{26,27}$ Although this emphasizes the importance of good blood pressure control in patients with hypertension, it does not provide direct evidence that this can also prevent cognitive decline. Research that directly investigates the effect of antihypertensive therapy on preventing cognitive decline or dementia in patients with hypertension is scarce and urgently needed.

A few randomized controlled trials (RCTs) have been performed to examine the effect of blood pressure lowering treatment on the prevention of dementia and cognitive decline. 
Two large studies, namely the Syst-Eur and the PROGRESS trials, did show an effect. The Syst-Eur showed that in an elderly population, active blood pressure treatment reduced the incidence of dementia by $50 \%$ compared to placebo group with a median follow-up of 2.0 years. ${ }^{28}$ The PROGRESS, a study of antihypertensive treatment in patients with stroke or transient ischemic attack and a mean follow-up of 3.9 years, reported no significant difference in incidence of dementia, but showed a difference in incidence of cognitive decline. ${ }^{29}$ However, several other RCTs did not report an effect in intention-to-treat analysis. ${ }^{30}$ A Cochrane review examined all RCTs on the effect of blood pressure treatments for the prevention of dementia and cognitive decline in patients with hypertension but without history of cerebrovascular disease. ${ }^{31}$ Based on the combined results of the SystEur trial and three other RCTs, the review concluded that these studies have not led to convincing evidence that blood pressure lowering in late life prevents the development of dementia or cognitive impairment. ${ }^{31}$

In the Systolic Blood Pressure Intervention Trial (SPRINT), 9361 patients with a SBP of $130 \mathrm{~mm} \mathrm{Hg}$ or higher and an increased cardiovascular risk were randomized to intensive treatment (SBP target $<120 \mathrm{~mm} \mathrm{Hg}$ ) or standard treatment (SBP target $<140 \mathrm{~mm}$ $\mathrm{Hg}){ }^{32}$ Results of the primary analysis showed a lower rate of a composite cardiovascular outcome in the intensive treatment group compared to the standard treatment group. Results on incident dementia, cognitive decline and CSVD have not been presented yet, but could be promising.

The negative results of most RCTs might be induced by multiple issues these studies encounter. First, the number of patients who develop dementia over time is rather low. This is related to a relatively short follow-up time in most studies, since it is known that development of dementia or cognitive impairment is a rather slow process. However, studies with a long follow-up are hard to carry out and often lead to a high dropout rate. Second, many studies have investigated the development of dementia or cognitive decline as a secondary outcome. For this reason, these studies might be statistically underpowered to draw any conclusions in regard to the effect of blood pressure lowering treatment on the prevention of dementia and cognitive decline. Third, in many studies cognitive decline is determined with the Mini-Mental State Examination (MMSE). The MMSE is only a screening method and makes it hard to find change over time. In addition, the MMSE does insufficiently assess executive function or information processing speed, ${ }^{33}$ while it is known that these cognitive functions are mostly affected by hypertension. ${ }^{34}$ We showed that the effects of MRI markers of CSVD on cognitive decline differ across cognitive domains. For example, we demonstrated that the total CSVD score was associated with cognitive decline in executive function, but not with decline in information processing speed or memory (Chapter 6). Therefore, it is possible that cognitive function in the domain of 
executive function might benefit more from blood pressure control than cognitive function in other domains. Fourth, evidence is most strong about associations between mid-life hypertension and late-life dementia, while the evidence about late-life hypertension and cognitive decline or dementia is less conclusive. ${ }^{35}$ However, most RCTs on the prevention of dementia included older participants and it is possible that the effect of antihypertensive therapy in these cohorts is reduced. The optimal window of intervention is hard to determine in dementia prevention trials, since the incidence of dementia increases with age while the effect of intervention decreases with age. ${ }^{36} \mathrm{Fifth}$, many factors play a role in cognitive function or development of dementia. We showed that several important predictors together, including age and progression of periventricular $\mathrm{WMH}$, explained only a quarter of variance in cognitive decline, so a large part of the variance was left unexplained (Chapter 7). This means that the effect of blood pressure on cognition or the development of dementia, and the effect of blood pressure treatment, will probably also be limited. Lastly, most RCTs used relatively healthy hypertension populations to study cognitive impairment. Studying a population with patients who are at higher risk might increase the incidence of dementia during follow-up. Since results of our study indicated that patients with a higher CSVD score at baseline have a steeper decline in cognitive function over 4 years (Chapter 6), this MRI cSVD score could be used to identify patients at higher risk of cognitive decline for the inclusion in RCTs on antihypertensive medication. A multimodal risk score for cognitive impairment, as previously suggested, could help to identify patients at higher risk for inclusion in treatment studies as well.

In conclusion, more research on prevention of cognitive decline in patients with hypertension is needed. A well-performed RCT to investigate the effect of different antihypertensive medications with cognitive decline and incident dementia as the primary outcomes is needed. It is important that such an RCT includes an extensive neuropsychological assessment covering different domains. Inclusion could be focused on patients at risk, identified by MRI CSVD score or a multimodal risk score. Despite practical difficulties, an RCT with long-term follow-up time and baseline starting in mid-life would be optimal.

\section{New techniques for studying hypertensive organ damage of the brain}

Evidence is now convincing that hypertension is not only a risk factor for end-organ damage of the kidneys and heart, ${ }^{37-40}$ but also of the brain. ${ }^{34}$ These organ damages might be regarded as markers of the severity of hypertensive disease. We demonstrated that an increasing number of hypertensive organ damages is associated with lower cognitive performance. In addition, cardiac damage was associated with lower cognitive performance independently of CSVD, while we could not show this for renal damage (Chapter 5). These results show that in patients with hypertensive cardiac damage, there should be an increased awareness for cognitive consequences. In addition, it suggests that cardiac 
damage might be a marker for more severe hypertensive disease burden. It might also be that cardiac pathology affects the hemodynamics of the brain, even before brain damage becomes visible. However, little is known about this relationship between heart and brain. The currently running Dutch Heart Brain Connection study aims to shed more light on this relationship. ${ }^{41}$

Although CSVD-related brain damage might be a marker of the severity of hypertensive disease, the best way to determine the hypertensive organ damage of the brain is not clear. Not all effects of hypertensive brain damage are visible on MRI. Hypertensive brain damage is also associated with microstructural brain damage and cerebrovascular functional alterations, which cannot be seen on conventional MRI. Other techniques might help to improve the identification of hypertensive brain damage. One possible improvement to conventional MRI might be Diffusion Tensor Imaging (DTI), which has been increasingly used in the last decade. With DTI, disturbance of microstructural integrity can be shown. It has proven to be more sensitive for white matter damage as it shows abnormalities not only in WMH but also in apparently normal appearing white matter (NAWM). ${ }^{42,43}$ Decreased microstructural integrity of NAWM and WMH have been shown to be associated with higher blood pressure levels. ${ }^{44}$ In addition, DTI studies showed that lower microstructural integrity in the white matter was associated with lower cognition.45-47

The use of high-field MRI techniques might offer new opportunities, as 7 Tesla (7T) MRI has recently become available for use in humans. Using $7 \mathrm{~T} \mathrm{MRI,} \mathrm{high} \mathrm{resolution} \mathrm{anatom-}$ ical imaging can be obtained, which provides more information about the parenchymal changes caused by CSVD. ${ }^{48}$ In addition, with 7T MR angiography it might be possible to visualize small vessels without using contrast agents. ${ }^{49-51}$ Besides a more detailed visualization of structural changes, 7T MRI might also enable the opportunity to show functional changes, such as blood flow velocity and pulsatility in the small vessels. ${ }^{52}$ Although 7T MRI is unlikely to replace 3T MRI in clinical practice, the use of 7T MRI in research offers more information on hypertensive brain damage, both at a structural and a functional level.

Another new approach is imaging of blood-brain barrier (BBB) permeability. Blood-brain barrier (BBB) failure is suggested to be one of the main and early steps in the pathogenesis of CSVD. ${ }^{53}$ The BBB is a function of the brain vascular endothelium and endothelial dysfunction could in the end lead to cSVD and cognitive impairment. BBB dysfunction has also been suggested as a consequence of hypertension. ${ }^{34}$ We have shown that a compound score of blood markers of endothelial dysfunction was associated with lower cognitive performance in patients with hypertension (Chapter 3), so endothelial dysfunction might also play a role in the pathogenesis of cognitive problems. Imaging of BBB permeability, as a function of the endothelium, might enable the possibility of visualizing CSVD before brain 
damage and MRI markers become evident on conventional MRI. Studies investigating BBB permeability mostly used CSF/serum albumin ratio technique to quantify leakage, which is acknowledged to reflect BBB integrity accurately. ${ }^{54}$ These studies showed an increased BBB permeability in patients with vascular dementia and with increasing $\mathrm{WMH} .{ }^{54}$ However, this technique does not allow investigation of regional differences in BBB breakdown. More recently, contrast-enhanced MRI has been developed to investigate BBB permeability. Few studies have used this technique in CSVD patients. One study showed an increased BBB permeability in the white matter of patients with CSVD. ${ }^{55}$ Another study showed that patients with lacunar stroke have a diffuse background increase in BBB permeability in the white matter, compared to cortical stroke patients. ${ }^{56} \mathrm{~A}$ third study reported that patients with CSVD show a larger volume of BBB leakage in the NAWM, cortical grey matter and WMH, compared to healthy controls. ${ }^{57}$ Future longitudinal studies should provide more insight in the use of contrast-enhanced MRI and the role of BBB dysfunction in the pathogenesis of cSVD and cognitive dysfunction. In addition, as studies that have investigated BBB permeability in hypertensive patients with contrast-enhanced MRI could not be found, future studies using new imaging techniques should investigate the influence of blood pressure on BBB integrity and its effect on cognition.

\section{Conclusion}

In conclusion, we have indicated several factors that could be used in predicting cognitive dysfunction or cognitive decline in patients with hypertension. It is important to use such predictors, ideally in a multimodal score, to identify high-risk patients and consider further examination of these patients, such as a neuropsychological assessment and brain imaging. More research is urgently needed to improve the prevention of cognitive decline in hypertensive patients, including well-performed RCTs with antihypertensive medications. Lastly, new imaging techniques could improve the detection of hypertensive brain damage, which should be used in further research on the implications of combined and independent hypertensive organ damages and the consequences of such brain damage for cognitive function. 


\section{REFERENCES}

1. Iadecola C. Hypertension and dementia. Hypertension. 2014;64:3-5.

2. Mancia G, Fagard R, Narkiewicz K, Redon J, Zanchetti A, Böhm M, Christiaens T, et al. 2013 ESH/ESC Guidelines for the management of arterial hypertension. Blood Press. 2013;22:193-278.

3. Haley AP, Hoth KF, Gunstad J, Paul RH, Jefferson AL, Tate DF, Ono M, et al. Subjective cognitive complaints relate to white matter hyperintensities and future cognitive decline in patients with cardiovascular disease. Am J Geriatr Psychiatry. 2009;17:976-985.

4. Xiong Y-Y, Wong A, Mok VC, Tang W-K, Lam WW, Kwok TC, Chu WC, et al. Frequency and predictors of proxy-confirmed post-stroke cognitive complaints in lacunar stroke patients without major depression. Int J Geriatr Psychiatry. 2011;26:1144-1151.

5. Lamb F, Anderson J, Saling M, Dewey H. Predictors of subjective cognitive complaint in post-acute older adult stroke patients. Arch Phys Med Rehabil. 2013;94:1747-1752.

6. Mascherek A, Zimprich D, Rupprecht R, Lang FR. What do cognitive complaints in a sample of memory clinic outpatients reflect? GeroPsych. 2011;24:187-195.

7. Duits A, Munnecom T, van Heugten C, van Oostenbrugge RJ. Cognitive complaints in the early phase after stroke are not indicative of cognitive impairment. J Neurol Neurosurg Psychiatry. 2008;79:143-146.

8. Jorm AF, Butterworth P, Anstey KJ, Christensen H, Easteal S, Maller J, Mather KA, et al. Memory complaints in a community sample aged 60-64 years: associations with cognitive functioning, psychiatric symptoms, medical conditions, APOE genotype, hippocampus and amygdala volumes, and white-matter hyperintensities. Psychol Med. 2004;34:1495-1506.

9. Nash DT, Fillit H. Cardiovascular disease risk factors and cognitive impairment. Am J Cardiol. 2006;97:12621265.

10. North T-L, Palmer TM, Lewis SJ, Cooper R, Power C, Pattie A, Starr JM, et al. Effect of smoking on physical and cognitive capability in later life: a multicohort study using observational and genetic approaches. BMJ open. 2015;5:e008393.

11. Baumgart M, Snyder HM, Carrillo MC, Fazio S, Kim H, Johns H. Summary of the evidence on modifiable risk factors for cognitive decline and dementia: A population-based perspective. Alzheimers Dement. 2015;11:718 726.

12. Gouw AA, van der Flier WM, Fazekas F, van Straaten EC, Pantoni L, Poggesi A, Inzitari D, et al. Progression of white matter hyperintensities and incidence of new lacunes over a 3-year period: The Leukoaraiosis and Disability Study. Stroke. 2008;39:1414-1420.

13. Wardlaw JM, Smith C, Dichgans M. Mechanisms of sporadic cerebral small vessel disease: insights from neuroimaging. Lancet Neurol. 2013;12:483-497.

14. Vermeer SE, Longstreth WT, Koudstaal PJ. Silent brain infarcts: a systematic review. Lancet Neurol. 2007;6:611619.

15. Van Dijk EJ, Prins ND, Vrooman HA, Hofman A, Koudstaal PJ, Breteler MM. Progression of cerebral small vessel disease in relation to risk factors and cognitive consequences: Rotterdam Scan Study. Stroke. 2008;39:27122719. 
16. D'Agostino RB, Wolf PA, Belanger AJ, Kannel WB. Stroke risk profile: adjustment for antihypertensive medication. The Framingham Study. Stroke. 1994;25:40-43.

17. Wolf PA, D'Agostino RB, Belanger AJ, Kannel WB. Probability of stroke: a risk profile from the Framingham Study. Stroke. 1991;22:312-318.

18. Kivipelto M, Ngandu T, Laatikainen T, Winblad B, Soininen $H$, Tuomilehto J. Risk score for the prediction of dementia risk in 20 years among middle aged people: a longitudinal, population-based study. Lancet Neurol. 2006;5:735-741.

19. Anstey KJ, Cherbuin N, Herath PM. Development of a new method for assessing global risk of Alzheimer's disease for use in population health approaches to prevention. Prev Sci. 2013;14:411-421.

20. Prins ND, van Dijk EJ, den Heijer T, Vermeer SE, Jolles J, Koudstaal PJ, Hofman A, et al. Cerebral small-vessel disease and decline in information processing speed, executive function and memory. Brain. 2005;128:20342041.

21. Jokinen H, Gouw A, Madureira S, Ylikoski R, van Straaten E, van Der Flier W, Barkhof F, et al. Incident lacunes influence cognitive decline: The LADIS study. Neurology. 2011;76:1872-1878.

22. Pavlovic AM, Pekmezovic T, Tomic G, Trajkovic JZ, Sternic N. Baseline predictors of cognitive decline in patients with cerebral small vessel disease. J Alzheimers Dis. 2014;42:S37-S43.

23. Staals J, Makin SD, Doubal FN, Dennis MS, Wardlaw JM. Stroke subtype, vascular risk factors, and total MRI brain small-vessel disease burden. Neurology. 2014;83:1228-1234.

24. Jokinen H, Lipsanen J, Schmidt R, Fazekas F, Gouw A, Van der Flier W, Barkhof F, et al. Brain atrophy accelerates cognitive decline in cerebral small vessel disease The LADIS study. Neurology. 2012;78:1785-1792.

25. Dichgans M, Leys D. Vascular cognitive impairment. Circ Res. 2017;120:573-591.

26. Dufouil C, Chalmers J, Coskun O, Besancon V, Bousser M-G, Guillon P, Macmahon S, et al. Effects of blood pressure lowering on cerebral white matter hyperintensities in patients with stroke the PROGRESS (Perindopril Protection Against Recurrent Stroke Study) magnetic resonance imaging substudy. Circulation. 2005;112:16441650.

27. Firbank MJ, Wiseman RM, Burton EJ, Saxby BK, O'Brien JT, Ford GA. Brain atrophy and white matter hyperintensity change in older adults and relationship to blood pressure. J Neurol. 2007;254:713-721.

28. Forette F, Seux M-L, Staessen JA, Thijs L, Birkenhäger WH, Babarskiene M-R, Babeanu S, et al. Prevention of dementia in randomised double-blind placebo-controlled Systolic Hypertension in Europe (Syst-Eur) trial. The Lancet. 1998;352:1347-1351.

29. The PROGRESS Collaborative Group, Tzourio C, Anderson C, Chapman N, Woodward M, Neal B, MacMahon S, et al. Effects of blood pressure lowering with perindopril and indapamide therapy on dementia and cognitive decline in patients with cerebrovascular disease. Arch Intern Med. 2003;163:1069-75.

30. Feigin $V$, Ratnasabapathy $Y$, Anderson C. Does blood pressure lowering treatment prevents dementia or cognitive decline in patients with cardiovascular and cerebrovascular disease? J Neurol Sci. 2005;229:151-155.

31. McGuinness B, Todd S, Passmore P, Bullock R. Blood pressure lowering in patients without prior cerebrovascular disease for prevention of cognitive impairment and dementia. The Cochrane Library. 2009.

32. The SPRINT Research Group, Wright JT, Williamson JD, Whelton PK, Snyder JK, Sink KM, Rocco MV, et al. A randomized trial of intensive versus standard blood-pressure control. N Eng/J Med. 2015:2103-2116. 
33. Webb AJ, Pendlebury ST, Li L, Simoni M, Lovett N, Mehta Z, Rothwell PM. Validation of the Montreal cognitive assessment versus mini-mental state examination against hypertension and hypertensive arteriopathy after transient ischemic attack or minor stroke. Stroke. 2014;45:3337-3342.

34. Iadecola C, Yaffe K, Biller J, Bratzke LC, Faraci FM, Gorelick PB, Gulati M, et al. Impact of hypertension on cognitive function: A scientific statement from the American Heart Association. Hypertension. 2016;68:e67-e94.

35. Hughes TM, Sink KM. Hypertension and its role in cognitive function: Current evidence and challenges for the future. Am J Hypertens. 2015;29:149-157.

36. Richard E, Andrieu S, Solomon A, Mangialasche F, Ahtiluoto S, van Charante EPM, Coley N, et al. Methodological challenges in designing dementia prevention trials—the European Dementia Prevention Initiative (EDPI). J Neurol Sci. 2012;322:64-70.

37. Lindroos M, Kupari M, Heikkila J, Tilvis R. Predictors of left ventricular mass in old age: an echocardiographic, clinical and biochemical investigation of a random population sample. Eur Heart J. 1994;15:769-780.

38. Levy D, Anderson MK, Savaga DD, Kannel BW, Christiansen CJ, Castelli PW. Echocardiographically detected left ventricular hypertrophy: Prevalence and risk factors. Ann Intern Med. 1988;108:7-13.

39. Wesson DE. Hypertension and the kidney. Curr Hypertens Rep. 2001;3:511-516.

40. Cerasola G, Mulè G, Cottone S, Nardi E, Cusimano P. Hypertension, microalbuminuria and renal dysfunction: The Renal Dysfunction in Hypertension (REDHY) study. J Nephrol. 2008;21:368-373.

41. Van Buchem MA, Biessels GJ, Brunner la Rocca HP, de Craen AJ, van der Flier WM, Ikram MA, Kappelle LJ, et al. The heart-brain connection: a multidisciplinary approach targeting a missing link in the pathophysiology of vascular cognitive impairment. J Alzheimers Dis. 2014;42:S443-S451.

42. O'Sullivan M, Summers PE, Jones DK, Jarosz JM, Williams SCR, Markus HS. Normal-appearing white matter in ischemic leukoaraiosis: A diffusion tensor MRI study. Neurology. 2001;57:2307-2310.

43. Pasi M, van Uden IW, Tuladhar AM, de Leeuw F-E, Pantoni L. White matter microstructural damage on diffusion tensor imaging in cerebral small vessel disease. Stroke. 2016;47:1679-1684.

44. Gons RA, de Laat KF, van Norden AG, van Oudheusden LJ, van Uden IW, Norris DG, Zwiers MP, et al. Hypertension and cerebral diffusion tensor imaging in small vessel disease. Stroke. 2010;41:2801-2806.

45. Tuladhar AM, van Norden AG, de Laat KF, Zwiers MP, van Dijk EJ, Norris DG, de Leeuw F-E. White matter integrity in small vessel disease is related to cognition. Neuroimage Clin. 2015;7:518-524.

46. Van Norden AG, de Laat KF, van Dijk EJ, van Uden IW, van Oudheusden LJ, Gons RA, Norris DG, et al. Diffusion tensor imaging and cognition in cerebral small vessel disease: the RUN DMC study. Biochim Biophys Acta. 2012;1822:401-407.

47. Patel B, Markus HS. Magnetic resonance imaging in cerebral small vessel disease and its use as a surrogate disease marker. Int J Stroke. 2011;6:47-59

48. Benjamin P, Viessmann O, MacKinnon AD, Jezzard P, Markus HS. 7 Tesla MRI in cerebral small vessel disease. Int J Stroke. 2015;10:659-664.

49. Cho Z-H, Kang C-K, Han J-Y, Kim S-H, Kim K-N, Hong S-M, Park C-W, et al. Observation of the lenticulostriate arteries in the human brain in vivo using 7.0 T MR angiography. Stroke. 2008;39:1604-1606.

50. Kang C-K, Park C-A, Lee H, Kim S-H, Park C-W, Kim Y-B, Cho Z-H. Hypertension correlates with lenticulostriate arteries visualized by 7T magnetic resonance angiography. Hypertension. 2009;54:1050-1056. 
51. Hendrikse J, Zwanenburg J, Visser F, Takahara T, Luijten P. Noninvasive depiction of the lenticulostriate arteries with time-of-flight MR angiography at 7.0 T. Cerebrovasc Dis. 2008;26:624-629.

52. Bouvy W, Geurts L, Kuijf H, Luijten P, Kappelle L, Biessels G, Zwanenburg J. Assessment of blood flow velocity and pulsatility in cerebral perforating arteries with 7-T quantitative flow MRI. NMR Biomed. 2015.

53. Wardlaw JM. Blood-brain barrier and cerebral small vessel disease. J Neurol Sci. 2010;299:66-71.

54. Farrall AJ, Wardlaw JM. Blood-brain barrier: ageing and microvascular disease - Systematic review and meta-analysis. Neurobiol Aging. 2009;30:337-352.

55. Topakian R, Barrick T, Howe F, Markus H. Blood-brain barrier permeability is increased in normal-appearing white matter in patients with lacunar stroke and leucoaraiosis. J Neuro/ Neurosurg Psychiatry. 2010;81:192-197.

56. Wardlaw JM, Doubal F, Armitage P, Chappell F, Carpenter T, Muñoz Maniega S, Farrall A, et al. Lacunar stroke is associated with diffuse blood-brain barrier dysfunction. Ann Neurol. 2009;65:194-202.

57. Zhang CE, Wong SM, van de Haar HJ, Staals J, Jansen JF, Jeukens CR, Hofman PA, et al. Blood-brain barrier leakage is more widespread in patients with cerebral small vessel disease. Neurology. 2017;88:426-432. 

Summary 

Hypertension is a leading cause of morbidity and mortality, because it results in damage in several end organs, such as the kidneys, heart and brain. Hypertension is, besides age, the most important risk factor for haemorrhagic and ischemic stroke. In addition, hypertension is strongly associated with cerebral small vessel disease (cSVD). The term cSVD is used to describe all pathological processes that affect the small vessels of the brain. As these small brain vessels are difficult to visualize in vivo by standard imaging methods, the lesions that occur in the brain parenchyma due to cSVD have been accepted as imaging markers of CSVD. These lesions are preferably visualized by Magnetic Resonance Imaging (MRI) and include white matter hyperintensities (WMH), lacunes, cerebral microbleeds and perivascular spaces.

Multiple studies have focused on the implications of MRI markers of CSVD for cognitive performance or impairment. Most of these studies had a cross-sectional design and evidence is most convincing for associations between MRI markers and global cognitive function and the domains of processing speed and executive function. Fewer studies had a longitudinal design, so assessed the relation between MRI markers and cognitive decline. These studies mostly focused on baseline presence of WMH and lacunes and showed associations with cognitive decline. Few longitudinal studies focused on progression of MRI markers over time and some of these studies showed associations between WMH progression and cognitive decline.

Although the evidence for a relationship between hypertension, CSVD and cognitive function, specifically for speed of mental processing and executive function, is quite strong, the degree of CSVD-related brain damage and cognitive problems greatly differs between hypertensive patients. This means that other factors also influence CSVD and cognitive function, or may moderate the relationship between hypertension, brain damage and cognitive function. More insight into predictors of cognitive performance and MRI markers of CSVD in hypertensive patients is important for identifying high-risk patients.

The general aim of this thesis was to determine predictors of cognitive function, cognitive decline and CSVD-related brain damage in patients with hypertension. We addressed the research objectives in a cohort of patients with essential hypertension who have been followed for a period of 9 years. For two research aims (chapter 6 and 7), a patient cohort with a broader spectrum of severity of cSVD was desired. Therefore, in addition to the patients with essential hypertension, we included hypertensive patients with a lacunar stroke.

In chapter 2 we investigated if subjective cognitive failures (SCF) are associated with objective cognitive function and/or MRI markers of CSVD in patients with hypertension. SCF are personal experiences of cognitive difficulties in everyday life and are easy to assess by 
interview or questionnaire. While it might seem obvious that these subjective cognitive problems are indicative of lower objectively measured cognition, most previous research performed in other populations could not find this. We showed that SCF are associated with lower cognitive function and cerebral microbleeds, independent of potential confounders such as depression and anxiety. As it is an easy action to ask for cognitive difficulties in everyday life, this might be a first step in identifying patients with hypertension who are at high risk and may be in need of further examination.

In chapter $\mathbf{3}$ we investigated if compound scores of endothelial activation or inflammation, based on several blood markers, are associated with future cognitive performance. Endothelial activation and inflammation have been suggested to play an important role in the pathogenesis of CSVD, but studies have been inconsistent on the association between individual blood markers and cognition. The use of a compound score of blood markers creates the possibility to draw more general conclusions about the underlying pathogenetic mechanisms leading to cognitive problems in hypertensive patients. We showed that a compound score of blood markers of endothelial activation, but not inflammation, was associated with lower cognitive performance three years later. This indicates that a process involving endothelial activation might play a role in the pathogenic pathways of cognitive problems in patients with hypertension.

The Framingham stroke risk profile (FSRP) is a well-known vascular risk score developed to predict clinical stroke in the individual. In chapter $\mathbf{4}$ we investigated if the FSRP at baseline is also associated with more 'silent' effects of cerebrovascular disease, namely with progression of MRI markers of CSVD and future cognitive performance. We showed that the FSRP was associated with progression of periventricular $\mathrm{WMH}$ and new microbleeds over 9 years. These associations lost significance after correction for the FSRP age component. In addition, the FSRP was associated with lower overall cognitive performance, which also applied to executive function, information processing speed and memory. For executive function, the association with FSRP lost significance after adjustment for the age component. Our results showed that the FSRP is associated with progressive CSVD and lower future cognitive performance in patients with hypertension. However, for progressive CSVD, age seems to be the most important component. These results support the importance of early detection of other vascular risk factors in patients with hypertension. Clinicians should be aware of the entire risk factor profile in attempting to preserve cognitive performance.

In chapter $\mathbf{5}$ we investigated the implications of cardiac and renal organ damage, independent of brain damage, and of the combined presence of these sites of hypertensive organ damage for future cognitive function. We showed that presence of 2 or 3 sites of 
organ damage compared to no organ damage was associated with lower cognitive performance 9 years later. In addition, we showed that cardiac damage, expressed as left ventricular mass index, was associated with future lower cognitive function independently of cSVD burden, while we could not show this association for renal damage. These results indicate that cardiac organ damage might be a marker of more severe hypertensive disease burden. In addition, it implicates that there should be awareness for cognitive function in patients with hypertensive cardiac organ damage.

Longitudinal studies have shown associations between baseline presence of MRI markers of CSVD and cognitive decline. However, most of these studies investigated individual MRI markers of CSVD, while in reality these MRI markers often co-occur in one patient. To capture the total CSVD-related brain damage, a total CSVD score has been developed, which integrates all individual MRI markers into one measure. This score was shown to be associated with lower cognitive performance in cross-sectional studies, but was not applied before in longitudinal research. Therefore, in chapter 6 we investigated if the total cSVD score is predictive of future cognitive decline in patients with hypertension. We showed that the cSVD score was associated with decline in cognitive function over 4 years, specifically in executive function. These results emphasize the importance of considering total brain damage due to CSVD, instead of looking at individual MRI markers.

Only few longitudinal studies have investigated the association between progression of MRI markers of CSVD and cognitive decline. In addition, it is unclear whether baseline presence or progression of CSVD is more predictive of cognitive decline. Therefore, in chapter 7 we investigated the association between progression of MRI markers of cSVD and cognitive decline over 4 years and compared this with the association between baseline presence of the MRI markers and cognitive decline. We showed that progression of periventricular WMH over 4 years was associated with cognitive decline, while we could not show an association between baseline periventricular WMH and cognitive decline. We did not find significant associations between baseline presence or progression of the other MRI markers and cognitive decline. These results emphasize the importance of preventing progression of periventricular WMH in hypertensive patients.

In chapter $\mathbf{8}$ we discussed the findings of this thesis and three important questions that emerged from our results and other existing studies. First, we addressed the question how we can predict cognitive function, cognitive decline and CSVD-related brain damage in patients with hypertension in clinical practice. We reported several factors that could be used to identify high-risk patients, including SCF, the FSRP and the MRI total CSVD score. Optimally, a compound risk score would be composed of these and other important predictors, to identify high-risk patients. Second, an important question is how we should 
study the prevention of cognitive decline and dementia in patients with hypertension. As previous randomized controlled trials (RCTs) are not conclusive about the effects of antihypertensive medications on preserving cognitive function, a well-performed RCT is needed. In such an RCT it is important that cognitive decline and incident dementia are the primary outcomes, in which these are determined with an extensive neuropsychological assessment covering different domains. In addition, as evidence is most conclusive about mid-life hypertension and late-life dementia, an RCT starting in mid-life with a long follow-up would be most likely to show positive results. Predictors of high risk of cognitive decline, such as the total CSVD score, could also be used as inclusion criteria for RCTs to increase the incidence of cognitive decline or dementia during follow-up. Lastly, we discussed new techniques for studying hypertensive organ damage of the brain. As conventional MRI cannot show microstructural brain damage and cerebrovascular functional alterations, other techniques might help to improve detection of hypertensive brain damage. These techniques include high-field MRI, diffusion tensor imaging and imaging of blood-brain barrier permeability. With these new imaging techniques, the detection of hypertensive brain damage could be improved, which should be used in further research to the consequences of such brain damage for cognitive function. 


Nederlandse samenvatting 

Hypertensie is een van de belangrijkste oorzaken van morbiditeit en mortaliteit, mede doordat het kan leiden tot schade aan verschillende organen, zoals nieren, hart en brein. Hypertensie is, na leeftijd, de belangrijkste risicofactor voor het optreden van hersenbloedingen en herseninfarcten. Daarnaast is hypertensie sterk geassocieerd met cerebral small vessel disease (ziekte van de kleine bloedvaten in de hersenen; cSVD). De term cSVD wordt gebruikt voor alle pathologische processen die de kleine vaten in het brein aantasten. Omdat deze kleine vaten in het brein moeilijk in vivo in beeld gebracht kunnen worden, zijn de laesies in het hersenweefsel die veroorzaakt worden door de pathologische processen in de kleine vaten geaccepteerd als "markers" van cSVD. Deze laesies kunnen zichtbaar gemaakt worden met behulp van beeldvormend onderzoek van de hersenen, het beste met Magnetic Resonance Imaging (MRI), en omvatten white matter hyperintensities (witte stof hyperintensiteiten; WMH), lacunes, cerebrale microbloedingen en perivasculaire ruimtes.

Meerdere studies hebben de gevolgen van MRI markers van cSVD voor cognitieve prestaties of beperkingen onderzocht. De meeste van deze studies hadden een cross-sectioneel design en gaven met name bewijs voor associaties tussen MRI markers en globaal cognitief functioneren en de cognitieve domeinen informatie verwerkingssnelheid en executief functioneren. Veel minder studies hebben longitudinaal onderzoek naar MRI markers en cognitieve achteruitgang uitgevoerd. Deze studies hebben zich met name gericht op baseline aanwezigheid van WMH en lacunes en vonden associaties met cognitieve achteruitgang. Slechts weinig longitudinaal onderzoek heeft de toename van MRI markers over tijd onderzocht, waarbij een aantal studies een relatie tussen WMH toename en cognitieve achteruitgang liet zien.

Ondanks redelijk sterk bewijs voor een relatie tussen hypertensie, cSVD en cognitief functioneren, met name voor informatie verwerkingssnelheid en executief functioneren, verschilt de mate van CSVD-gerelateerde hersenschade en cognitieve problemen sterk tussen patiënten met hypertensie. Dit betekent dat andere factoren het cognitief functioneren en de ontwikkeling van cSVD beïnvloeden, of de relatie tussen hypertensie, hersenschade en cognitie modereren. Meer inzicht in de voorspellers van cognitief functioneren en MRI markers van cSVD in patiënten met hypertensie is belangrijk om patiënten met een hoog risico te kunnen identificeren.

Het algemene doel van deze thesis was om voorspellers van cognitief functioneren, cognitieve achteruitgang en CSVD-gerelateerde hersenschade in patiënten met hypertensie te bepalen. Het onderzoek is uitgevoerd in een cohort van patiënten met essentiële hypertensie die gedurende 9 jaar gevolgd zijn. Voor twee onderzoeksdoelen (hoofdstuk 6 en 7) was een patiënten cohort met een breder spectrum van ernst van cSVD gewenst. 
Daarom hebben we hiervoor, naast de patiënten met essentiële hypertensie, een groep hypertensieve patiënten met een lacunaire beroerte geïncludeerd.

In hoofdstuk 2 hebben we onderzocht of subjective cognitive failures (subjectieve cognitieve vergissingen; SCF) geassocieerd zijn met objectief cognitief functioneren en/of MRI markers van CSVD in patiënten met hypertensie. SCF zijn persoonlijke ervaringen van cognitieve problemen in het dagelijkse leven en zijn makkelijk te bepalen in een gesprek of aan de hand van een vragenlijst. Terwijl het voor de hand lijkt te liggen dat deze subjectieve cognitieve problemen een indicatie zijn voor lagere objectief gemeten cognitie, kunnen de meeste eerdere studies in andere populaties deze relatie niet aantonen. Wij lieten zien dat in patiënten met hypertensie SCF geassocieerd zijn met lager cognitief functioneren en cerebrale microbloedingen, onafhankelijk van mogelijke confounders zoals depressie en angst. Aangezien het een makkelijke handeling is om te vragen naar het optreden van cognitieve problemen in het dagelijks leven, zou dit een eerste stap kunnen zijn in het identificeren van patiënten met hypertensie die een hoog risico op cognitieve problemen hebben en verder onderzoek nodig hebben.

In hoofdstuk 3 hebben wij onderzocht of samengestelde scores van endotheel activatie of inflammatie, gebaseerd op meerdere bloed markers, geassocieerd zijn met latere cognitieve prestaties. Een belangrijke rol is gesuggereerd voor endotheel activatie en inflammatie in de pathogenese van CSVD, maar studies zijn inconsistent over de associatie tussen individuele markers en cognitie. Het gebruik van een samengestelde score van bloed markers geeft de mogelijkheid om algemenere conclusies te trekken over de onderliggende pathogenese van cognitieve problemen in patiënten met hypertensie. We lieten zien dat een samengestelde score van bloed markers van endotheel activatie, maar niet van inflammatie, geassocieerd was met een lagere cognitieve prestatie drie jaar later. Dit laat zien dat een proces van endotheel activatie een rol zou kunnen spelen in de pathogene paden van cognitieve problemen in patiënten met hypertensie.

De Framingham stroke risk profile (Framingham beroerte risicoprofiel; FSRP) is een bekende vasculaire risicoscore die is ontwikkeld om de kans op een beroerte te voorspellen. In hoofdstuk 4 hebben we onderzocht of de FSRP ook geassocieerd is met 'stille' effecten van cerebrovasculaire ziekte, te weten met de progressie van MRI markers van CSVD en latere cognitieve prestaties. We lieten zien dat de FSRP gerelateerd was aan progressie van periventriculaire WMH en nieuwe microbloedingen over 9 jaar. Deze associaties waren niet meer significant na correctie voor de FSRP leeftijdscomponent. Daarnaast rapporteerden we een relatie tussen de FSRP en lagere overall cognitie en de domeinen executief functioneren, informatie verwerkingssnelheid en geheugen. Alleen voor executief functioneren verloor de associatie met FSRP significantie na correctie voor de leeftijdscomponent. Deze 
resultaten laten zien dat de FSRP geassocieerd is met progressieve CSVD en cognitieve prestatie in patiënten met hypertensie, al bleek de leeftijdscomponent het meest belangrijk voor progressieve cSVD. Deze resultaten ondersteunen het belang van vroege detectie van andere vasculaire risicofactoren in patiënten met hypertensie. Artsen zouden zich bewust moeten zijn van het totale vasculaire risicoprofiel om het cognitief functioneren van de patiënt met hypertensie te behouden.

In hoofdstuk 5 onderzochten we de implicaties van orgaanschade in hart en nieren, onafhankelijk van breinschade, en de gecombineerde aanwezigheid van deze plekken van hypertensieve orgaanschade voor later cognitief functioneren. We lieten zien dat de aanwezigheid van 2 of 3 plekken van orgaanschade in vergelijking tot geen orgaanschade, geassocieerd was met lagere cognitieve prestatie 9 jaar later. Daarnaast rapporteerden we dat hartschade, bepaald met de linker ventrikel massa index, geassocieerd was met lager cognitief functioneren 9 jaar later onafhankelijk van cSVD schade, terwijl we deze associatie niet konden aantonen voor nierschade. Deze resultaten impliceren dat orgaanschade in het hart een marker zou kunnen zijn voor een ernstigere vorm van hypertensieve ziekte. Daarnaast laten deze bevindingen zien dat er aandacht zou moeten zijn voor cognitief functioneren in patiënten met hypertensieve hartschade.

Longitudinale studies hebben een relatie aangetoond tussen baseline aanwezigheid van MRI markers van CSVD en cognitieve achteruitgang. Maar de meeste van deze studies onderzochten individuele MRI markers van CSVD, terwijl in werkelijkheid deze MRI markers vaak samen voorkomen bij een patiënt. Om de totale cSVD-gerelateerde breinschade te omvatten is er een totale CSVD score ontworpen die alle individuele MRI markers in één maat integreert. Eerdere cross-sectionele studies hebben laten zien dat deze totale cSVD score geassocieerd was met lagere cognitieve prestaties, maar deze score was nog niet eerder toegepast in longitudinaal onderzoek. Daarom hebben wij in hoofdstuk 6 onderzocht of een totale CSVD score voorspellend is voor cognitieve achteruitgang in patiënten met hypertensie. We lieten zien dat de CSVD score geassocieerd was met een achteruitgang in cognitief functioneren over 4 jaar, specifiek in het domein van executief functioneren. Deze resultaten benadrukken het belang van het beschouwen van de totale brein schade door CSVD in plaats van naar individuele MRI markers te kijken.

Slechts enkele longitudinale studies hebben de associatie tussen progressie van MRI markers van CSVD en cognitieve achteruitgang onderzocht en het is onduidelijk of baseline aanwezigheid of de progressie van CSVD belangrijker is in de voorspelling van cognitieve achteruitgang. Daarom onderzochten we in hoofdstuk 7 de associatie tussen progressie van MRI markers van cSVD en cognitieve achteruitgang over 4 jaar en vergeleken dit met de associatie tussen baseline aanwezigheid van de MRI markers en cognitieve achteruit- 
gang. We lieten zien dat een toename van periventriculaire WMH over 4 jaar geassocieerd is met een grotere cognitieve achteruitgang, terwijl we geen associatie konden aantonen tussen periventriculaire WMH op baseline en cognitieve achteruitgang. We vonden geen significante associaties tussen baseline aanwezigheid of progressie van de andere MRI markers en cognitieve achteruitgang. Deze resultaten benadrukken het belang om progressie van periventriculaire WMH in patiënten met hypertensie te voorkomen.

In hoofdstuk 8 bediscussieerden we de resultaten van deze thesis en drie belangrijke vragen die ontstonden naar aanleiding van de resultaten van onze studie en die van eerdere studies. Als eerste bekeken we de vraag hoe we cognitief functioneren, cognitieve achteruitgang en CSVD-gerelateerde breinschade in patiënten met hypertensie in de klinische praktijk kunnen voorspellen. We rapporteerden verschillende factoren die gebruikt kunnen worden in het identificeren van patiënten met hoog risico, waaronder SCF, de FSRP en de totale CSVD score. Een risicoscore zou samengesteld kunnen worden van deze en andere belangrijke voorspellers om zo patiënten met een hoog risico te identificeren. De tweede belangrijke vraag is hoe we de preventie van cognitieve achteruitgang en dementie in patiënten met hypertensie zouden moeten onderzoeken. Aangezien eerdere randomized controlled trials (gerandomiseerd vergelijkend onderzoeken; RCTs) niet overtuigend zijn geweest over de effecten van antihypertensiva voor het behouden van cognitief functioneren, is een goed uitgevoerde RCT nodig. Hierbij is het belangrijk dat cognitieve achteruitgang en ontwikkeling van dementie de primaire uitkomsten zijn, waarbij deze bepaald worden met een uitgebreid neuropsychologisch onderzoek dat meerdere domeinen omvat. Aangezien het bewijs het sterkst is voor de relatie tussen hypertensie op middelbare leeftijd en dementie op oudere leeftijd, zou een RCT die start tijdens middelbare leeftijd en een lange follow-up periode heeft de grootste kans hebben om positieve resultaten te vinden. Daarnaast zouden voorspellers van patienten met een hoog risico voor cognitieve achteruitgang, zoals bijvoorbeeld de totale cSVD score, gebruikt kunnen worden als inclusiecriteria van RCTs om hiermee het voorkomen van cognitieve achteruitgang of dementie tijdens de follow-up te verhogen. Als derde en laatste punt bediscussieerden we nieuwe technieken om hypertensieve schade in het brein te kunnen bestuderen. Conventionele MRI kan geen microstructurele breinschade en cerebrovasculaire functionele veranderingen aantonen. Nieuwe technieken zouden kunnen helpen om hypertensieve breinschade te visualiseren, zoals hoge veldsterkte MRI, diffusion tensor imaging en beeldvorming van bloed-hersenbarrière permeabiliteit. Met deze nieuwe beeldvormende technieken zou de detectie van hypertensieve breinschade verbeterd kunnen worden, dat vervolgens gebruikt zou kunnen worden in verder onderzoek naar de consequenties van zulke breinschade voor cognitief functioneren. 




\section{Valorisation}





\section{Introduction}

Knowledge valorisation refers to "the process of creating value from knowledge, by making knowledge suitable and/or available for social and/or economic use, and by making knowledge suitable for translation into competitive products, services, processes and new commercial activities" (adapted definition based on the National Valorisation Committee 2011:8). In other words, it is the important translation of scientific knowledge to clinical or societal relevance.

\section{Hypertension and the brain}

Hypertension is a highly prevalent condition and is one of the leading risk factors for global disease burden. It causes damage in several end organs, such as the kidneys, heart and eyes. Guidelines recommend to routinely assess hypertensive organ damage in the heart and kidneys. Brain damage is less recognized as hypertensive end-organ damage and is an under-researched area, even though the brain is one of the main target organs affected by hypertension. It is known that hypertension-related brain damage is mostly due to cerebral small vessel disease (CSVD). As a consequence, hypertension is a major risk factor for cognitive deficits or dementia. This thesis contributes to raising the attention for these under-recognized consequences of hypertension and increasing our knowledge about influencing factors and predictors. As it is not possible to perform a neuropsychological assessment or brain imaging in every hypertensive patient, it is important to find predictors for lower cognitive function or brain damage in patients with hypertension to identify those patients at risk and in need of further investigation. It is important that these predictors are easy to apply in clinical practice.

\section{Awareness for subjective cognitive failures in hypertensive patients}

Patients complaining about cognitive problems may be a recognizable situation for clinicians. Most previous studies have shown that these subjective problems, also called subjective cognitive failures (SCF), have a strong association with poor physical health and depression. Although it might seem obvious that these SCF would also relate to objective cognitive problems, most previous studies did not find this relationship when correcting for mood and health. However, this relationship had not been examined in patients with hypertension, even though there is increasing support for an association between hypertension and objective cognitive impairment. We showed that in patients with hypertension, SCF are associated with objectively measured cognitive function and presence of cerebral microbleeds.

Our results have important implications for clinical practice. Clinicians working with hypertensive patients should ask for the presence of cognitive problems during consultation of patients with hypertension, as these SCF may point to lower cognitive function and brain 
damage. It emphasizes the importance of performing a neuropsychological assessment and brain imaging when patients with hypertension report cognitive problems.

To increase awareness, it is important to inform both clinicians and patients. Therefore, in addition to a published scientific article, our results and the implications for clinical practice were described in the clinical implications section of Hypertension and were commented on in an editorial commentary in the same issue. ${ }^{2}$ In addition, a short summary of results was published on Dutch websites. ${ }^{3}$

\section{The total SVD score}

The different MRI markers of CSVD are generally investigated individually. However, in reality these markers might co-occur and it is thought that the occurrence of these markers together represents the severity of the underlying small vessel disease. Therefore, a "total SVD score" was recently proposed by our research group, which combines all individual MRI markers into one measure of SVD to capture total CSVD-related brain damage. This SVD score might provide a more complete overview of total SVD-related brain damage than just studying individual MRI markers.

In this thesis, we presented the first longitudinal study into the predictive value of the SVD score and we showed that the SVD score was associated with cognitive decline over 4 years. Our study contributes to the further validation and usefulness of the concept of a total score for cSVD. This total score uses visual rating of MRI and does not need computer software. This makes it an easy tool to use in clinical practice. Since the proposition of this total SVD score, interest has grown in such a total score and several other research groups have applied the score and showed that the SVD score is also associated with recurrent stroke and mortality. In addition, the concept of a total brain damage score has spread further. Other total scores have been proposed, such as a cerebrovascular disease burden score, which not only includes the presence of small vessel disease but also of large vessel disease, and a total MRI burden of SVD specifically adapted for patients with cerebral amyloid angiopathy. Further research should examine if the proposed SVD score could be improved, for example by increasing the range of the score. After further evaluation and research, the SVD score might be an easy way to detect patients at risk of future cognitive decline.

\footnotetext{
1 Clinical Implications. Hypertension. 2014;64:445-445.

2 Coca A, Sierra C. Beyond subjective cognitive failures in patients with hypertension? Hypertension. 2014;64:455-456. 3 https://www.gezondheidsnet.nl/hersenen-en-geheugen/geheugenklachten-voorspellen-microbloedingen-in-dehersenen
} 


\section{The role of hypertension in CSVD and cognition}

Until now, only few studies had investigated longitudinal relationships between MRI markers of CSVD and cognitive function or cognitive decline. In this thesis, we showed that progression of periventricular white matter hyperintensities over 4 year is associated with cognitive decline in patients with hypertension. In addition, we reported that progression is more important in predicting cognitive decline than periventricular white matter hyperintensities already present at the start of the study. These results implicate that it is important to prevent progression of cSVD.

This thesis emphasizes the social and economic impact of hypertension-related brain damage and cognitive deficits and the high need of investigating the mechanisms of CSVD to consequently find prevention or treatment targets. The awareness of the research society is increasing and recently, the European Union's Horizon 2020 research and innovation programme funded the SVDs@target project. This project aims to elucidate the different mechanisms that are involved in microvascular dysfunction in cSVD. Maastricht University participates in this large European study. An important part of the study focuses on blood pressure and aims to unfold the pathways by which blood pressure affects SVD or cognitive function and identify novel therapeutic mechanisms that target the microvascular function of brain vessels.

In addition to studies focusing on the mechanisms of SVD or cognitive deficits, studies investigating the effect of antihypertensive medication on development or progression of CSVD or cognitive problems in patients with hypertension are needed. Existing randomized clinical trials did not give conclusive evidence about the effects of antihypertensive medications in this regard. Therefore, well-performed randomized controlled trials are needed to improve prevention of cognitive decline in patients with hypertension.

\section{Conclusion}

The results described in this thesis could help to (more easily) identify patients at high risk of lower cognitive function, future cognitive decline or CSVD-related brain damage. This provides information for clinicians to determine which patients are in need of further investigation, such as an extensive neuropsychological assessment or brain imaging. First, we showed the importance of awareness for subjective cognitive failures in patients with hypertension and the need of asking for cognitive problems during consultation of patients with hypertension. We spread these results in scientific and non-scientific ways to increase understanding in both patients and clinicians. Second, we showed that a recently proposed total SVD score could be useful in predicting cognitive decline. This score provides a more complete overview instead of studying individual MRI markers and can be easily applied in clinical practice. After further investigation this score could be used to identify 
patients at high risk of cognitive decline. Third, we showed that progression of cSVD could result in a steeper cognitive decline in patients with hypertension. These results contribute to the increasing awareness of the role of blood pressure in CSVD and related cognitive problems. It emphasizes the importance of new studies in this field, such as the SVDs@ target project, to find new ways to prevent CSVD progression and, consequently, cognitive deficits. 


Dankwoord 

Na jaren werk is het zover! Die jaren zijn ontzettend leerzaam en leuk geweest. Daarvoor ben ik veel mensen dank verschuldigd.

Prof. dr. R.J. van Oostenbrugge, beste Robert. De stelling die jij opperde "The less professors lecture, the more students may learn", zou in het proefschrift van ieder van jouw promovendi kunnen staan. Ik heb wel even moeten wennen aan jouw snelle manier van communiceren, je gewoonte af en toe weg te lopen midden in een zin of een reactie van maar drie woorden terug te krijgen op een uitgebreide mail. Met één kritische vraag kon jij het probleem aanwijzen waar ik tegenaan liep en wist je me uit te dagen zelf met een oplossing te komen. Op die manier heb ik de afgelopen jaren veel meer geleerd dan als je me altijd het makkelijke antwoord had gegeven waar ik op dat moment op hoopte. Veel dank daarvoor!

Dr. J. Staals, beste Julie. Ondanks je drukke agenda vond jij altijd snel tijd om naar mijn manuscripten te kijken, even iets te overleggen of samen ergens over te brainstormen. Dank je wel dat je ieder stuk dat ik je stuurde grondig en kritisch las, ieder antwoord perfect doordacht en, niet te vergeten, elke MRI scan precies beoordeelde. Je hebt me geduldig alle uitleg gegeven over de vasculaire neurologie en over het onderzoek dat ik vroeg. Al snel ontdekte ik voor welke vragen ik beste bij wie terecht kon. Samen met Robert vormde je het perfecte promotieteam.

Beste Marjolein. Zonder jou was dit boekje er niet geweest. Dank je wel voor het opzetten van de onderzoeken, het verzamelen van de baseline data en alle hulp bij het vervolg ervan.

Beste Prof. dr. Peter de Leeuw en Prof. dr. Bram Kroon. Bedankt voor het uitvoeren van het eerste HYBRiD onderzoek, voor het meedenken over de onderzoeksvragen en de kritische blik op mijn manuscripten.

Beste Annelien. Ook jij bent betrokken geweest bij de opzet van de onderzoeken. Helaas hebben we niet zoveel samen kunnen werken. Bedankt dat je altijd beschikbaar bent gebleven voor vragen. Wie weet komen we in de toekomst allebei weer terug bij het neurovasculaire onderzoek.

Beste Chantal en Jacqueline. Alweer 5 jaar geleden begon ik aan mijn klinische stage bij jullie op de afdeling Medische Psychologie. Jullie leerden me de belangrijkste klinische vaardigheden op het gebied van anamneses en neuropsychologische onderzoeken. Zonder jullie had ik dit promotieonderzoek niet kunnen uitvoeren. Wat hebben jullie me veel geleerd in zes maanden tijd. Bedankt daarvoor. 
Beste collega's van Adelante. Dank jullie wel dat jullie me wegwijs hebben gemaakt in de wereld van mensen met tinnitus en met andere gehoorproblemen. Ik heb een erg leuk en leerzaam jaar gehad bij jullie.

Beste Syenna en Elles. Jullie zaten een stukje verder weg in het ziekenhuis, maar wat fijn dat jullie mijn "neuro-vasculaire-psychologie" collega's waren. Syenna, zonder jou had ik het congres in Tokyo waarschijnlijk aan me voorbij laten gaan. Bedankt dat je me mee op sleeptouw hebt genomen. Elles, samen hebben we twee leuke congressen om op terug te kijken. Dank voor de gezellige avonden in Amsterdam en Praag.

Beste onderzoekscollega's, in het bijzonder lieve Neuronols. We hebben regelmatig geklaagd dat het wel erg druk werd op onze kamer, maar stiekem was het ook heel gezellig. Natuurlijk ga ik de tosti-middagen, de koffie- of fruitmomentjes en de neuro-weekendjes missen. Maar vooral de goede sfeer op onze kamer, waar altijd wel iemand was om even mee te kletsen, te discussiëren, frustraties te delen of te lachen. Dank jullie wel voor de leuke jaren.

Lieve Eleana. Bijna tegelijk begonnen we aan ons onderzoek en wat ben ik blij dat ik mijn promotietraject met jou hier naast me kan afsluiten. Dank voor je gezelligheid en je altijd aanwezige belangstelling. Ik hoop dat we samen nog heel vaak van een koffie, brownie of cheesecake kunnen genieten.

Lieve Daniëlle. Wie had gedacht dat wij niet alleen vriendinnen, klasgenootjes, huisgenootjes, maar ook nog collega's zouden worden? Het zal wennen worden je minder te zien na al die tijd samen. Dank je wel dat je altijd voor me klaar staat en in bent om samen iets gezelligs te doen.

Lieve Charida, Daniëlle, Désirée, Maud, Monique, Nathalie, Nickie en Pascalle. Na de gezellige jaren op de middelbare school hadden anderen niet verwacht dat onze vriendschap na het eindexamen nog lang zou duren. Maar wij wisten wel beter en dat hebben we laten zien. Ook al zien we elkaar nu wat minder, een avondje met z'n allen zal altijd ouderwets gezellig blijven. Dank jullie wel daarvoor! Pas en Maud, het liefst had ik jullie vandaag hier ook naast me gehad. Dank jullie wel dat ik altijd op jullie kan rekenen!

Lieve Anouk, Jorik, Jonas en Naomi. Helaas heeft de bende van Belfort niet zo lang bestaan, maar gelukkig wonen we nog steeds vlak bij elkaar en is er tijd voor etentjes en dagjes weg. Dank jullie wel voor alle gezelligheid en dat ik dankzij jullie trotse tante ben geworden van Nora, Lune, Suze en Seppe. Er is geen betere manier om werkgepieker te vergeten dan tijd door te brengen met jullie boefjes; hun vrolijkheid en onbezorgdheid is aanstekelijk. 
Lieve papa en mama. Dank jullie wel voor het fijne thuis dat jullie nog altijd geven. Jullie hebben ons drieën altijd de vrijheid gegeven om onze eigen keuzes te maken. Ondanks dat, of misschien juist daardoor, ben ik uiteindelijk in jullie wetenschappelijke voetsporen getreden. Hopelijk zitten jullie vandaag net zo trots op de eerste rij als ik 4 jaar geleden op deze plek naast mama stond.

Lieve Roel. Wat is het fijn als er altijd iemand is die het volle vertrouwen in je heeft. Als ik het gevoel heb dat iets me niet gaat lukken, ben jij er om dit tegen te spreken. Maar alleen al je aanwezigheid zorgt voor alle steun die ik nodig heb. Dank je wel voor alles! 

Curriculum vitae 

Renske Uiterwijk werd geboren op 15 augustus 1990 in Maastricht. In 2008 behaalde zij haar VWO-diploma aan het Bonnefanten College. In dat jaar startte Renske met de studie Psychologie aan de Universiteit Maastricht en in 2011 ontving zij haar bachelor diploma. Een jaar later slaagde zij cum laude voor de master Neuropsychology, tevens aan de Universiteit Maastricht. Na haar master deed zij een klinische stage bij de afdeling Medische Psychologie van het Maastricht Universitair Medisch Centrum (MUMC+). In mei 2013 startte Renske haar promotietraject bij de afdeling Neurologie van het MUMC+. Vanaf oktober 2016 combineerde zij haar onderzoek met een functie als psycholoog bij de afdeling Audiologie \& Communicatie van Adelante Zorggroep te Blerick-Venlo. Vanaf november 2017 zal ze werkzaam zijn bij U-center als psycholoog.

Renske Uiterwijk was born in Maastricht on 15 August 1990. In 2008 she graduated from secondary school at Bonnefanten College. In the same year Renske started studying Psychology at Maastricht University and in 2011 she received her bachelor degree. One year later, she graduated cum laude for the master Neuropsychology, also at Maastricht University. After her master she performed a clinical internship at the department of Medical Psychology at Maastricht University Medical Centre (MUMC+). In May 2013 Renske started working as a PhD student at the department of Neurology at MUMC+. Since October 2016 she combined her research with a job as psychologist at the department of Audiology \& Communication at Adelante Zorggroep in Blerick-Venlo. As of November 2017, she will be working as a psychologist at U-center.

\section{List of publications}

Uiterwijk R, Huijts M, Staals J, Duits A, Gronenschild E, Kroon AA, de Leeuw PW, van Oostenbrugge RJ. Subjective cognitive failures in patients with hypertension are related to cognitive performance and cerebral microbleeds. Hypertension, 2014; 64: 653-657.

Uiterwijk R, Huijts M, Staals J, Rouhl RPW, de Leeuw PW, Kroon AA, van Oostenbrugge RJ. Endothelial activation is associated with cognitive performance in patients with hypertension. American Journal of Hypertension, 2016; 29: 464-469.

Uiterwijk R, van Oostenbrugge RJ, Huijts M, de Leeuw PW, Kroon AA, Staals J. Total cerebral small vessel disease MRI score is associated with cognitive decline in executive function in patients with hypertension. Frontiers in Aging Neuroscience, 2016; 8: 301. 
Uiterwijk R, Staals J, Huijts M, de Leeuw PW, Kroon AA, van Oostenbrugge RJ. MRI progression of cerebral small vessel disease and cognitive decline in patients with hypertension. Journal of Hypertension, 2017; 35: 1263-1270.

Zhang CE, Wong SM, Uiterwijk R, Staals J, Backes WH, Hoff El, Schreuder T, Jeukens CR, Jansen JF, van Oostenbrugge RJ. Intravoxel incoherent motion imaging in small vessel disease: Microstructural integrity and microvascular perfusion related to cognition. Stroke, 2017; 48: 658-663.

Uiterwijk R, Staals J, Huijts M, de Leeuw PW, Kroon AA, van Oostenbrugge RJ. Framingham stroke risk profile is related to cerebral small vessel disease progression and lower cognitive performance in patients with hypertension. Accepted for publication in The Journal of Clinical Hypertension.

Uiterwijk R, Staals J, Huijts M, van Kuijk SMJ, de Leeuw PW, Kroon AA, van Oostenbrugge RJ. Hypertensive organ damage predicts future cognitive performance: A 9-year follow-up study in patients with hypertension. Submitted.

Zhang CE, Wong SM, Uiterwijk R, Backes WH, Jansen JF, Jeukens CR, van Oostenbrugge RJ, Staals J. Blood-brain barrier leakage in relation to white matter hyperintensity volume and cognition in small vessel disease and normal ageing. Submitted. 